\title{
Impact of Incident Cancer on Coronary Artery Disease-Related Concomitant Medication Adherence, Short-Term Health and Economic Outcomes Among Elderly Medicare Beneficiaries with Coronary Artery Diseas
}

Ishveen Kaur Chopra

Follow this and additional works at: https://researchrepository.wvu.edu/etd

\section{Recommended Citation}

Chopra, Ishveen Kaur, "Impact of Incident Cancer on Coronary Artery Disease-Related Concomitant Medication Adherence, Short-Term Health and Economic Outcomes Among Elderly Medicare Beneficiaries with Coronary Artery Diseas" (2017). Graduate Theses, Dissertations, and Problem Reports. 5362.

https://researchrepository.wvu.edu/etd/5362

This Dissertation is protected by copyright and/or related rights. It has been brought to you by the The Research Repository @ WVU with permission from the rights-holder(s). You are free to use this Dissertation in any way that is permitted by the copyright and related rights legislation that applies to your use. For other uses you must obtain permission from the rights-holder(s) directly, unless additional rights are indicated by a Creative Commons license in the record and/ or on the work itself. This Dissertation has been accepted for inclusion in WVU Graduate Theses, Dissertations, and Problem Reports collection by an authorized administrator of The Research Repository @ WVU.

For more information, please contact researchrepository@mail.wvu.edu. 


\title{
IMPACT OF INCIDENT CANCER ON CORONARY ARTERY DISEASE-RELATED
} CONCOMITANT MEDICATION ADHERENCE, SHORT-TERM HEALTH AND ECONOMIC OUTCOMES AMONG ELDERLY MEDICARE BENEFICIARIES WITH CORONARY ARTERY DISEASE

\author{
Ishveen Kaur Chopra \\ Dissertation submitted to the School of Pharmacy \\ at West Virginia University \\ in partial fulfillment of the requirements \\ for the degree of \\ Doctor of Philosophy \\ in \\ Health Services and Outcomes Research
}

Usha Sambamoorthi, Ph.D., Chair Xi Tan, Ph.D.

Nilanjana Dwibedi, Ph.D. Malcolm D Mattes, MD

Patricia Findley, DrPH, MSW, LCSW

Department of Pharmaceutical Systems and Policy

Morgantown, West Virginia

2017

Keywords: Cancer, Coronary artery disease, Concomitant medication adherence, Health outcomes, Health expenditures

Copyright 2017 Ishveen Kaur Chopra 


\begin{abstract}
Impact of Incident Cancer on Coronary Artery Disease-Related Concomitant Medication Adherence, Short-Term Health and Economic Outcomes among Elderly Medicare Beneficiaries with Coronary Artery

Disease
\end{abstract}

\title{
Ishveen Kaur Chopra
}

Coronary artery disease (CAD) is one of the most burdensome chronic conditions in the elderly. The two key goals of long-term management of CAD are (i) to reduce symptoms and ischemia and (ii) prevent myocardial infarction and death, by lowering lipids and blood pressure. Of all the risk reduction strategies, use and adherence to concomitant pharmacotherapy with statins and beta-blockers, angiotensin-converting enzyme inhibitors (ACEIs) or angiotensin II receptor blockers (ARBs) have been shown to be highly effective and has become the cornerstone of CAD management. However, adherence to concomitant pharmacotherapy can be influenced by many factors including the development of other life-threatening conditions such as cancer. To date, no real-world study has assessed how incident cancer can affect adherence to concomitant pharmacotherapy and whether concomitant pharmacotherapy can minimize the negative effects of some cancer treatments on CAD-related morbidity and expenditures among individuals with CAD and incident cancer. To fill the knowledge gap, the three related aims of this dissertation were to analyze: 1) the association between incident breast, colorectal, and prostate cancer diagnosis and adherence to statins and/or ACEIs/ARBs/beta-blockers among elderly fee-for-service (FFS) Medicare beneficiaries with pre-existing CAD; 2) the impact of non-adherence to these medication classes on short-term CAD-related hospitalizations in patients with incident cancer diagnosis; and 3) the impact of incident cancer diagnosis on short-term CAD-related inpatient and outpatient healthcare expenditures. The study used a retrospective observational longitudinal cohort study design was conducted among elderly Medicare FFS beneficiaries with pre-existing CAD and those with incident breast cancer (BC), colorectal cancer (CC), or prostate cancer (PC), using multiple years (2005-2012). The study data was derived from the cancer registry data from Surveillance, Epidemiology and End Results (SEER) program linked with the Medicare claims data, the American community survey census-tract files and Area Health Resource Files. Aim 1 and Aim 3 also included 5\% non-cancer random sample of Medicare beneficiaries, residing in SEER regions, with pre-existing CAD. Each individual was observed for 48 months with 24-month baseline (for identification of CAD and baseline characteristics) period, 12month pre-index, and 12-month post-index periods. In the first aim, only $28.9 \%$ of the elderly with CAD were adherent to both statins and ACEIs/ARBs/beta-blockers. In the adjusted analyses, women $[\mathrm{AOR}=0.70 ; 95 \% \mathrm{CI}=$ $0.58,0.81 ; P<0.0001]$ and men [AOR $=0.63 ; 95 \% \mathrm{CI}=0.51,0.75 ; P<0.0001]$ with $\mathrm{CC}$ and men with PC [AOR $=$ $0.92 ; 95 \% \mathrm{CI}=0.85,0.99 ; P=0.022]$ were significantly less likely to be adherent to both medication classes compared to women and men with NC, respectively. No significant differences in adherence to medications were observed among women with BC compared to women with NC. Even among those using single medication class, women $[\mathrm{AOR}=0.64 ; 95 \% \mathrm{CI}=0.50,0.79 ; P<0.0001]$ and men with $\mathrm{CC}[\mathrm{AOR}=0.59 ; 95 \% \mathrm{CI}=0.42,0.76 ; P<$ 0.0001] were significantly less likely to be adherent to that medication class compared to women and men with NC. In the second aim, adherence to both statins and ACEIs/ARBs/beta-blockers was estimated at $31.2 \%$ during the 120day period immediately after cancer diagnosis; $13.7 \%$ were not adherent to both medication classes during the same period; $27.4 \%$ had CAD-related hospitalizations immediately after cancer diagnosis and this percentage declined to $10.6 \%$ during the last four months of the post-cancer period. In the adjusted analyses, those not adherent to both statins and ACEIs/ARBs/beta-blockers were more likely to have CAD-related hospitalization compared to those who were adherent to both medication classes [AOR $=1.82 ; 95 \% \mathrm{CI}=1.72,1.92 ; P<0.0001$ ]. In the third aim, overall, CAD-related mean healthcare expenditures at pre-index period accounted for about $32.6 \%-39.5 \%$ of total expenditures among women and $41.5 \%-46.8 \%$ among men. In the adjusted GLMM, all cancer groups had significantly higher CAD-related healthcare expenditures compared to the non-cancer groups. Women with CC $153 \%$ higher expenditures compared to women with no cancer $[\beta=0.93, \mathrm{P}<0.0001]$. Men with CC had $166 \%$ higher expenditures compared to men with NC [ $\beta=0.98, \mathrm{P}<0.0001]$. Further, men and women with CC had 57\% and 55\% higher expenditures compared to men with PC and women with BC, respectively. In summary, the study findings, collectively, suggest that cancer diagnosis negatively impacts adherence to CAD pharmacotherapy. Reduction in adherence was associated with increase in CAD-related hospitalizations and subsequent increase in CAD-related expenditures. This warrants the integration of cardiovascular care in the elderly diagnosed with cancer. 
Future studies need to explore whether the emerging collaborative care models, such as medical homes, can reduce inpatient use, and consequently, CAD-related expenditures. 


\section{Table of Contents}

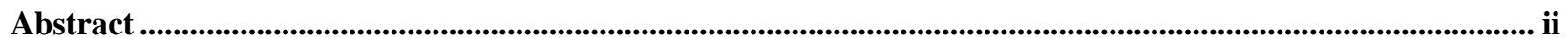

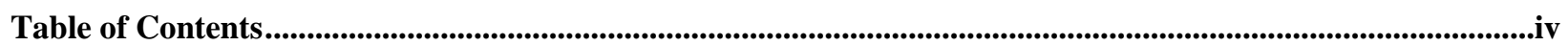

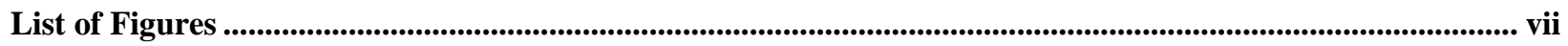

List of Tables...................................................................................................................................................................... viii

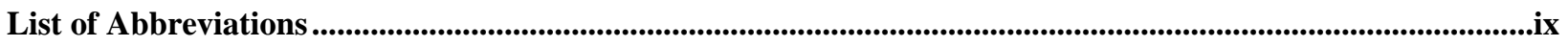

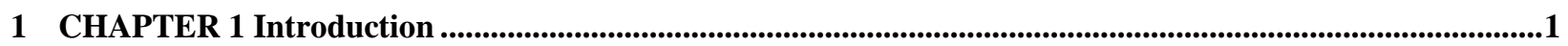

1.1 Coronary Artery Disease ..........................................................................................................................1

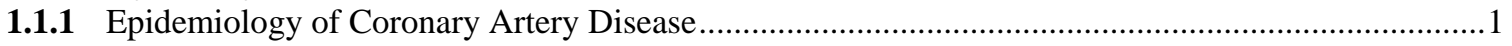

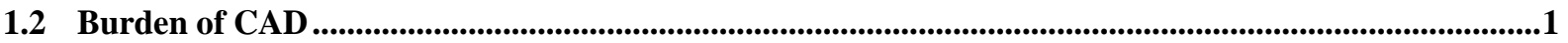

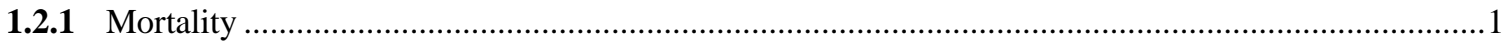

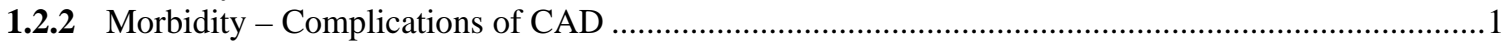

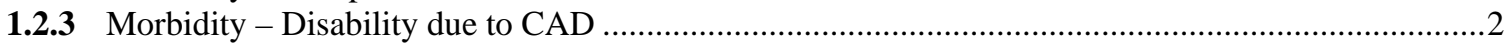

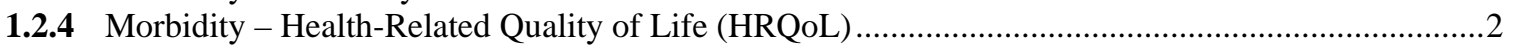

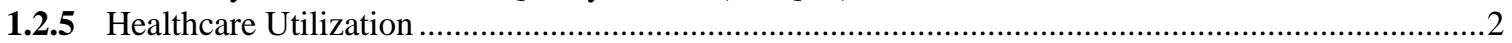

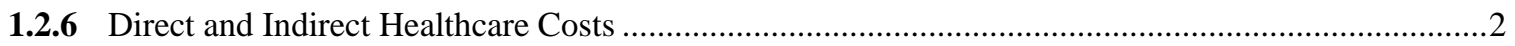

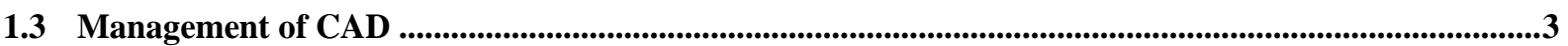

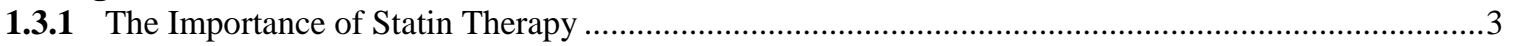

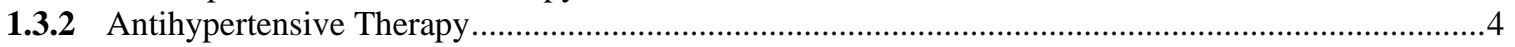

1.3.2.1 The Importance of Beta-blockers, ACEIs, and ARBs ............................................................

1.4 CAD Management and the Role of Incident Cancer....................................................................................4

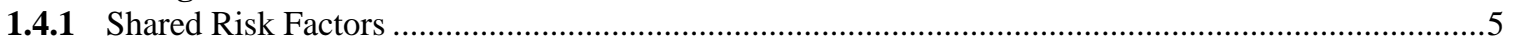

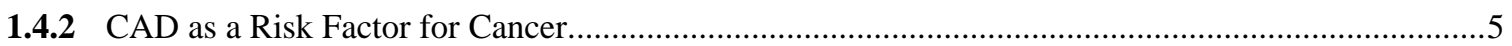

1.4.3 Negative Effects of Some Cancer Treatments on Cardiovascular Health.............................................5

1.4.4 Dominant Condition as the Driver of Treatments: CAD or Cancer? .................................................6

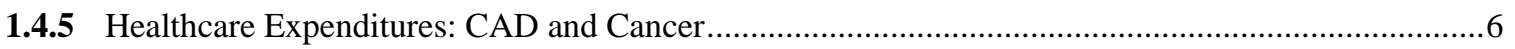

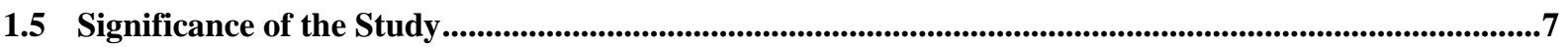

1.5.1 Burden of CAD in Comparison to Other Heart Diseases.........................................................

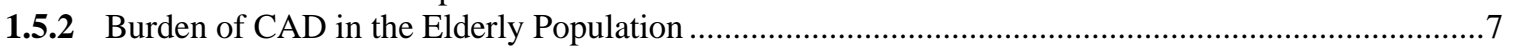

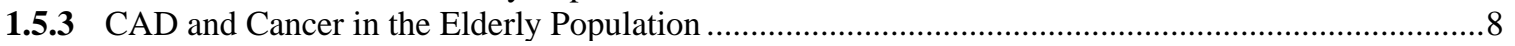

1.5.4 Statins, Beta-blockers, ACEIs, and ARBs and CAD-related Outcomes .............................................

1.5.5 Cost Implications of CAD and Cancer in the Elderly Population.......................................................

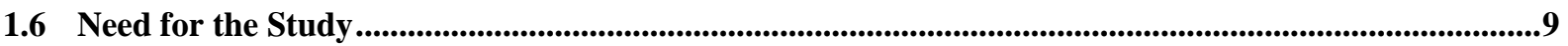

1.6.1 Medicare as the Primary Payer of CAD and/or Cancer ......................................................................

1.6.2 Disproportionate Burden of CAD among Elderly Population ...........................................................9

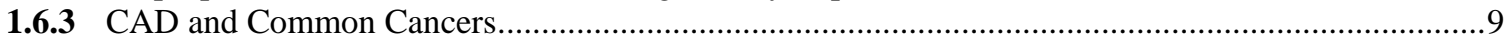

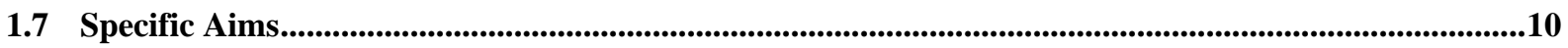

1.8 Innovation and Unique Contribution of the Study ..........................................................................11

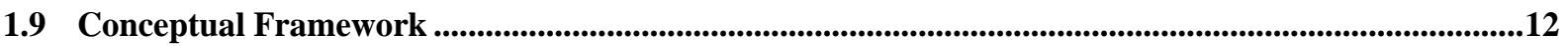

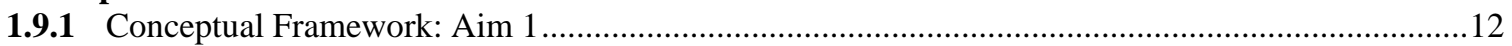

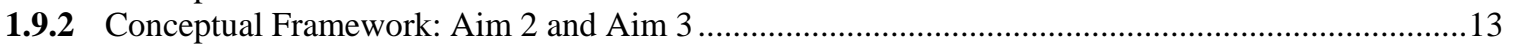

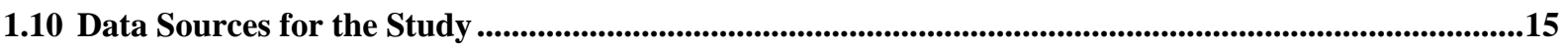

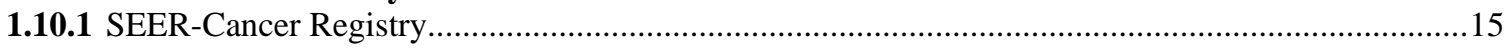

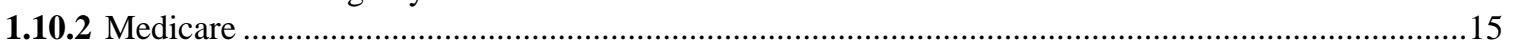

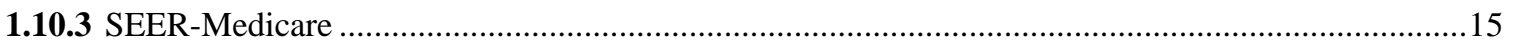

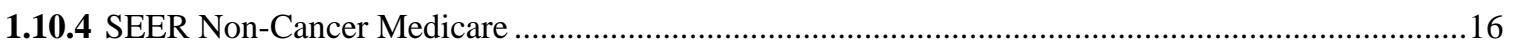

1.10.5 American Community Survey (ACS) Census Tract Files..............................................................16

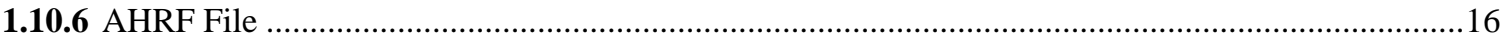

1.10.7 County Health Ranking File .......................................................................................................16 
2 CHAPTER 2 Incident Cancer and Adherence to Statins and Antihypertensive Medications for Coronary Artery Disease among Elderly Medicare Beneficiaries ..............................................................................................17

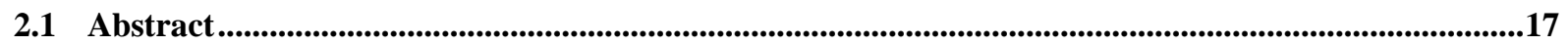

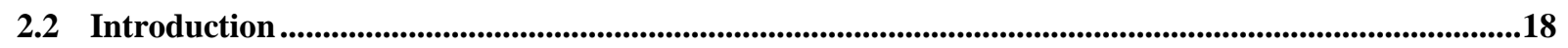

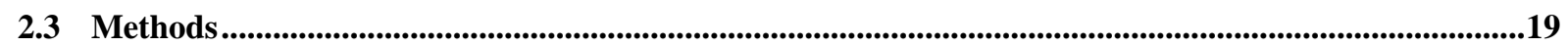

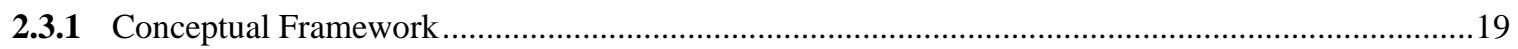

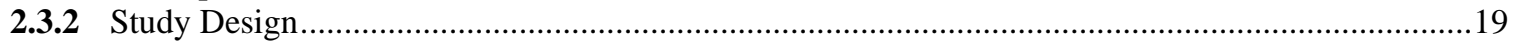

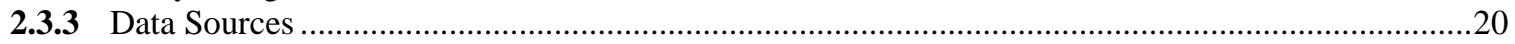

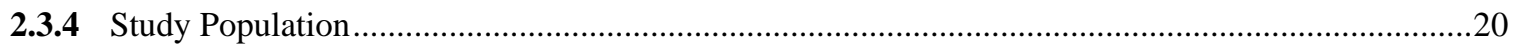

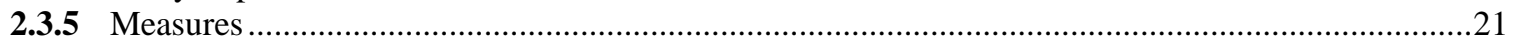

2.3.5.1 Dependent Variable: Adherence to Statins, ACEIs/ARB/Beta-blockers................................21

2.3.5.2 Key Independent Variable: Cancer Type and Sex ................................................................21

2.3.5.3 Other Independent Variables .................................................................................................23

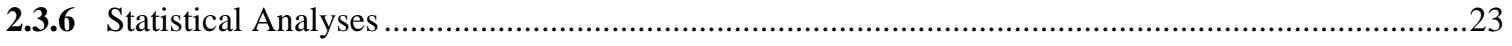

2.3.6.1 Inverse Probability Treatment Weights (IPTW) .....................................................................24

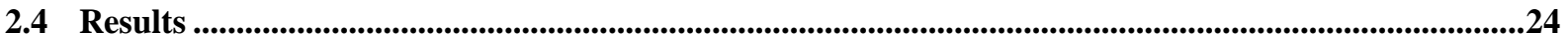

2.4.1 Characteristics of the Study Population Before and After IPTW Adjustment ..................................24

2.4.2 Unadjusted and Adjusted Relationship between Cancer and Adherence to Statins and/or

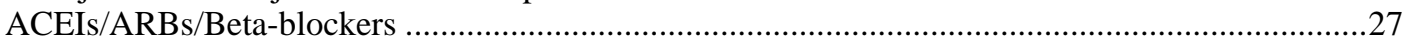

2.4.2.1 Cancer versus No Cancer and Medication Adherence...........................................................27

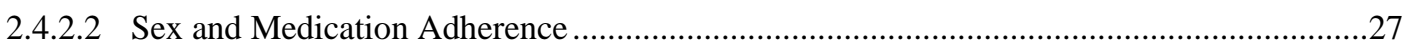

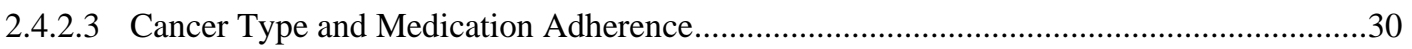

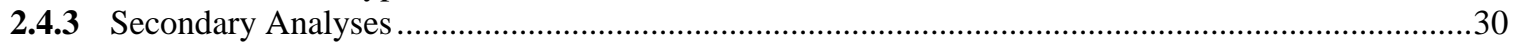

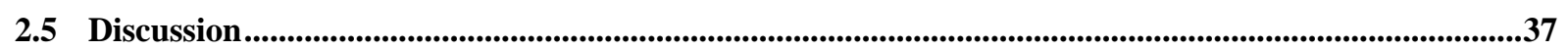

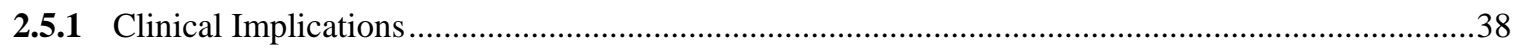

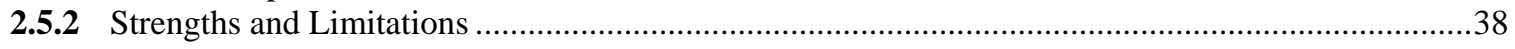

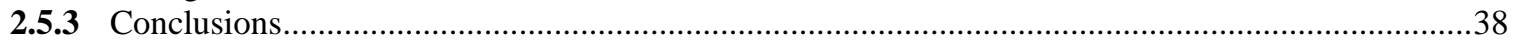

3 CHAPTER 3 Non-adherence to Statins and Antihypertensive Medications and Hospitalizations among Elderly Fee-for-Service Medicare Beneficiaries with Pre-existing Coronary Artery Disease and Incident Cancer.

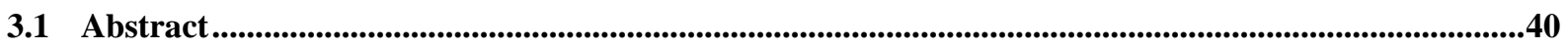

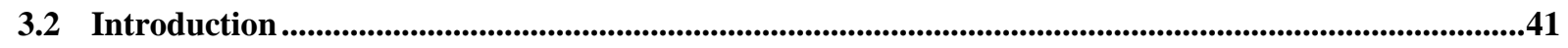

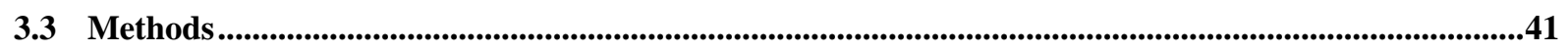

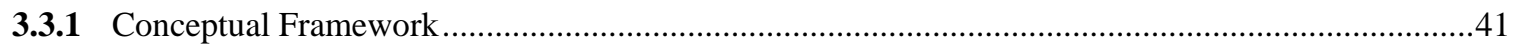

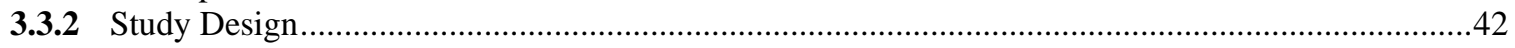

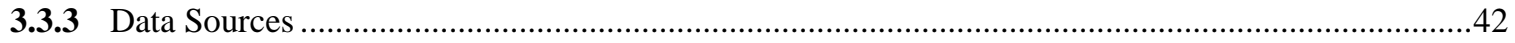

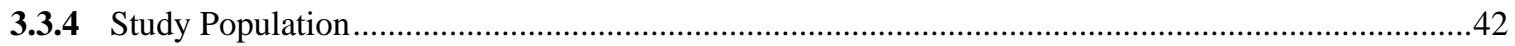

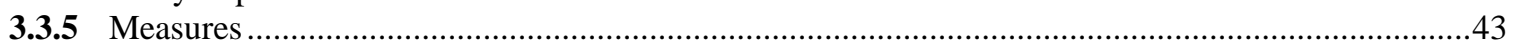

3.3.5.1 Dependent Variable: Any Hospitalization for CAD Events ...................................................43

3.3.5.2 Key Independent Variable: Adherence to Statins, ACEIs/ARB/Beta-blockers.....................43

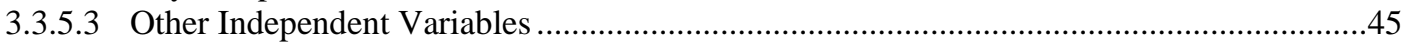

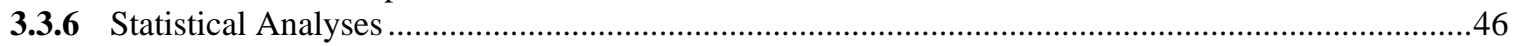

3.3.6.1 Inverse Probability Treatment Weight (IPTW) .......................................................................46

3.3.6.2 Instrument Variable (IV) Regression..................................................................................

3.4 Results ............................................................................................................................................................51

3.4.1 Characteristics of the Study Population …………..............................................................................51

3.4.1.1 Characteristics of the Study Population by Medication Adherence Categories - Before and After IPTW Adjustment .................................................................................................51

3.4.1.2 Unadjusted and Adjusted Associations between Adherence to Statins and/or ACEIs/ARBs/Beta-blockers and CAD-related Hospitalization.............................................57

3.4.2 Sensitivity Analyses...................................................................................................................6

3.4.3 Adjusted Associations between Other Independent Variables and CAD Hospitalization .................60

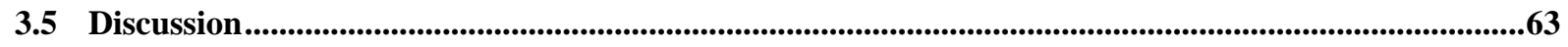




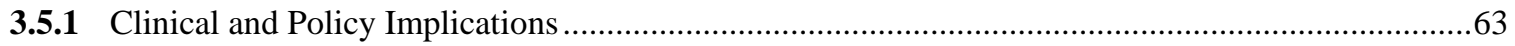

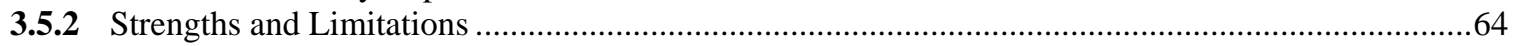

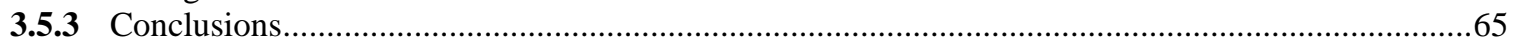

4 CHAPTER 4 Impact of Incident Cancer on Short-term CAD-related Medicare Expenditures among Medicare Beneficiaries with Pre-existing Coronary Artery Disease ...................................................................66

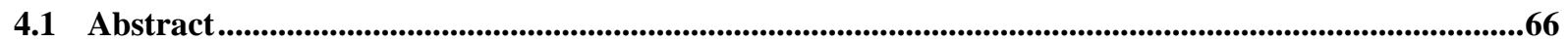

4.2 Introduction ..........................................................................................................................................................67

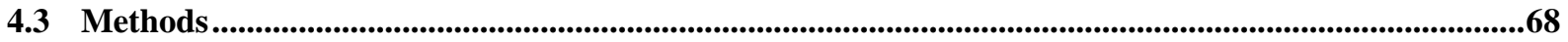

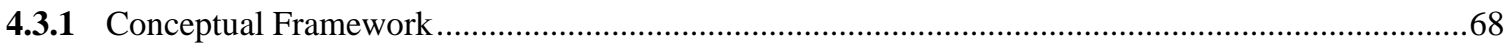

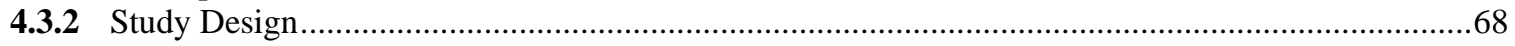

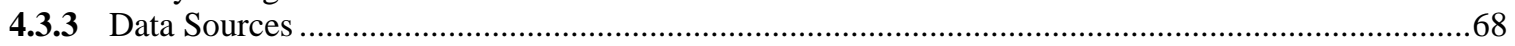

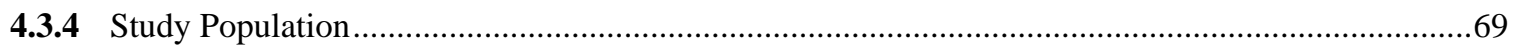

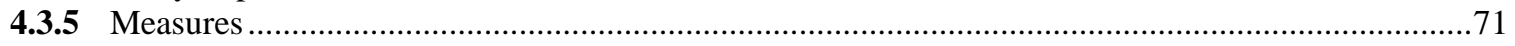

4.3.5.1 Dependent variable: CAD-related Expenditures …................................................................71

4.3.5.2 Key independent Variable - Cancer Type and Sex .............................................................71

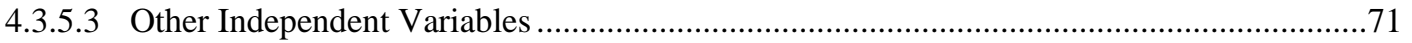

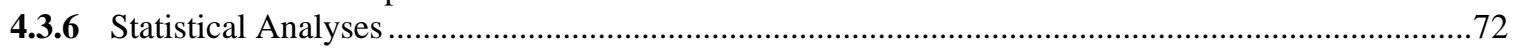

4.4 Results ...............................................................................................................................................................78

4.4.1 Characteristics of the Study Population before and after IPTW Adjustment.....................................78

4.4.2 CAD-related Expenditures over time by Cancer and Non-Cancer Status...........................................80

4.4.3 Adjusted Relationships between Cancer and CAD-related Healthcare Expenditures..........................80

4.4.3.1 Cancer versus No cancer and CAD-related Healthcare Expenditures .....................................80

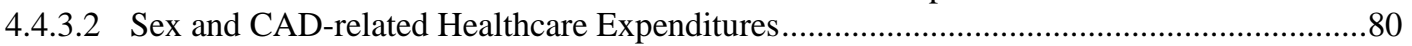

4.4.3.3 Cancer Type and CAD-related Healthcare Expenditures ......................................................80

4.4.4 Relationship between Other Independent Variables and Expenditures................................................87

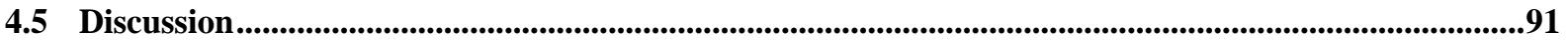

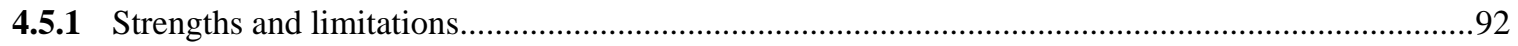

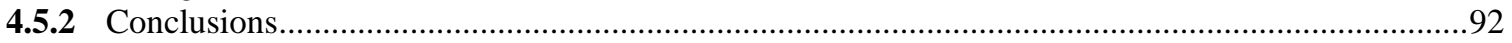

5 Chapter 5 Discussion of Findings and Research Implications ..................................................................94

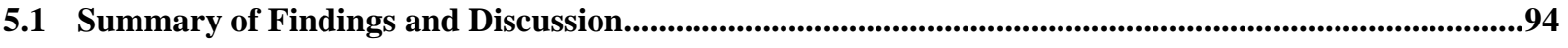

5.2 Clinical Implications of the Findings ........................................................................................................96

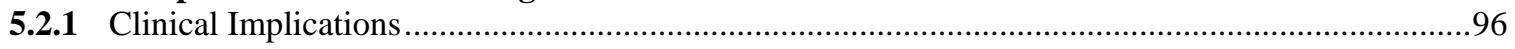

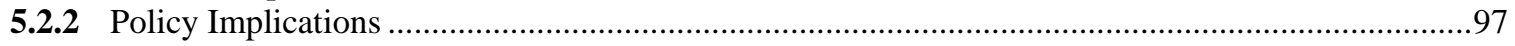

5.3 Unique Contributions of the Study ........................................................................................................98

5.4 Strengths and Limitations of the Study .........................................................................................98

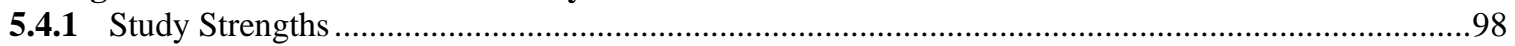

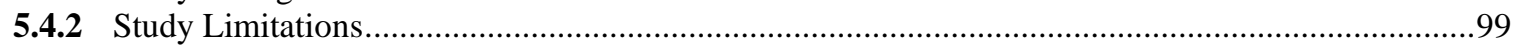

5.5 Suggestions for Future Research .................................................................................................................99

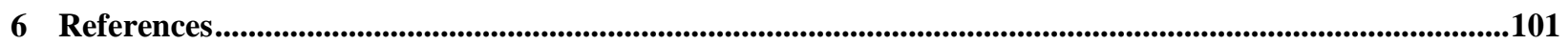

Appendix A. Codes related to cancer diagnosis and treatment ...................................................................................114

Appendix B. List of statins.............................................................................................................................115

Appendix C. List of angiotensin-converting enzyme inhibitors.................................................................................116

Appendix D. List of angiotensin II receptor blockers.....................................................................................................117

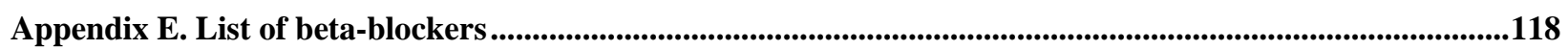

Appendix F. Heart disease ICD-9-CM diagnosis and procedure codes .................................................................119 


\section{List of Figures}

Figure 1.1 Conceptual framework adapted from Park and Jones for medication adherence.

Figure 1.2 Conceptual framework adapted from the Andersen Behavioral Model of Health Service Use for medication adherence and health outcomes.

Figure 1.3 Conceptual framework adapted from the Andersen Behavioral Model of Health Service Use for medication adherence and economic outcome.

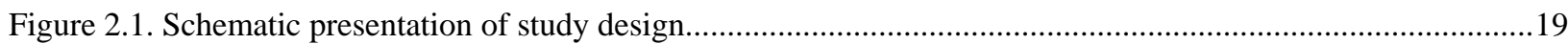

Figure 2.2. Schematic presentation of selection criteria for study cohort..........................................................22

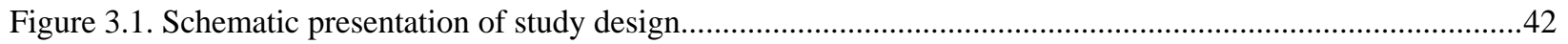

Figure 3.2. Schematic presentation of selection criteria for study cohort......................................................44

Figure 4.1. Schematic presentation of selection criteria for study cohort.....................................................70

Figure 4.2. Weighted Average CAD-related Expenditures among Cancer and Non-cancer Cohorts across 120-day

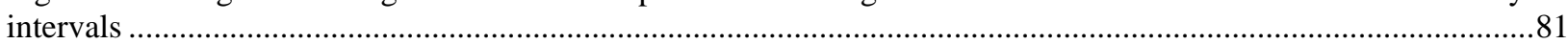




\section{List of Tables}

Table 2.1. Logistic Regression Models used to derive IPTW-Cancer and 5\% Non-Cancer Sample .........................25

Table 2.2. Number, Percent, and Weighted Percent of Patient-level Characteristics by Presence of Cancer..............26

Table 2.3. Weighted Percent and Unadjusted GEE Models with IPTW on Medication Adherence Categories .........28

Table 2.4. Adjusted GEE Models with IPTW on Adherence to Medication Adherence Categories..........................31

Table 2.5. Adjusted GEE Models with IPTW on Medication Adherence Categories-Other factors included based on conceptual framework

Table 2.6. Adjusted GEE models on Medication Adherence Categories among Patients with Colorectal Cancer $(\mathrm{N}=$ 3,104)

Table 3.1. Multinomial Logistic Regression Models used to derive IPTW ......................................................48

Table 3.2. Selected Characteristics of Study Population ................................................................................52

Table 3.3. Unweighted and Weighted Percent of Selected Characteristics by Adherence to Statins and/or

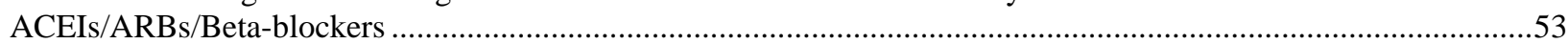

Table 3.4. Weighted percentage with CAD-related hospitalizations over different time periods. . .56

Table 3.5. Odds Ratios, Adjusted Odds Ratios, and 95\% Confidence Intervals of Medication Adherence Categories from Multinomial Logistic Regression Models with Repeated Measures on Hospitalization Categories - IPTW (Reference Group = No Hospitalizations)

Table 3.6. Sensitivity analysis: CAD Hospitalization Identified from Primary Diagnosis Odds Ratios, Adjusted Odds Ratios, and 95\% Confidence Intervals of Medication Adherence Categories GEE Models with IPTW on CAD Hospitalization. . .61

Table 4.1. Unweighted and Weighted Percent of Selected Characteristics by Cancer Diagnosis .............................74

Table 4.2. Multinomial Logistic Regression Models used to derive IPTW .......................................................76

Table 4.3. Mean and Standard Deviation of Coronary Artery Disease-related Expenditures by Cancer and NonCancer Adjusted with IPTW.

Table 4.4. Parameter Estimates from Generalized Linear Mixed Models on CAD-related Expenditures with IPTW 83 Table 4.5. Parameter Estimates from Generalized Linear Mixed Models on CAD-related Expenditures with IPTW 85 Table 4.6. Adjusted Relationship between Other Characteristics and CAD-related Expenditures, Repeated Measures Regressions with IPTW 


\section{List of Abbreviations}

ACEI

ADT

AHRF

AHRQ

AOR

ARB

AJCC

ACS

BCW

BP

BRFSS

CAD

CCM

CCW

CDC

CPT-4

DALY

DME

FFS

GEE

GLMM

HHA

HCPCS

HCUP

HPSA

HRQoL

ICD-9-CM

ICD-O-3

LDL-C

MACRA

MCC

MEDPAR

MI

MPR

$\mathrm{NCH}$

NCM
Angiotensin-converting enzyme inhibitors

Androgen deprivation therapy

Area Health Resources File

Agency for Healthcare Research and Quality

Adjusted odds ratio

Angiotensin II receptor blockers

American Joint Committee on Cancer

American Community Survey

Women with breast cancer

Blood pressure

Behavioral Risk Factor Surveillance System

Coronary artery disease

Men with colorectal cancer

Women with colorectal cancer

Centers for Disease Control and Prevention

Common Procedural Terminology, 4th Edition

Disability-adjusted life years

Durable Medical Equipment

Fee-for-service

Generalized estimating equations

Generalized linear mixed models

Home Health Agency

Health Care Procedure Classification Code

Healthcare Cost and Utilization Project

Health professional shortage area

Health-related quality of life

International Classification of Diseases, Ninth Revision, Clinical Modification

International Classification of Diseases for Oncology, 3rd Edition

Low-density lipoprotein cholesterol

Medicare Access and Children's Health Insurance Program Reauthorization Act

Multiple Chronic Conditions

Medicare Provider Analysis and Review

Myocardial infarction

Medication Possession Ratio

National Claims History

Men with no cancer 


$\begin{array}{ll}\text { NCW } & \text { Women with no cancer } \\ \text { NDC } & \text { National Drug Code } \\ \text { NHANES } & \text { National Health and Nutrition Examination Survey } \\ \text { OR } & \text { Odds ratio } \\ \text { PCM } & \text { Men with prostate cancer } \\ \text { PCP } & \text { Primary care physician } \\ \text { PDC } & \text { Proportion of days covered } \\ \text { PDE } & \text { Part D Event } \\ \text { PEDSF } & \text { Patient Entitlement and Diagnosis Summary File } \\ \text { QALY } & \text { Quality-adjusted life year } \\ \text { RR } & \text { Relative risk } \\ \text { SNF } & \text { Skilled Nursing Facility } \\ \text { SEER } & \text { Surveillance, Epidemiology, and End Results } \\ \text { SUMDENOM } & \text { Summarized Denominator } \\ \text { US } & \text { United States } \\ \text { USD } & \text { United States dollar } \\ \text { Wt } & \text { Weighted }\end{array}$




\section{CHAPTER 1}

\section{Introduction}

\subsection{Coronary Artery Disease}

\subsubsection{Epidemiology of Coronary Artery Disease}

Coronary artery disease (CAD), also referred to as coronary heart disease or ischemic heart disease, is caused by atherosclerosis, is a disease in which there is narrowing or blockage of the coronary arteries, blood vessels that carry blood and oxygen to the heart [1]. Globally, CAD prevalence rate in 2006-2013 was 13.8\% [2]. The Framingham study investigators estimated a lifetime risk of developing CAD at $49 \%$ in men and 32\% in women [3].

In the United States (US), nearly 15.5 million adults aged 20 and older are diagnosed with CAD, [4] with prevalence rates of 6.2\% [4]. In 2015, approximately 635,000 new cases of CAD were diagnosed in adults aged 20 years and older [4]. Sex and race/ethnicity differences in CAD incidence and prevalence rates were also reported [46]. The average age-standardized CAD incidence rates have been reported to be 9.0 per 1,000 person years for black men versus 8.1 for white men and 5.0 for black women versus 3.4 for white women [5]. Hispanics (6.1\%) have higher age-adjusted prevalence of CAD as compared to whites (5.8\%) [6]. Women have lower prevalence of CAD (5.0\%) as compared to men (7.6\%) [4]. However, it is not known as to whether this lower prevalence is due to differences in presentation of CAD leading to under-detection of CAD in women $[7,8]$.

\subsection{Burden of CAD}

\subsubsection{Mortality}

Although the survival rate of CAD has been steadily improving [9], it still remains the leading cause of mortality worldwide; with 7.4 million deaths in 2012, 13.2\% of total global mortality [10]. It is projected that by 2020, worldwide, 11.1 million deaths will be due to CAD [11]. In the US, CAD is responsible for more than twothirds of all heart-related mortality, with more than 380,000 deaths annually [12]. CAD claims more lives than all forms of cancer combined [4]. Mortality from CAD disproportionately affects the elderly, men, and racial minorities $[4,13,14]$. For example, $80 \%$ of CAD-related mortality is reported among elderly population (65 years and older) [4].

\subsubsection{Morbidity - Complications of CAD}

Worldwide, CAD is the leading cause of morbidity, with 32.4 million myocardial infarctions (MI) every year $[15,16]$. In the US, approximately 1.5 million individuals experience MI each year, and amongst them, majority are either hospitalized for unstable angina or for treatment of stable chest pain syndromes. Every year, nearly 735,000 adults in the US have a heart attack, among which 525,000 are a first heart attack and 210,000 are recurrent heart attacks [12]. Observational studies on patients recovering from an acute MI reported that 1 in 5 patients had recurring angina within one year, even after intensive treatment [17]. 


\subsubsection{Morbidity - Disability due to CAD}

Globally, the overall burden of CAD (measured in terms of disability-adjusted life years (DALYs) is projected to increase to 82 million DALYs in 2020 from about 47 million in 1990 [18]. CAD is the leading cause of DALYs in the US for both males and females (11.2\% of the total) [19]. In the global burden of disease report, US was ranked 12 among 15 countries for age-standardized rates of DALYs for CAD relative to comparator countries (where 1 indicated best performance and 15 worst performance) [20].

\subsubsection{Morbidity - Health-Related Quality of Life (HRQoL)}

Another aspect of morbidity is the humanistic outcomes, specifically HRQoL. Individuals with CAD may experience poor HRQoL due to the symptom burden, complications resulting from CAD, such as MI, limited physical functioning capacity, and psychological stress [21]. It is reported that patients with MI have lower HRQoL compared to those with heart failure. Another study indicated that 25-30\% of individuals with cardiovascular event experience a constant feeling of uneasiness concerning their health, resulting in lower HRQoL [22]. Such lower HRQoL can lead to higher risk of mortality. A study by Bosworth et al (1999) observed 2,800 patients with CAD over period of 3.5 years. This study indicated that lower HRQoL was related to an approximately threefold greater all-cause risk of mortality and a 3.6-fold greater risk of mortality due to CAD [23].

\subsubsection{Healthcare Utilization}

The high burden of CAD can lead to intensive healthcare utilization. In the US, in 2010, there were 11 million ambulatory care visits, 10.5 million physician office visits, 587,000 emergency department visits, and 764,000 outpatient department visits with a primary diagnosis of CAD [4]. Further, Merrill et al (2008) indicated that 74.8\% of emergency department visits for CAD resulted in hospital admission [24]. Among the elderly Medicare beneficiaries ( $\geq 65$ years), of the average of 2.1 million hospitalizations per year, $42.0 \%$ of the visits occurred among those with CAD followed by heart failure (28.9\%), cardiac dysrhythmia (17.8\%), and other diseases of the heart (11.3\%) [25].

\subsubsection{Direct and Indirect Healthcare Costs}

The direct healthcare costs of caring for individuals with CAD are substantial; direct medical care costs of CAD in the US is projected at $\$ 106.4$ billion in 2030, a 198\% change from 2010 [26]. Nearly, 50\% of direct healthcare costs can be attributed to the hospitalizations [27]. Hospital or inpatient expenditures may be higher because of invasive procedures needed to manage CAD. According to the Healthcare Cost and Utilization Project (HCUP) statistics (2012), the mean hospital charges for a vascular/cardiac surgery or procedure was $\$ 78,897$, cardiac revascularization was $\$ 149,480$, and percutaneous interventions was $\$ 70,027$ [4].

The costs due to lost productivity, have been estimated to be $\$ 81$ billion [4, 27]. The indirect costs measured as lost productivity from morbidity and premature mortality can also be very high [4, 27, 28]. According to Heidenreich et al (2011), the indirect costs attributed to CAD is expected to increase to $\$ 112.3$ billion in 2030 from $\$ 73.2$ billion in 2010 [26]. 
In summary, CAD affects millions of adults in the US and contributes to substantial burden in terms of mortality, morbidity, poor humanistic outcomes, healthcare utilization, and costs of care.

\subsection{Management of CAD}

Long-term management of all forms of CAD follows the guidelines established for stable CAD [27]. According to clinical practitioners the management of CAD has two main goals: (i) to reduce symptoms and ischemia; (ii) to prevent MI and death [29]. There are many components to CAD management and they include physical activity, smoking cessation, blood pressure (BP) control, lipid control, diabetes, prescription of antiplatelet agents, betablockers, renin-angiotensin-aldosterone system blockers, nitrates, calcium antagonists, and ranolazine, and influenza vaccine [27].

Among all the risk reduction strategies, lowering BP and low-density lipoprotein cholesterol (LDL-C) levels are central to achieving the main goals of CAD management. A report from National Health and Nutrition Examination Survey (NHANES) indicated that nearly $44 \%$ decline in CAD mortality over time was attributable to changes in risk factors. Among all the risk factors, lower total cholesterol (24\%) and lower systolic BP (20\%) accounted for most of the decline in mortality compared to lower smoking prevalence (12\%) and decrease in physical inactivity (5\%) [4].

Reducing of LDL-C is associated with a reduced risk of recurring cardiovascular events in individuals with a diagnosis of CAD [30-34]. Clinical trials and prospective studies have shown that reducing blood pressure in patients with CAD can improve survival and prevent recurrent MI or stroke [35, 36]. Based on robust evidence, the Seventh Report of the Joint National Committee on Prevention, Detection, Evaluation, and Treatment of High Blood Pressure suggest lowering BP to 140/90 mmHg or less for patients with CAD [35]. Similarly, the Third Report of the National Cholesterol Education program recommends lowering LDL-C to less than $100 \mathrm{mg} / \mathrm{dL}$ in patients with CAD [37].

\subsubsection{The Importance of Statin Therapy}

For attaining guideline recommended LDL-C, pharmacotherapy that lowers lipid levels has become the standard of care for individuals with CAD. Although many types of lipid-lowering drugs (statins, bile acid sequestrants, fibrates, niacin, and ezetimibe) are available [37], statins are commonly prescribed for many reasons. The efficacy of statins in the primary and secondary prevention of cardiovascular events has been demonstrated by clinical trials [30-33]. For example, in the Cholesterol and Recurrent Events trial, pravastatin has been shown to reduce the risk of mortality, major coronary events, and the need for major invasive procedures compared to placebo [33].

In addition, statin therapy can be highly effective among older patients with CAD. Studies that have examined the effectiveness of statin therapy in reducing cardiovascular events and mortality in older patients suggest that older adults have greater reduction in coronary events as compared to younger subjects [38-40]. For example, among users of simvastatin, major coronary event rates were reduced by $32 \%$ in those $\geq 65$ years of age versus $19 \%$ in those $<65$ years of age [39]. The differential was even more striking in terms of mortality, which decreased by $45 \%$ 
in those $\geq 65$ years of age compared to $11 \%$ in patients $<65$ years of age [39]. Similar benefits among the elderly have been observed among Pravastatin users [38]. Statin therapy in the elderly has been projected to be cost-effective, where the incremental cost for quality-adjusted life year (QALY) in the elderly was reported to be $\$ 18,800$ much lower than the target of $\$ 50,000$ per QALY [41].

\subsubsection{Antihypertensive Therapy}

To attain guideline-recommended BP goals, antihypertensive pharmacological therapy is often prescribed for individuals with CAD. There are several antihypertensive drug classes such as beta-blockers, angiotensin-converting enzyme inhibitors (ACEIs), angiotensin II receptor blockers (ARBs), calcium channel blockers, diuretics, and others [35], each with their own mechanism of lowering BP. In addition, the efficacy and effectiveness of beta-blockers [42, 43] and ACEIs/ARBs [44-48] in reducing cardiovascular events among individuals with CAD have been proven.

\subsubsection{The Importance of Beta-blockers, ACEIs, and ARBs}

However, there is no convincing evidence on the superiority of the cardio-protective effect of one single antihypertensive drug class over the others. The choice of antihypertensive drug class is usually guided by the compelling indication [49, 50]. Beta-blockers are usually the first-line antihypertensive medication for individuals with $\mathrm{CAD}$ and are recommended for CAD patients [51]. Beta-blockers have also shown to be cost-effective in elderly patients [52]. As it has been established that ACEIs reduce the risk of death, MI, diabetes, and renal problems, ACEIs are recommended for individuals with CAD, specifically for those with diabetes to lower the risk of heart attack or heart failure. ARBs are prescribed to patients who cannot tolerate ACEIs [36]. ACEIs have demonstrated cost-effective in the elderly Australian population; the incremental cost for QALY was reported to be 29,535 Australian dollars, which is much lower than the target of \$50,000 per QALY [53].

Because of the broad benefits of these agents, the American College of Cardiology Foundation and the American Heart Association recommend that statins and antihypertensive medications be initiated and maintained for the secondary prevention of $C A D$ [27].

In summary, pharmacotherapy with statins and ACEIs/ARBs/beta-blockers is the mainstay of the effective CAD management.

\subsection{CAD Management and the Role of Incident Cancer}

It has been well documented that those with CAD have other co-existing conditions or at risk for developing new chronic conditions [54] and these conditions may compromise survival. Adults with CAD are more likely to develop other chronic conditions such as cancer, stroke, and congestive heart failure. Using the Duke Databank for Cardiovascular Diseases, Sachdev et al (2004) reported that co-existing chronic conditions were very common and 
were associated with poor survival among individuals with CAD who had undergone medical treatment for CAD and followed for an average of 13.6 years [55].

Of these, the subsequent presence of incident cancer among individuals with CAD is especially important for many reasons: (i) CAD and cancer have shared risk factors and unique pathophysiology; (ii) adults with CAD are more likely to develop cancer; (iii) there can be negative effects of some cancer treatments on cardiovascular health; (iv) as both CAD and cancer are life-threatening conditions, the co-management of these conditions is especially challenging; and (v) both conditions are very costly to treat and manage and such heavy financial burden has implications for payers, healthcare providers and patients.

\subsubsection{Shared Risk Factors}

There is evidence that cardiovascular diseases including CAD and cancer share many biological (example: inflammation, oxidative stress, hyperglycemia, hypertension, hypertriglyceridemia) and behavioral risk factors such as obesity, tobacco use, and poor diet. Although the pathways between CAD and cancer are multifaceted and complex, these shared risk factors may lead to the development of cancer in individuals with CAD and vice versa [56]. For example, inflammation has been shown to mediate all stages (from initiation to progression, and thrombosis) of atherosclerosis, a precursor to CAD. Similarly, the role of inflammation in promoting carcinogenesis and tumor progression is well established, where inflammation promotes malignant transformation of cells, carcinogenesis, and its progression [56].

\subsubsection{CAD as a Risk Factor for Cancer}

Some studies have provided evidence of CAD as a risk factor for development of cancer. The findings from the REDUCE study demonstrated that CAD was associated with a 35\% increased risk of developing prostate cancer [57]. An elevated risk of prostate cancer (adjusted odds ratio, 2.00; 95\% confidence interval, 1.18-3.39) has been reported among adults with CAD [58]. Diagnosis of CAD has also been associated with increased risk of colorectal cancer; Chan et al (2007) reported significantly $(P<0.001)$ higher prevalence of colorectal cancer in those with CAD (34.0\%) compared to their counterparts (18.8\%) [59].

\subsubsection{Negative Effects of Some Cancer Treatments on Cardiovascular Health}

While the goal of CAD management is to reduce coronary events, cancer treatments such as radiotherapy, chemotherapy, and hormone therapy can accelerate or worsen pre-existing cardiac disease [60-62]. Breast cancer treatments include chemotherapy (e.g., anthracyclines), surgery, radiation therapy, hormone therapy (e.g., tamoxifen), and targeted therapies (e.g., trastuzumab). Radiation therapy is of particular concern because of proximity to the heart, which can increase the risk of CAD or worsen pre-existing CAD resulting in major coronary events (e.g., heart attack) and even death. Anthracyclines and trastuzumab typically result in clinical heart failure or heart attack [63-65]. Androgen deprivation therapy (ADT) is the key therapeutic approach for metastatic prostate cancer and is also used in patients with a manifestation of disseminated disease with rising or elevated serum prostate-specific antigen. However, use of ADT increases the risk of CAD, the risk being further higher in those 
with pre-existing CAD [66]. Colorectal cancer treatment comprises of surgery, chemotherapy (e.g., capacetabine, 5fluorouracil, bevacizumab, etc.), and radiation therapy. The cardiotoxicity is mainly attributed to chemotherapeutic agents used for treating advanced and/or metastatic colorectal cancer. For example, patients receiving 5-fluorouracil have $45 \%$ risk of angina and 22\% risk of MI. Capecitabine is metabolized to 5-fluorouracil, thereby results in cardiotoxicity [67].

\subsubsection{Dominant Condition as the Driver of Treatments: CAD or Cancer?}

Management of CAD in cancer patients is very challenging. Despite advancements in treatments, both CAD and cancer continue to be the leading causes of death [68]. Therefore, in medical decision making, in which lifethreatening conditions are prioritized, cancer can be considered as dominant condition that "eclipses" the management of CAD [69], specifically among the elderly.

CAD-related care after cancer diagnosis may be prioritized because those with CAD may be at a higher risk for mortality compared to those without cancer. For example, it has been reported that women with breast cancer were at higher risk of mortality due to cardiovascular diseases as compared to women without breast cancer [70]. Furthermore, to reduce the negative effects of cancer treatment on cardiovascular health, providers may pay increased attention to CAD management, specifically the prescription of statins and ACEIs/ARBs/beta-blockers. A meta-analysis of randomized clinical trials and observational studies in general population indicated that statins, beta-blockers, and ACEIs/ARBs can reduce cancer treatment-related cardiotoxicity. Cardiac events were significantly reduced with beta-blockers ( $\mathrm{RR}=0.31$ [95\% CI 0.16-0.63], $P=0.001)$, statins $(\mathrm{RR}=0.31$ [95\% CI 0.13-0.77], $P=0.01$ ) and ACEIs/ARBs (RR = 0.11 [95\% CI 0.04-0.29], $P<0.0001$ ) [71]. In addition, it is recommended that patients with unstable angina or acute MI should be evaluated and stabilized before they undergo cancer-related surgery [72] suggesting that CAD management will be prioritized before cancer treatment.

On the other hand, cancer treatment may be prioritized because of "life-threatening" nature of the disease. Indeed, a patient with pre-existing CAD and incident cancer can be considered as a prototype example of a complex patient, as defined by Agency for Healthcare Research and Quality "two or more chronic conditions in which each condition might influence the care of the other condition, either through a limitation on life expectancy imposed by one of the conditions or because these two conditions generally entail therapies which are likely to have interactions with each other, or thirdly wherein one of these conditions might be requiring therapies that are contraindicated by the presence of the other conditions” [73].

\subsubsection{Healthcare Expenditures: CAD and Cancer}

Both CAD and cancer are very costly to treat and manage and such heavy financial burden has implications for payers, healthcare providers and patients. It has already been established that heart disease is a very expensive medical condition [74]. Individuals with cancer also have higher expenditures as compared to those without cancer [74]. In terms of average per-capita healthcare expenditures, heart disease and cancer were the first and second most expensive conditions in the US [75]. Among all heart diseases, CAD is the most expensive condition [26]. CADrelated expenditures are projected to increase from \$135.7 billion in 2010 to $\$ 161.4$ billion in 2020 [26]. Direct costs 
of cancer are also projected to increase from \$124 billion in 2010 to \$158 billion in 2020 [76]. Diagnosis of incident cancer in individuals with pre-existing CAD can further result in increased costs resulting from cancer treatmentrelated cardiotoxicity.

In summary, incident cancer may play a crucial role in the management and CAD-related outcomes and costs, therefore, studies are needed to elucidate the relationship between incident cancer and pharmacotherapy for CAD management, CAD-related outcomes and healthcare expenditures.

\subsection{Significance of the Study}

To date, few studies have examined the management of CAD in elderly patients with pre-existing CAD and incident cancer. Such studies are warranted because of: (i) the relatively high burden of CAD as compared to other heart diseases; (ii) substantial disease burden of CAD among the elderly; (iii) high prevalence of CAD and cancer among the elderly; (iv) benefits of CAD-related pharmacotherapy for both CAD and cancer survival; and (v) cost implications of CAD and cancer.

\subsubsection{Burden of CAD in Comparison to Other Heart Diseases}

Among all the cardiac conditions, CAD is the most common form of heart disease in the US adults ( $\geq 18$ years). In 2015, prevalence of CAD was 8.3\% compared to other cardiac conditions, i.e., heart failure (3.0\%) and stroke (3.4\%) [26]. Based on National Center for Health Statistics (2008), CAD accounted for $49.9 \%$ of all cardiovascular deaths compared to stroke (16.5\%), other cardiovascular disease (15.6\%), hypertensive diseases (7.5\%), heart failure (7.0\%), and diseases of arteries (3.4\%) [77]. Further, CAD accounted for $\$ 46.8$ billion in direct costs compared to heart failure ( $\$ 32.4$ billion) and stroke (\$38.0 billion) [26].

\subsubsection{Burden of CAD in the Elderly Population}

The prevalence of CAD is highest among individuals 65 years and older; $19.8 \%$ of this population having CAD versus younger $8.4 \%$ among those under age 65 years [6]. Among 60 to 79 years' age group, approximately $25 \%$ of men and $16 \%$ of women have CAD, and the prevalence further rises in those 80 years and older, i.e., 37\% and 23\% among men and women, respectively [27]. In addition, about $80 \%$ of CAD-related mortality is reported in among elderly population (65 years and older) [4].

The rates of mortality and morbidity further increase with increase in age. CAD accounts for one-third to onehalf of all deaths in the elderly Americans and about one in every four elderly CAD-related functional disabilities. In those above 65 years of age, about $8 \%$ die annually following MI and at least one half experience fatality related to other coronary events within 10 years. This mortality rate is double than that of individuals younger than 65 years of age [78]. In approximately $50 \%$ of patients, angina is the initial manifestation of CAD and the incidence of angina rises with increase in age [4]. Two population-based studies on elderly population (65 years and older) reported similar annual rates of MI in patients with symptoms of angina, i.e., 3\% and 3.5\% per year [79, 80]. 
Given the higher prevalence of CAD in the elderly population, nearly three-quarters of total expenditures for CAD can be attributed to the individuals aged 65 years and older [81]. Moreover, with the increase in the older adult population in the US, it has been projected that, relative to year 2010 (\$35.7 billion), expenditures for the treatment of CAD will increase 66\% by the year 2030 ( $\$ 106.4$ billion) [26], and the fastest growth rate in costs will be among the elderly population ( $\geq 65$ years) [26].

CAD is the second leading cause of disability among older adults, and this attributes to lower self-reported HRQoL in this population. Cardiovascular complication resulting from unstable CAD negatively affect physical functioning and their ability to perform routine activities of daily living [82]. Although not specific to CAD, an association between cardiovascular disease and frailty, resulting in increased risk of disability [83] has been reported. Results from the cardiovascular health study indicated that the presence of cardiovascular disease in elderly participants was associated with a loss of approximately 5.6 years and 6.5 years of "successful” life (i.e. with good health and function) in men and women, respectively [84].

\subsubsection{CAD and Cancer in the Elderly Population}

Age is the most powerful non-modifiable risk factor for both CAD and cancer; the incidence of both cancer and cardiovascular disease increases with age [85-87]. Prevalence estimates indicate that $77 \%$ of all cancers are diagnosed in those aged 55 years or older. Similarly, the risk of CAD increases after the age of 55 years among men and 65 years among women [88, 89]. Amongst older individuals diagnosed with cancer, heart disease is the most common pre-existing condition; about $20 \%$ of elderly individuals with newly diagnosed cancer have some form of pre-existing cardiovascular condition [90]. Further, both CAD and cancer are the leading cause of morbidity and mortality in elderly population.

Investigators from the CORONOR study reported that individuals with CAD have similar mortality rates as those of the general population [91] and among surgically treated individuals with CAD, the 10-year survival rate was estimated at 77\% [92]. Therefore, many individuals with CAD can survive to old age. As many types of cancers are often considered as the disease of the elderly, an individual may develop cancer after CAD.

\subsubsection{Statins, Beta-blockers, ACEIs, and ARBs and CAD-related Outcomes}

While beneficial effects of pharmacotherapy with statins and ACEIs/ARBs/beta-blockers for secondary prevention are proven for individuals with CAD, these benefits can be achieved and sustained only when individuals adhere to these medications over a period of time. Medication adherence, the "extent to which an individual takes medications as prescribed by their healthcare providers" [93], is associated with reduced risk of hospitalizations, recurrent coronary events, and cardiovascular mortality [94, 95]. Furthermore, adherence to both statins and antihypertensive therapies have been reported to reduce the relative risk of CAD mortality by $80 \%$ [96]. In addition, optimal adherence to these pharmacotherapies significantly reduces annual costs for secondary prevention of CAD; between $\$ 294$ and $\$ 868$ per patient, accounting to 10.1\% - 17.8\% cost reductions between adherent and nonadherent groups [94]. 


\subsubsection{Cost Implications of CAD and Cancer in the Elderly Population}

An overwhelming percent (96\%) of the expenditures among the elderly are due to multiple chronic conditions [97, 98]. Cancer and heart disease are two of the most expensive conditions [75]. As 84\% of the elderly over 65 years of age are covered by Medicare [99], this has implications for healthcare financing reforms. As policy makers and providers have priorities better health, better value and lower costs, it is important to understand the financial burden of CAD and cancer for program, policy planning, and allocation of resources.

To summarize, incident cancer plays a crucial role on CAD-related pharmacotherapy, outcomes, and expenditures.

\subsection{Need for the Study}

However, there is paucity of research on the impact of cancer on CAD-related pharmacotherapy, outcomes, and expenditures. There is a need for studies that exclusively focus on CAD-related pharmacotherapy, outcomes and expenditures among elderly fee-for-service (FFS) Medicare beneficiaries with incident breast, colorectal and prostate cancer because: (i) Medicare covers $84 \%$ of the elderly population and the primary payer of healthcare for the elderly; (ii) elderly share a disproportionate burden of CAD; and (iii) the cancer of breast, prostate, and colon/rectum, and lung/bronchus are the most common invasive cancers in the US.

\subsubsection{Medicare as the Primary Payer of CAD and/or Cancer}

Nearly $84 \%$ of the elderly are covered by Medicare. Payments made by Medicare for CAD are substantial. For example, in 2003, the Medicare program paid \$12.2 billion for CAD-related hospitalizations, including \$12,321 per discharge for acute MI and \$11,783 per discharge for coronary atherosclerosis [100]. Similarly, Medicare payments for treatment of cancer totaled \$29 billion in 2006. In addition, \$2 billion were spent on drugs to treat the sideeffects of chemotherapy [101].

\subsubsection{Disproportionate Burden of CAD among Elderly Population}

As discussed earlier, elderly population share a disproportionate burden of CAD [4]. Similarly, burden associated with cancer is also higher among elderly population [102]. Further, the prevalence of CAD is higher among elderly Medicare FFS beneficiaries, with 34\% of beneficiaries aged 65 years and older were diagnosed with CAD compared to $19 \%$ of younger beneficiaries (aged <65 years) [97, 103]. Similarly, prevalence of cancer is higher among elderly Medicare beneficiaries [101]. As Medicare is the primary payer for healthcare expenditures for elderly Medicare beneficiaries [104], with nearly 42.9 million enrolled (based on 2013 enrollment in Medicare Part A and/or B) [99], it is important to focus on this population.

\subsubsection{CAD and Common Cancers}

The cancer of breast, prostate, colon/rectum, and lung/bronchus are the most common invasive cancers in the US population. Based on the Centers for Disease Control and Prevention statistics (2011), breast, lung, and colorectal cancer have the highest incidence rates among all female cancer cases; rates per 100,000 women are 
122.0, 52.0, and 34.9, respectively. Prostate, lung, and colorectal cancer have the highest incidence rates among all male cancer cases; rates per 100,000 men are 128.3, 73.0, and 46.1, respectively [105]. Three cancer sites - breast, prostate, and colorectal cancers have been selected because of the availability of commonly used screening modalities and the existence of competing therapies. Further, with the currently available screening tests, these three cancers have a higher probability of diagnosis at an earlier stage compared to lung cancer, and thus improved survival rates. The estimated 5-year relative survival rate is $91 \%$ for women with breast cancer, $66 \%$ for patients with colorectal cancer, and $99 \%$ for patients diagnosed with prostate cancer, whereas it is only $18 \%$ for lung cancer [106]. These cancers are common among older adults ( $\geq 65$ years). Based on the Surveillance, Epidemiology, and End Results (SEER) Program 2008-2012 statistics, incidence of breast cancer (41.8\%), colorectal cancer (58.6\%), and prostate cancer is $41.8 \%, 48.6 \%$, and $56.7 \%$, respectively [107]. In addition, prevalence of MI and CAD in elderly population with breast, colorectal or prostate cancer is about $15.4 \%, 7.4 \%$ or $6.1 \%$ and $10.2 \%, 16.0 \%$ or $16.5 \%$, respectively [108-114]. Thus, CAD and incident cancer are highly prevalent in common cancers such as breast, colorectal and prostate cancers.

To summarize, studies on evaluating the relationship between incident cancer and CAD-related pharmacotherapy adherence, and expenditures and the broad benefits of CAD-related pharmacotherapy adherence on CAD-related outcomes among elderly cancer patients are needed for informing clinical practice, research, program planning, and allocation of resources. However, there are critical gaps in knowledge because of paucity of studies in this area. The present dissertation is aimed at addressing these aforementioned critical gaps in knowledge by focusing on a cohort of elderly Medicare beneficiaries with pre-existing CAD and a diagnosis of incident breast, colorectal or prostate cancer.

\subsection{Specific Aims}

The specific aims, objectives, and hypothesis of the dissertation are as follows.

Aim 1: Examine the relationship between incident cancer and adherence to statins and/or ACEIs/ARBs/betablockers among elderly fee-for-service Medicare beneficiaries with pre-existing CAD by comparing those with incident (breast, colorectal, and prostate) cancer and without cancer.

Hypothesis: Adherence to CAD-related concomitant pharmacotherapy will be lower among elderly Medicare FFS beneficiaries with CAD and incident cancer as compared to those with CAD and no cancer.

Aim 2: Examine relationship between adherence to both statins and ACEIs/ARBs/beta-blockers and CAD-related hospitalizations among elderly fee-for-service Medicare beneficiaries with pre-existing CAD and incident breast, colorectal, or prostate cancer, after controlling for cardiotoxic cancer treatments and other risk factors that may affect CAD-related hospitalizations.

Hypothesis: Better adherence to concomitant therapy will be associated with lower risk of CAD-related hospitalizations, after controlling for cancer treatment and other risk factors. 
Aim 3: Estimate the association between incident cancer and short-term CAD-related healthcare expenditures by comparing the elderly fee-for-service Medicare beneficiaries with pre-existing CAD and incident cancer to their counterparts with CAD and no cancer.

Hypothesis: The short-term CAD expenditures will be higher among those with CAD and incident cancer as compared to those with CAD and no cancer.

\subsection{Innovation and Unique Contribution of the Study}

Cardio-oncology or onco-cardiology is an emerging field and most of the existing evidence on management of heart diseases in individuals with cancer is based on clinical expert opinion. There is paucity of studies on the influence of cancer diagnosis on management of CAD. Our study will contribute to the nascent literature on management of CAD in patients with cancer. There are several facets of our study that makes it unique and important for understanding the impact of cancer on CAD related medication adherence, outcomes, and healthcare expenditures.

First, the current dissertation fills a critical knowledge gap on the management of chronic conditions among cancer survivors has been very limited. It is important to recognize ways in which pre-existing conditions could affect the health of cancer patients so that patient-centered care and individualized treatment can be achieved [115].

\section{Second, our study can illuminate the complex relationship between CAD management and incident cancer.} Prior studies have suggested that cardiovascular disease management is compromised in cancer patients. A study utilizing Medicare-linked database examined management of cardiovascular disease in older women ( $\geq 65$ years) with breast cancer and concluded that these women received inadequate treatment and care for heart problems. Their study findings indicate that about $12 \%$ of these older women with breast cancer developed heart failure within three years of cancer diagnosis and treatment. Further, the findings indicate that only $33 \%$ of these patients saw a cardiologist within 90 days of developing heart problems [116]. Another study, examined quality of care for chronic disease, such as cardiovascular disease, diabetes, and hypertension in cancer survivors, and concluded that the cancer survivors received inadequate care for these chronic conditions compared to those without cancer [117].

Third, this study is a population-based study utilizing "real-world" data. It has been pointed out by the investigators of the CLARIFY study among individuals with stable CAD that real-world studies on CAD are needed because "clinical trials or registries which often have stringent exclusion criteria, and do not adequately represent populations with stable CAD (in terms of age, comorbidity, and concomitant therapy)” [118]. The current dissertation highlights the importance of real-world studies.

Fourth, this dissertation focuses on elderly population and it is estimated that by 2050, there will be 83.7 million over the age of 65 [119]. Both CAD and cancer are highly prevalent in aging population. Further, it has been previously reported that elderly patients are under-represented in clinical trials, with individuals older than age 65 years representing only 38\% of enrolled patients, specifically those with comorbid conditions [120]. Understanding the influence of life events such as cancer diagnosis on management of pre-existing chronic conditions are important to the growing elderly population with multiple chronic conditions. Our proposed study is the next best alternative to clinical trials for understanding this relationship. 
Fifth, this dissertation focuses on elderly FFS Medicare beneficiaries. As discussed earlier, Medicare is the primary payer of healthcare for elderly in the US [104]. Therefore, Medicare bears the largest share of the clinical, humanistic, and economic burden in this population. The findings from this study will have implications for the various healthcare delivery and financing reforms such as the establishment of accountable care organizations, patient-centered medical home and others undertaken by the Centers for Medicare and Medicaid Services, which is responsible for setting policy priorities and healthcare payments.

Sixth, this dissertation can provide insight into co-management for multiple conditions. CAD and cancer share a unique relation. In general, it is difficult to manage multiple health conditions in elderly patients as treatment of one medical condition may interfere with treatment of other medical condition [73]. There is paucity of evidence on CAD management in patients with cancer. The study findings may be useful in understanding the influence of cancer on CAD management.

\subsection{Conceptual Framework}

The selection of variables was guided by conceptual frameworks and these differed by specific aims.

\subsubsection{Conceptual Framework: Aim 1}

A conceptual framework by Park and Jones was adapted for understanding influence of cancer on adherence to cardiovascular medications among elderly population. This model considers patient-related factors, health-system factors, provider factors, and external environment. This model has also been used previously by Gellad et al (2009) for understanding factors that affect adherence to medications in elderly population [121]. The conceptual framework is shown in Error! Reference source not found..

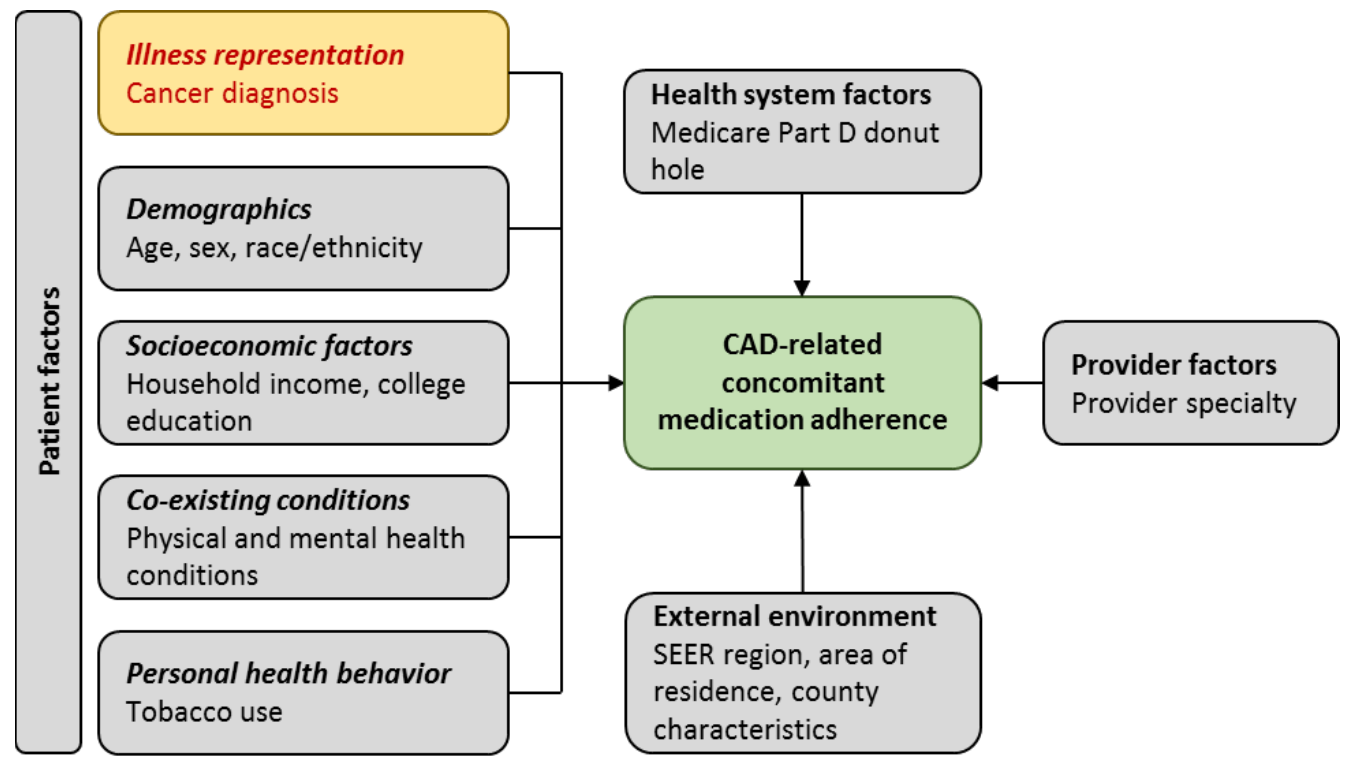

Figure 1.1 Conceptual framework adapted from Park and Jones for medication adherence.

Abbreviations - CAD: Coronary artery disease; SEER: Surveillance, Epidemiology, and End Results 


\subsubsection{Conceptual Framework: Aim 2 and Aim 3}

The Andersen behavioral model of health service use and outcomes was adapted for this study as it provides an appropriate framework for understanding the relationship between explanatory variables and outcomes. This framework also provides basis for selecting other independent variables that may influence CAD outcomes [122, 123].

This original model was developed by Andersen and his colleagues in 1968 and has been used to examine various types of healthcare utilization in elderly population with chronic conditions. It is a multi-level model that includes both individual and contextual determinants of healthcare utilization and categorizes these determinants into predisposing, enabling, and need factors, personal health practices/ health behavior, and external environment [124, 125].

The predisposing factors are the individual's demographic characteristics which decide their proclivity towards healthcare utilization. Factors such as age, sex, and race/ethnicity are "biological imperatives" that influence their unique predisposition towards use of healthcare services. The enabling factors include resources available to each individual that enable them to obtain services. In the context of this study, these factors include marital status, Medicaid coverage (insured, uninsured), education, income, and poverty status. The need-based factors at the individual level are those related to the perceived need for health services due to functional and health problems. In the context of this study, need-based variables include cancer type, stage of cancer, cancer treatment, comorbid chronic physical conditions, and mental health conditions. The personal health practices include tobacco use, alcohol abuse, and healthcare-seeking behavior. The external environment includes following variables for this study SEER region, area of residence, health professional shortage area (HPSA), county level number of oncologists and cardiologists, and centers for cardiovascular care. The conceptual framework for CAD-related hospitalizations as the outcome is shown in Figure 1.2 and for CAD-related healthcare expenditures as the outcome is shown in Figure $\mathbf{1 . 3}$ 


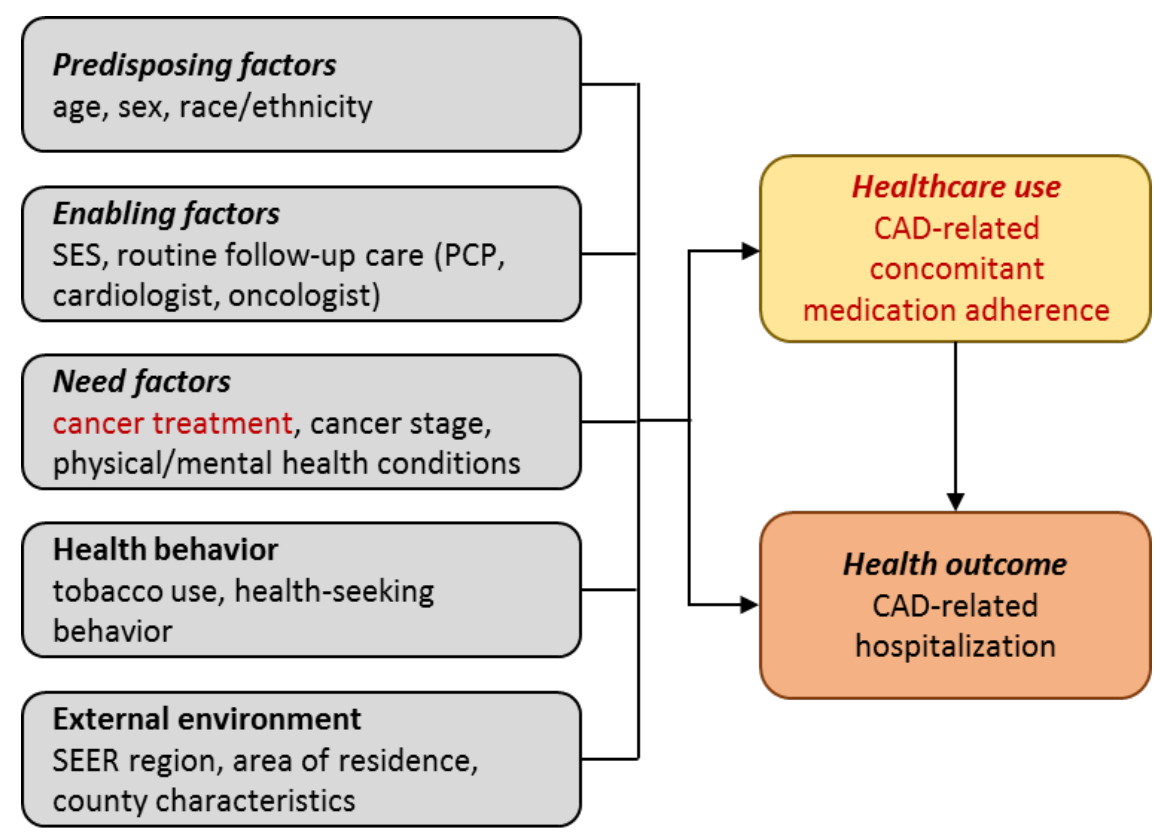

Figure 1.2 Conceptual framework adapted from the Andersen Behavioral Model of Health Service Use for medication adherence and health outcomes.

Abbreviations - CAD: Coronary artery disease; PCP: Primary care physician; SEER: Surveillance, Epidemiology, and End Results; SES: Socioeconomic status

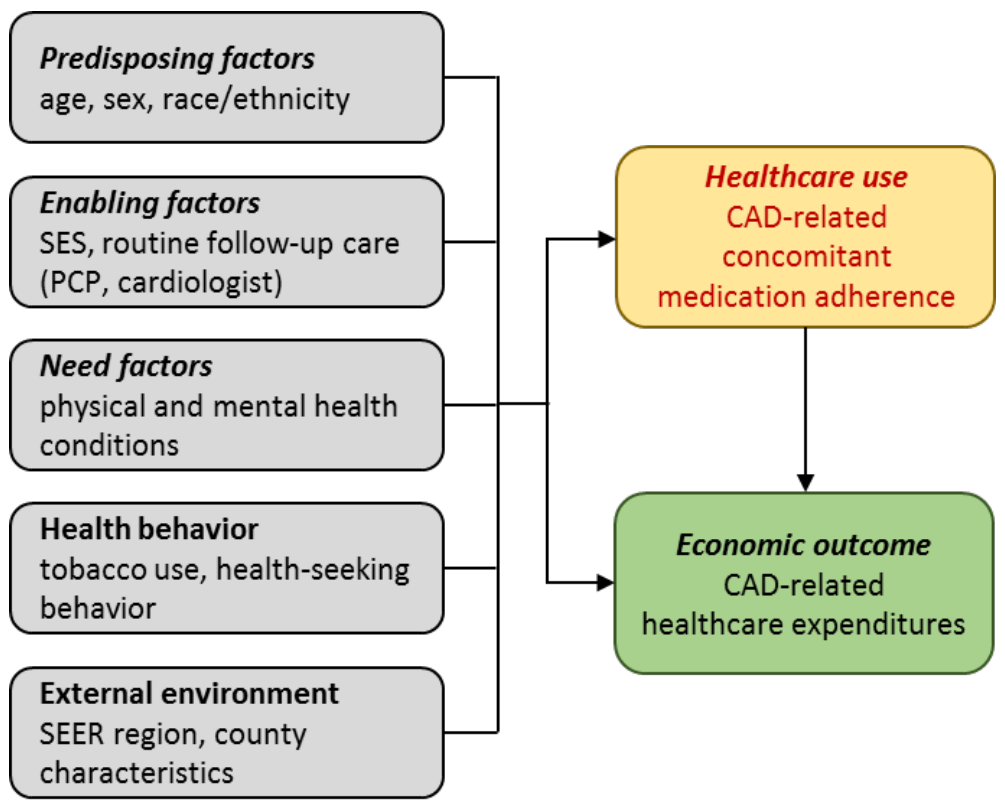

Figure 1.3 Conceptual framework adapted from the Andersen Behavioral Model of Health Service Use for medication adherence and economic outcome.

Abbreviations - CAD: Coronary artery disease; PCP: Primary care physician; SEER: Surveillance, Epidemiology, and End Results; SES: Socioeconomic status 


\subsection{Data Sources for the Study}

Data for the study population will be derived from the SEER-Medicare linked files. The comparison group of non-cancer patients will be derived from the non-cancer $5 \%$ sample files. The files will also be linked to the Area Health Resources File (AHRF), census tract files, and county health ranking files.

\subsubsection{SEER-Cancer Registry}

SEER program is an epidemiologic surveillance system consisting of population-based tumor registries that collects data on all incident cases of cancer that occur in persons residing in 18 SEER areas. These areas include Atlanta, Connecticut, Detroit, Hawaii, Iowa, New Mexico, San Francisco-Oakland, Seattle-Puget Sound, Utah, Los Angeles and San Jose-Monterey, Rural Georgia, Alaska Native, Greater California, Kentucky, Louisiana, New Jersey, and Greater Georgia. SEER data also consists of information about demographic characteristics of the patient, diagnosis date, cancer site, stage, and grade, and type of surgical treatment and radiation therapy provided within 4 months of diagnosis [126]. The Patient Entitlement and Diagnosis Summary File (PEDSF) contains all of this information from the SEER program.

\subsubsection{Medicare}

Medicare is the primary health insurance provider of US population above 65 years of age and thus, SEER data have been linked to Medicare claims. This database consists of the following files - Medicare Provider Analysis and Review (MEDPAR), the Carrier Claims (old name National Claims History (NCH) from physician/supplier), Outpatient, Home Health Agencies (HHA), Hospice, Durable Medical Equipment (DME), and Part D Event (PDE) files. The MEDPAR file includes hospital claims for all short- and long-term and skilled nursing facility stay. The $\mathrm{NCH}$ file contains claims from physicians and other non-institutional care providers and has procedure codes according to the Health Care Procedure Classification Code (HCPCS) and the Common Procedural Terminology, 4th Edition (CPT-4), and the International Classification of Diseases, Ninth Revision, Clinical Modification (ICD-9$\mathrm{CM}$ ) procedure codes with service dates. The outpatient file includes claims from institutional outpatient providers, including hospitals, rural health clinics, and mental health centers.

Part $A$ of the Medicare program consists of the following types of services - inpatient hospitalization, Skilled Nursing Facility (SNF), home health agency services (HHA) following hospital stay and hospice care. Part B of the Medicare program includes physician services, outpatient services, diagnostic test, DME, emergency room visits, home health care not following a hospital stay, laboratory services, and other medical services. Part D of the Medicare program covers prescription drugs. The Medicare started providing voluntary outpatient medications coverage (Part D) since January 2006, and hence Part D data is available only after 2006.

\subsubsection{SEER-Medicare}

The Medicare data files have been linked with PEDSF file of cancer cases from SEER using an algorithm based on the social security number, last name, first name and date of birth of an individual. Approximately $94 \%$ of the SEER cancer cases over age 65 years with Medicare enrollment have been linked using this method [126]. Based on 
the linkage, a common encrypted identification number is given to each enrollee in PEDSF and claims files. Each record represents an episode of hospital stay. These records can have up to 10 diagnoses (ICD-9-CM codes) for each date of admission and date of discharge.

\subsubsection{SEER Non-Cancer Medicare}

SEER non-cancer Medicare database also provides a 5\% non-cancer random sample from Medicare program in the same catchment areas as those in SEER program that can be used to compare cancer and non-cancer case. The Summarized Denominator file (SUMDENOM) provides demographic information. These beneficiaries are randomly selected based on the last two digits of their social security number [126]. The individuals who have been reported to SEER with an incident cancer are removed from this 5\% random sample. The demographic and other Medicare enrollment data for non-cancer cases are identical to those of cancer cases and can be linked with the cancer files using each beneficiary’s unique Medicare claim number [127].

\subsubsection{American Community Survey (ACS) Census Tract Files}

The ACS 2008 to 2012 census tract and census zip code files were linked to PEDSF files by geographic codes, i.e., state and county [128]. These files provided information on the census tract median household income and education level.

\subsubsection{AHRF File}

The AHRF is a publicly available data file provided by Department of Health and Human Services and contains county, state and national files. The AHRF provides more than 6,000 variables for each of the nation's counties. The AHRF contains information such as health facilities, health professions, and socioeconomic and environmental characteristics. The basic file contains geographic codes and descriptors that can be used to link it to other files and to aggregate counties into various geographic groupings [129]. This study will use the AHRF variables to measure health care infrastructure and socio-economic status of the counties that can influence medication adherence, CADrelated outcomes and healthcare expenditures.

\subsubsection{County Health Ranking File}

The county health ranking file was linked to PEDSF files by geographic codes, i.e., state and county. These files provide a snapshot of a community's health, such as rates of smoking, obesity, and other health status measures at the county-level [130]. These variables can be used as a proxy for external health environment that can influence medication adherence, CAD-related outcomes and healthcare expenditures. 


\section{CHAPTER 2}

\section{Incident Cancer and Adherence to Statins and Antihypertensive Medications for Coronary Artery Disease among Elderly Medicare Beneficiaries}

\subsection{Abstract}

Objectives: To examine the relationship between incident cancer and adherence to statins and angiotensinconverting enzyme inhibitors (ACEIs), angiotensin II receptor blockers (ARBs), or beta-blockers among elderly individuals with coronary artery disease (CAD).

Methods: This was a retrospective observational longitudinal study assessing elderly Medicare fee-for-service beneficiaries with pre-existing CAD and those with incident breast (BC), colorectal (CC), or prostate (PC) cancer (N $=12,096)$ and those with no cancer $(\mathrm{NC})(\mathrm{N}=42,805)$. Adherence to pharmacotherapy was measured every 120 days during the one-year pre- and one-year post-index period. Due to the overlap between cancer type and sex, cancer diagnosis was grouped into: (1) women with BC; (2) women with CC; (3) women with NC; (4) men with PC; (5) men with CC; and (6) men with NC. Medication adherence was categorized into five mutually exclusive groups: (1) adherent to both statins and ACEIs/ARBs/beta-blockers; (2) not adherent to both statins and ACEIs/ARBs/betablockers (reference group); (3) adherent to either statins or ACEIs/ARBs/beta-blockers; (4) use of one medication class and adherent to that class; and (5) use of one medication class and not adherent to that medication class. The adjusted relationship between incident cancer and medication adherence was analyzed using the generalized estimating equation. Inverse probability treatment weights were used to account for observed group differences between cancer and non-cancer groups.

Results: Only $28.9 \%$ of the elderly with CAD were adherent to both statins and ACEIs/ARBs/beta-blockers. In the adjusted analyses, women $[\mathrm{AOR}=0.70 ; 95 \% \mathrm{CI}=0.58,0.81 ; P<0.0001]$ and men $[\mathrm{AOR}=0.63 ; 95 \% \mathrm{CI}=0.51$, $0.75 ; P<0.0001]$ with CC and men with PC $[\mathrm{AOR}=0.92 ; 95 \% \mathrm{CI}=0.85,0.99 ; P=0.022]$ were significantly less likely to be adherent to both medication classes compared to women and men with NC, respectively. No significant differences in adherence to medications were observed among women with BC compared to women with NC. Even among those using single medication class, women $[\mathrm{AOR}=0.64 ; 95 \% \mathrm{CI}=0.50,0.79 ; P<0.0001]$ and men with CC $[\mathrm{AOR}=0.59 ; 95 \% \mathrm{CI}=0.42,0.76 ; P<0.0001]$ were significantly less likely to be adherent to that medication class compared to women and men with NC.

Conclusions: Adherence to evidence-based medications for CAD varied by cancer types. Elderly Medicare beneficiaries with CC or PC were less likely to be adherent to evidence-based medications for CAD. Future research needs to explore the effect of non-adherence to concomitant medications on health outcomes such as survival among patients with incident CC and PC. 


\subsection{Introduction}

The two main goals of coronary artery disease (CAD) management are reduction of symptoms and ischemia as well as prevention of myocardial infarction and death [29]. The use of angiotensin-converting enzyme inhibitors (ACEIs), angiotensin II receptor blockers (ARBs), beta-blockers, and statins has become the standard of treatment among individuals with CAD. These medication classes have proven to reduce the risk of major coronary events [27], hospitalizations for CAD and recurrent coronary events [94, 95], and cardiovascular mortality by 11-35\% [131, 132] through improving lipid and blood pressure control [94, 133]. Therefore, CAD management guidelines recommend initiating and maintaining these regimens indefinitely in adults with CAD [27, 134].

Further, adherence to combined use of statins and ACEIs/ARBs/beta-blockers have been reported to reduce the relative risk of CAD mortality by $80 \%$ [96]. On the contrary, non-adherence to statins has been shown to increase the risk of adverse cardiovascular events (myocardial infarction, heart failure, or stroke) and mortality [94]. Nevertheless, non-adherence to statins, beta-blockers, and ACEIs/ARBs is common in the elderly with cardiovascular disease [135-138]. A study on elderly Medicare beneficiaries discharged from the hospital after their first myocardial infarction reported that only 46.4\% were adherent to beta-blockers, ACEIs/ARBs, and statins [138].

While many patient, provider, and health-system factors affect medication adherence in the elderly [139-142], development of an additional chronic condition may also impact adherence. Since both CAD and cancer are lifethreatening conditions, it may be challenging to effectively manage both simultaneously. Some types of cancer can be considered as a dominant condition that “eclipse” the management of CAD [69], whereas other types of cancer with a higher expected cure rate, CAD-related care after cancer diagnosis may be particularly important in order to reduce the probability of CAD morbidity and mortality due to cancer-related treatments like surgery [72], chemotherapy, and radiation [60, 61]. Some medications designed for CAD may even have anti-cancer properties, as recent epidemiological studies suggest additional benefits of statins in terms of reduced cancer recurrence and cancer-specific mortality among individuals with breast [143], colorectal [144], and prostate cancer [145, 146].

However, there is paucity of studies examining adherence to CAD medications in the context of a new cancer diagnosis. Calip et al reported a decline in adherence to statins (average medication possession ration $(\mathrm{MPR})=0.78$ vs. 0.68; $P<0.001$ ) in the period just after breast cancer diagnosis [147], but the findings from this study may be limited because it did not include a non-cancer comparison group. Without a non-cancer comparison group, it is not known whether adherence decline is due to cancer or due to the passage of time. Furthermore, the findings may not be generalizable to other cardiovascular medications like ACEIs/ARBs/beta-blockers, or elderly patients. Data assessing medication adherence among the elderly is particularly limited [148], despite the fact that both cancer [102] and CAD [4] disproportionately affect the elderly. In the United States (US), the prevalence of CAD is higher among the elderly (19.8\%) compared to those under age 65 years (8.4\%) [6]. Nearly 80\% of CAD-related mortality is reported among the elderly population [4]. Similarly, breast, colorectal, and prostate cancer are very common among the elderly, with 42.3\%, 57.7\%, and 56.9\% suffering from these cancers, respectively [107]. Examination of adherence to statins and ACEIs/ARBs/beta-blockers among the elderly is also important for policy purposes because 
Medicare provides coverage for almost all of the elderly in the US [99] and Medicare payments for CAD [100] and cancer care [101] are substantial.

Therefore, the primary objective of the current study is to examine the relationship between incident cancer and adherence to statins and/or ACEIs/ARBs/beta-blockers among elderly Medicare beneficiaries with pre-existing CAD by comparing those with incident (breast, colorectal, and prostate) cancer and without cancer.

\subsection{Methods}

\subsubsection{Conceptual Framework}

The conceptual framework by Park and Jones [121, 149] was adapted to select factors that can influence adherence to pharmacotherapy among elderly patients with CAD (Figure 1.1). This framework posits that patientrelated factors (e.g., age, sex, race, census tract education level, poverty status, co-existing chronic physical and mental health conditions, tobacco and alcohol use), health-system (e.g., Medicare coverage gap), provider-related factors (e.g., visits to primary care and cardiologist), and environmental factors (e.g., Surveillance, Epidemiology and End Results [SEER] region, county metro status, community health clinics, county-level health professional shortage area [HPSA], and percent of cardiologists and oncologists in the county) affect medication adherence.

\subsubsection{Study Design}

The study utilized a retrospective observational longitudinal cohort design with 12-month pre- and 12-month post-index period (Figure 2.1). Pre- and post-index periods were defined based on cancer diagnosis dates for the cancer cohort. For the non-cancer cohort, pre- and post-index periods were derived using pseudo-diagnosis dates randomly selected from the dates of service. A 36-month window, divided into a 24-month baseline (for identification of CAD and baseline characteristics) and a 12-month (pre-index) periods were created. The post-index period consisted of 12 months after the index date. Adherence to statins and ACEIs/ARBs/beta-blockers and selected variables were measured repeatedly every 120 days during the pre- and post-index period, yielding a total of six repeated measures for each individual. Thus, $\mathrm{t}_{1}, \mathrm{t}_{2}$, and $\mathrm{t}_{3}$ represented the pre-index period and $\mathrm{t}_{4}, \mathrm{t}_{5}$, and $\mathrm{t}_{6}$ represented the post-index period.

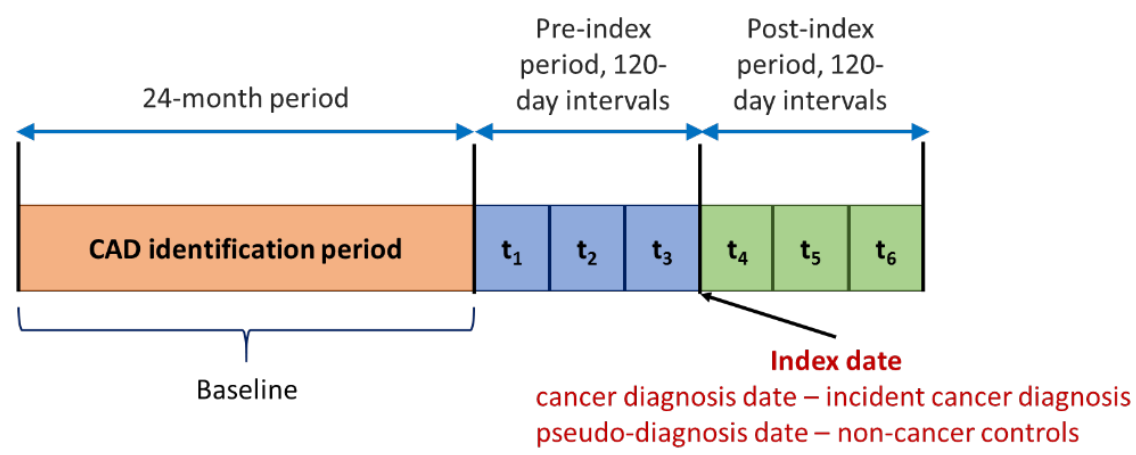

Figure 2.1. Schematic presentation of study design 
The longitudinal design with repeated measurement of adherence and other variables enabled tracking of the variability of adherence within and between individuals. By including a non-cancer comparison group, the study avoids the maturation threat to internal validity (i.e. the processes within subjects which act as a function of the passage of time).

\subsubsection{Data Sources}

Linked data from the following sources was utilized: 1) the SEER registries; 2) Medicare claims linked with the SEER registries; 3) 5\% non-cancer random sample of Medicare beneficiaries in SEER region; 4) the American Community Survey (ACS), and 5) the Area Health Resource Files (AHRF). SEER program is an epidemiologic surveillance system consisting of population-based tumor registries that collects data on all incident cases of cancer that occur in persons residing in 18 SEER areas. SEER data also consists of information about demographic characteristics of the patient, diagnosis date, cancer site, stage, and grade, and type of surgical treatment and radiation therapy provided within four months of diagnosis [126]. Individuals without cancer were identified from the $5 \%$ non-cancer random sample of Medicare beneficiaries living in same catchment areas as those in SEER program. These beneficiaries were randomly selected based on the last two digits of their social security number [126]. We derived information on the census-tract level poverty status and education from the ACS [128]. The individuals who have been reported to SEER with an incident cancer are removed from this $5 \%$ random sample. County-level healthcare environment factors (community health clinics, HPSA, and percent of cardiologists and oncologists in the county) were derived from the AHRF [129].

\subsubsection{Study Population}

The study population comprised individuals with pre-existing CAD and those with incident breast, colorectal, or prostate cancer and those without cancer. Individuals with CAD were identified using a validated algorithm developed by the Centers for Medicare and Medicaid Services (CMS) [150] to identify individuals with pre-existing CAD. The algorithm used the International Classifications of Diseases $-9^{\text {th }}$ edition, Clinical Modification (ICD-9$\mathrm{CM}$ ) codes. Individuals with at least one inpatient, outpatient and carrier claims (clinician encounters only), or home health agency service Medicare claims with a primary or secondary diagnosis of CAD during the baseline were classified as having pre-existing CAD.

Cancer and No Cancer Comparison Groups: Individuals with incident cancer, January 2008 through December 2011, were identified using the International Classification of Diseases for Oncology, $3^{\text {rd }}$ Edition (ICD-O-3) codes from the SEER registries. Individuals without cancer were identified from the 5\% non-cancer Medicare claims from the SEER regions.

Other inclusion criteria were age 68 years or older, alive with continuous FFS Medicare Part A, B enrollment during the entire study period, continuous part D enrollment during the pre- and post-index period, no missing information on county, and who had two or more prescriptions for either statins or two prescriptions for any or combinations of ACEIs, ARBs, or beta-blockers. In the non-cancer cohort, individuals with end-stage renal disease were excluded prior to diagnosis date assignments. In the cancer cohort, individuals with missing data for type and 
stage of cancer and those who were diagnosed with cancer through autopsy or death certificate were excluded. The selection criteria are presented in Figure 2.2.

\subsubsection{Measures}

2.3.5.1 Dependent Variable: Adherence to Statins, ACEIs/ARB/Beta-blockers

As this paper focuses on adherence over a long period of time, proportion of days covered (PDC) measure was used. PDC has been recommended by the Pharmacy Quality Alliance [151] and used by the CMS in its rating of insurance plans [152]. Statins, ACEIs, ARBs, and beta-blockers were identified using the generic names in the Part D Prescription Drug Event files. PDC of these medication classes were calculated only among those who filled at least two prescriptions for either statins or two prescriptions for ACEIs, ARBs, or beta-blockers. PDC was calculated as number of days of medication supplied during each time period (example: $\mathrm{t}_{1}$ ) divided by the number of days in each time period (i.e. 120 days). For those with a drug regimen (i.e. statins and ACEIs/ARBs/beta-blockers), PDC considers days within a particular period when an individual is covered for both statins and ACEIs/ARBs/betablockers. PDC ranges from 0 to 1 . Individuals with PDC $\geq 80 \%$ were considered as adherent and those with PDC $<80 \%$ as non-adherent [153].

We combined the adherence indicator (yes/no) and the type of drug regimens and classified individuals with CAD into five mutually exclusive groups: (1) adherent to both statins and ACEIs/ARBs/beta-blockers; (2) not adherent to both statins and ACEIs/ARBs/beta blockers; (3) adherent to either statins or ACEIs/ARBs/beta-blockers; (4) use of one medication class and adherent to that class; and (5) use of one medication class and not adherent to that medication class. This provides more granularity to the understanding of medication adherence based on use and adherence.

\subsubsection{Key Independent Variable: Cancer Type and Sex}

As the study included sex-specific cancers (breast and prostate cancer), the key independent variable was categorized into six mutually exclusive groups: women with breast cancer (BCW), women with colorectal cancer (CCW), women with no cancer (NCW), men with prostate cancer (PCM), men with colorectal cancer (CCM), and men with no cancer (NCM). 


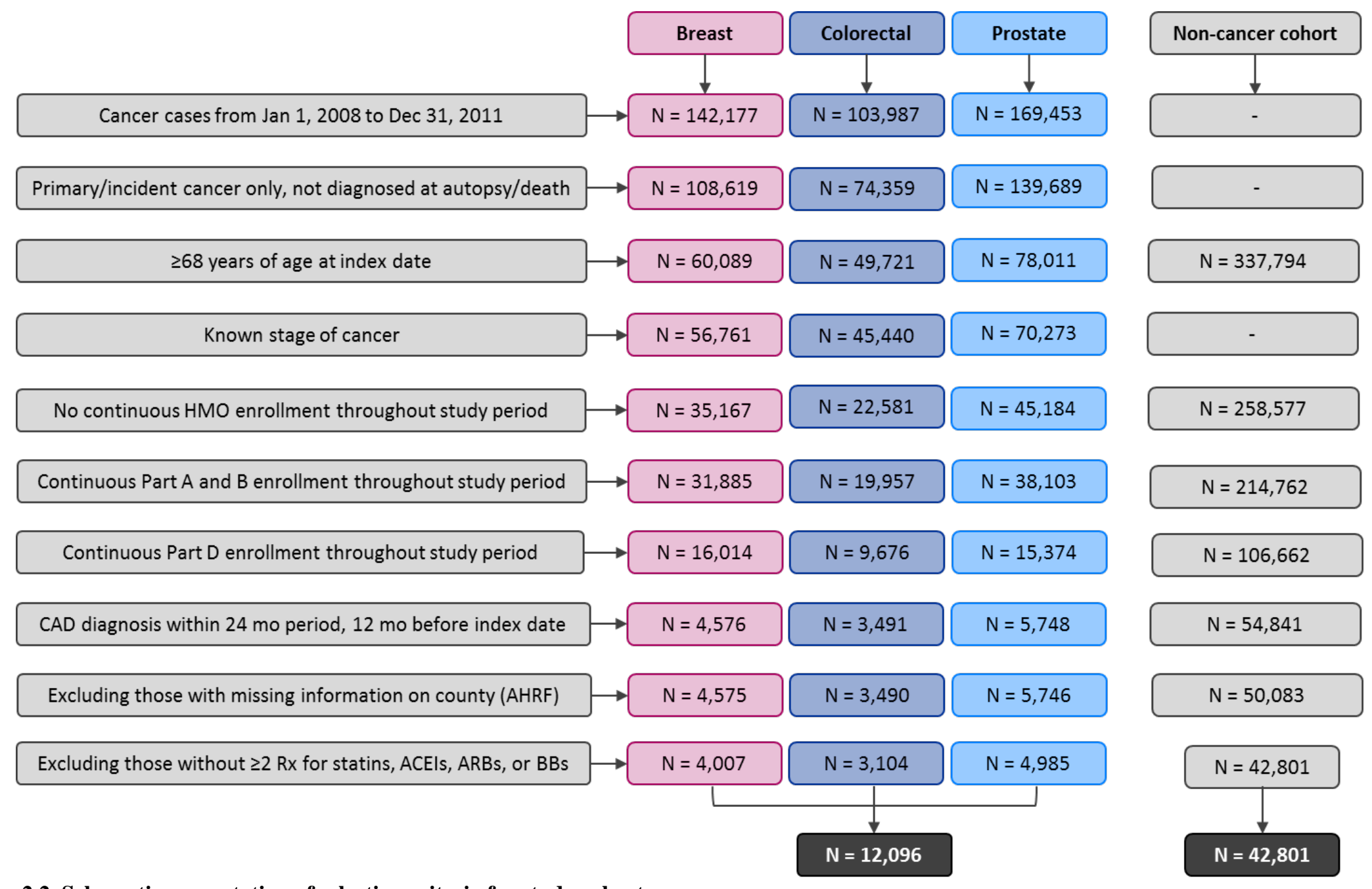

Figure 2.2. Schematic presentation of selection criteria for study cohort.

Abbreviations - ACEIs: Angiotensin-converting enzyme inhibitors; ARBs: Angiotensin II receptor blockers; BB: Beta-blockers; CAD: Coronary artery disease; HMO: Health Maintenance Organization; $\mathrm{R}_{\mathrm{x}}$ : Prescription

In the non-cancer cohort, individuals with end-stage renal disease were excluded prior to diagnosis date assignments. 


\subsubsection{Other Independent Variables}

\section{Patient-related factors}

These were time-invariant except when specified otherwise and were measured during baseline. These included (i) demographics - age in years measured at index month of incident cancer diagnosis, sex, and race/ethnicity; (ii) census-tract socioeconomic status - poverty status and education level; and (iii) CAD severity - a proxy measure was used for CAD severity based on the CMS hierarchical condition category (HCC) classification system. Based on the CMS risk adjustment model, each of the HCC codes for CAD was assigned specific score based on the risk and severity [154], ranging from 0.231 to 0.349 . Thus, higher scores represented severe manifestations of CAD.

Other physical and mental health conditions were selected based on the suggestions of the Multiple Chronic Conditions working group [155]. These were categorized into concordant (cardiac arrhythmias, congestive heart failure, diabetes, hyperlipidemia, hypertension, and stroke) and discordant (dementia, asthma, chronic obstructive pulmonary disease, arthritis, hepatitis, human immunodeficiency virus, and osteoporosis) conditions. Mental health conditions included severe mental illness (measured at baseline) and anxiety, depression, tobacco use, and alcohol use (measured every 120 days).

\section{Health-system factors}

Medicare Part D coverage gap was measured every 120 days. Part D plans typically start with an annual deductible, after which Medicare pays for the prescription drugs up to an initial coverage limit with certain coinsurance associated with those payments. The plan stops coverage once an individual reaches the annual coverage limit until the cost of prescription drugs reaches to catastrophic limit. The phase between the annual coverage limit and catastrophic coverage is called "donut hole" or "Medication coverage gap". During this period, enrollees have to bear the entire prescription drug costs. [156]. Based on their status of coverage, patients were categorized into three groups - not reached, reached, exceeded donut hole.

\section{Provider-related factors}

This included visit to primary care physician or cardiologist (yes/no), measured every 120 days.

\section{Environmental factors}

This domain included SEER region - Northeast (Connecticut, New Jersey), South (Kentucky, Louisiana, Atlanta, rural Georgia, and Greater Georgia), North-central (Detroit, Iowa), and West (San Francisco, Hawaii, New Mexico, Seattle, Utah, San Jose, Los Angeles, Greater California), county metro status (metro and non-metro), based on urban/rural continuum codes from the AHRF, community health resources - (county-level community health clinics, county-level health professional shortage area (HPSA), and percent cardiologists and oncologists in the county). These were measured at baseline.

\subsubsection{Statistical Analyses}


Unadjusted subgroup differences in time-invariant characteristics between cancer and non-cancer cohorts were tested with chi-square statistics. As medication adherence was measured every 120-days during pre- and post-index period, each individual had six observations. These six observations were not independent and applying standard regression techniques can lead to misleading results. Therefore, the unadjusted and adjusted relationship between cancer and medication adherence were analyzed with a repeated measures design using the generalized linear mixed models (GLMM), which can accommodate both linear and non-linear outcome variables. Mixed-effects models are very useful in modeling both within and between subject variations. Some non-linear models that can be considered for repeated measures include population-averaged (PA) and subject-specific (SS) models [157]. For categorical outcomes, the generalized estimating equations (GEE) is often used to model PA effects and random-effects logistic regressions can be used to estimate SS effects. Under the GEE approach, the correlation between repeated measures within an individual is accounted by a robust estimation, even when the dependency is miss-specified [158]. In this study, the PA approach was chosen because the objective is to estimate the average differences in adherence between cancer and non-cancer cohorts.

The adjusted GEE model controlled for time, age, race/ethnicity, census tract education level, poverty status, chronic physical and mental health conditions, tobacco use, alcohol use, Medicare coverage gap, visits to primary care and/or cardiologist, SEER region, county metro status, community health clinics, county-level HPSA, and percent of cardiologists and oncologists in the county.

\subsubsection{Inverse Probability Treatment Weights (IPTW)}

Our preliminary analyses indicated that there were significant group differences in sex, age, race/ethnicity, SEER region, and index year between the cancer and non-cancer cohorts. Therefore, we derived the IPTW and used these weights to adjust for these observed differences between cancer and non-cancer cohorts. We derived IPTW from the probability of cancer or no cancer based on a logistic regression on cancer and non-cancer with sex, age, race/ethnicity, SEER region, and index year as independent variables. The inverse probabilities of cancer or no cancer were used as weights in medication adherence analyses. Such weighting allows balancing of sex, age, race/ethnicity, SEER region, and index year between the cancer and non-cancer cohorts. Table 2.1 summarizes the findings from the logistic regression model used to calculate IPTW.

\subsection{Results}

\subsubsection{Characteristics of the Study Population Before and After IPTW Adjustment}

The study population comprised 54,901 elderly FFS Medicare beneficiaries with pre-existing CAD (12,096 with cancer and 42,805 without cancer). Table 2.2 summarizes the characteristics of cancer and non-cancer cohorts before and after IPTW adjustment. Prior to adjustment, those with cancer were less likely to be women than men (47.2\%\% vs 62.5\%), more likely to be younger than older (example: 80 or older) (66.3\% vs $57.2 \%$ ), and more likely to be Whites than African Americans, Hispanics, or other groups (82.1\% vs 77.8\%). After adjusting with IPTW, there were no significant differences in sex, age, race/ethnicity, SEER region, and index year between the cancer and non-cancer cohorts. 
Table 2.1. Logistic Regression Models used to derive IPTW-Cancer and $5 \%$ Non-Cancer Sample

\begin{tabular}{|c|c|c|c|}
\hline Characteristics & OR & $95 \% \mathrm{CI}$ & Significance \\
\hline \multicolumn{4}{|l|}{ Age groups } \\
\hline $68-70$ years & 1.03 & {$[0.97,1.10]$} & \\
\hline 71-74 years & 1.33 & {$[1.26,1.41]$} & $* * *$ \\
\hline 75-79 years & 1.36 & {$[1.29,1.44]$} & *** \\
\hline 80 years or older & \multicolumn{3}{|c|}{ Reference } \\
\hline \multicolumn{4}{|l|}{ Sex } \\
\hline Female & 0.52 & {$[0.50,0.55]$} & *** \\
\hline Male & \multicolumn{3}{|c|}{ Reference } \\
\hline \multicolumn{4}{|l|}{ Race/Ethnicity } \\
\hline African Americans & 0.96 & {$[0.89,1.04]$} & \\
\hline Hispanics & 0.61 & {$[0.54,0.69]$} & $* * *$ \\
\hline Others & 0.57 & {$[0.52,0.62]$} & $* * *$ \\
\hline Whites & \multicolumn{3}{|c|}{ Reference } \\
\hline \multicolumn{4}{|l|}{ SEER Region } \\
\hline Northeast & 1.13 & {$[1.06,1.19]$} & $* * *$ \\
\hline South & 0.98 & {$[0.93,1.04]$} & \\
\hline North-central & 1.25 & {$[1.17,1.34]$} & *** \\
\hline West & \multicolumn{3}{|c|}{ Reference } \\
\hline \multicolumn{4}{|l|}{ Index year } \\
\hline 2008 & 1.07 & {$[1.00,1.13]$} & * \\
\hline 2009 & 1.04 & {$[0.98,1.10]$} & \\
\hline 2010 & 0.98 & {$[0.93,1.04]$} & \\
\hline 2011 & & Refer & \\
\hline
\end{tabular}

Asterisks represent significant differences in study population characteristics (time-invariant), non-cancer group (reference category) for dependent variable.

${ }^{* * *} \mathrm{p}<0.001 ; * * 0.001 \leq \mathrm{p}<0.01 ; * 0.01 \leq \mathrm{p}<0.05$.

\section{$\underline{\text { Abbreviations }}$}

IPTW: Inverse probability of treatment weighting; OR: Odds ratio 
Table 2.2. Number, Percent, and Weighted Percent of Patient-level Characteristics by Presence of Cancer

\begin{tabular}{|c|c|c|c|c|c|c|c|c|}
\hline Characteristics & \multicolumn{5}{|c|}{ Before IPTW } & \multicolumn{3}{|c|}{ After IPTW } \\
\hline Age groups & & & & & $* * *$ & & & NS \\
\hline $71-74$ years & 2,887 & 23.9 & 8,186 & 19.1 & & 19.8 & 20.1 & \\
\hline 75-79 years & 3,158 & 26.1 & 9,423 & 22.0 & & 22.8 & 22.9 & \\
\hline 80 years and older & 4,068 & 33.6 & 18,336 & 42.8 & & 41.5 & 40.9 & \\
\hline Male & 6,384 & 52.8 & 16,041 & 37.5 & & 40.6 & 40.8 & \\
\hline Race/Ethnicity & & & & & & & & NS \\
\hline Whites & 9,928 & 82.1 & 33,308 & 77.8 & $* * *$ & 78.8 & 78.8 & \\
\hline African Americans & 1,013 & 8.4 & 3,538 & 8.3 & & 8.2 & 8.3 & \\
\hline Hispanics & 319 & 2.6 & 1,687 & 3.9 & & 3.7 & 3.7 & \\
\hline North-central & 1,691 & 14 & 5,029 & 11.7 & & 12.2 & 12.2 & \\
\hline West & 4,679 & 38.7 & 17,792 & 41.6 & & 41 & 40.9 & \\
\hline Index year & & & & & * & & & NS \\
\hline 2008 & 2,966 & 24.5 & 10,001 & 23.4 & & 23.2 & 23.6 & \\
\hline 2009 & 2,975 & 24.6 & 10,480 & 24.5 & & 24.5 & 24.5 & \\
\hline 2010 & 2,992 & 24.7 & 11,133 & 26.0 & & 26 & 25.8 & \\
\hline 2011 & 3,163 & 26.1 & 11,191 & 26.1 & & 26.3 & 26.1 & \\
\hline
\end{tabular}

Asterisks represent significant differences in time-invariant patient-level characteristics based on chi-square tests

$* * * \mathrm{p}<0.001 ; * * 0.001 \leq \mathrm{p}<0.01 ; * 0.01 \leq \mathrm{p}<0.05$. Column percentages are reported.

Abbreviations

ACEI: Angiotensin-converting enzyme inhibitors; ARB: Angiotensin II receptor blockers; CAD: Coronary artery disease; IPTW: inverse probability treatment weighting; Sig: Significance 


\subsubsection{Unadjusted and Adjusted Relationship between Cancer and Adherence to Statins and/or ACEIs/ARBs/Beta-blockers}

Table 2.3 summarizes the number and weighted percentage of medication adherence categories among cancer and non-cancer cohorts. Overall, only 29.1\% were adherent to both statins and ACEIs, ARBs, or beta-blockers and $14.6 \%$ were not adherent to both of these medication classes. Even among those with single medication class, $14.7 \%$ were not adherent. Table 2.3 also displays the odds ratios and 95\% confidence intervals (CI) from the unadjusted GEE models on adherence. The significance of the association was derived from the unadjusted GEE models. The reference group for the dependent variable was "adherence to both statins and ACEIs/ARBs/beta-blockers”. For ease of interpretation, we summarize the findings by cancer and no cancer differences, sex differences, and cancer type differences in adherence.

\subsubsection{Cancer versus No Cancer and Medication Adherence}

CCW were less likely to be adherent to both statins and ACEIs/ARBs/beta-blockers compared to NCW [OR = 0.72; 95\% CI $=0.61 ; 0.83, P<0.001]$. However, BCW were more likely to be adherent to both medication classes compared to NCW [OR = 1.09; 95\% CI = 1.01, 1.17; P<0.05]. Similarly, CCM were less likely to be adherent to both medication classes compared to NCM [OR $=0.66$; 95\% CI = 0.55, 0.78; $P<0.001]$. PCM were less likely to be adherent to both medication classes compared to NCM [OR $=0.92 ; 95 \% \mathrm{CI}=0.85 ; 0.99, P=0.026]$. Among those with single medication use, similar patterns were observed.

\subsubsection{Sex and Medication Adherence}

We compared NCW to NCM and CCW to CCM to infer sex differences in adherence categories. There were no significant differences in adherence to both medication classes by sex in the unadjusted. However, NCW [AOR = 1.10; $95 \% \mathrm{CI}=1.06,1.14]$ and CCW $[\mathrm{AOR}=1.21 ; 95 \% \mathrm{CI}=1.08,1.34]$ were more likely to be either adherent to one (out of two) medication class or adherent to one medication class among those with one medication class compared to NCM and CCM respectively. 
Table 2.3. Weighted Percent and Unadjusted GEE Models with IPTW on Medication Adherence Categories

\begin{tabular}{|c|c|c|c|c|c|}
\hline Characteristics & $\begin{array}{c}\text { ADH } 2 \text { Rx } \\
\text { (Weighted \%) } \\
\text { N = 80,957 } \\
\end{array}$ & $\begin{array}{c}\text { No ADH } 2 \text { Rx } \\
\text { (Weighted \%) } \\
\text { N = 41,016 } \\
\end{array}$ & $\begin{array}{c}\text { ADH } 1 \text { of } 2 \text { Rx } \\
\text { (Weighted \%) } \\
\quad N=60,167 \\
\end{array}$ & $\begin{array}{c}\text { ADH } 1 \mathrm{Rx} \\
\text { (Weighted \%) } \\
\mathrm{N}=55,536\end{array}$ & $\begin{array}{c}\text { No ADH } 1 \mathrm{Rx} \\
\text { (Weighted \%) } \\
\mathrm{N}=40,500 \\
\end{array}$ \\
\hline All & $29.1 \%$ & $14.7 \%$ & $21.6 \%$ & $20.0 \%$ & $14.6 \%$ \\
\hline \multicolumn{6}{|c|}{ Cancer and No Cancer Categories } \\
\hline $\mathrm{BCW}$ & 28.7 & 13.3 & 22.2 & 21.2 & 14.6 \\
\hline $\mathrm{CCW}$ & 24.5 & 16.9 & 23.5 & 18.7 & 16.4 \\
\hline NCW & 27.3 & 13.9 & 21 & 22.7 & 15.2 \\
\hline CCM & 27.3 & 19.6 & 23 & 13.9 & 16.2 \\
\hline PCM & 31.5 & 16.5 & 23.2 & 15.6 & 13.2 \\
\hline NCM & 32.1 & 15.3 & 21.6 & 17.4 & 13.6 \\
\hline & OR $[95 \% \mathrm{CI}]$ & Reference group & OR $[95 \% \mathrm{CI}]$ & OR $[95 \% \mathrm{CI}]$ & OR $[95 \% \mathrm{CI}]$ \\
\hline \multicolumn{6}{|c|}{ Cancer vs no cancer differences in adherence (Reference: NCW) } \\
\hline $\mathrm{BCW}$ & $1.10[1.02,1.18]^{*}$ & & $1.10[1.03,1.17]^{* *}$ & $0.97[0.88,1.07]$ & $1.00[0.90,1.10]$ \\
\hline $\mathrm{CCW}$ & $0.73[0.61,0.84]^{* * *}$ & & $0.91[0.81,1.01]$ & $0.67[0.53,0.81]^{* * *}$ & $0.88[0.74,1.03]$ \\
\hline CCM & $0.69[0.58,0.81] * * *$ & & $0.77[0.67,0.87]^{* * *}$ & $0.42[0.26,0.58] * * *$ & $0.75[0.59,0.90]^{* * *}$ \\
\hline PCM & $0.96[0.90,1.03]$ & & $0.93[0.86,0.99]^{*}$ & $0.57[0.48,0.66]^{* * *}$ & $0.73[0.63,0.82]^{* * *}$ \\
\hline NCM & $1.06[0.99,1.11]$ & & $0.93[0.89,0.98]^{* *}$ & $0.69[0.63,0.76]^{* * *}$ & $0.81[0.75,0.88]^{* * *}$ \\
\hline \multicolumn{6}{|c|}{ Cancer vs no cancer differences in adherence (Reference: NCM) } \\
\hline $\mathrm{BCW}$ & $1.03[0.95,1.11]$ & & $1.18[1.11,1.26]^{* * *}$ & $1.40[1.30,1.50]^{* * *}$ & $1.23[1.12,1.34]^{* * *}$ \\
\hline $\mathrm{CCW}$ & $0.68[0.57,0.80]^{* * *}$ & & $0.98[0.88,1.08]$ & $0.96[0.82,1.11]$ & $1.09[0.94,1.23]$ \\
\hline CCM & $0.65[0.53,0.77]^{* * *}$ & & $0.82[0.72,0.92]^{* * *}$ & $0.61[0.45,0.77]^{* * *}$ & $0.92[0.76,1.08]^{* * *}$ \\
\hline PCM & $0.91[0.84,0.98]^{* *}$ & & $0.99[0.93,1.06]$ & $0.83[0.73,0.92]^{* * *}$ & $0.90[0.80,1.00]$ \\
\hline NCW & $0.94[0.89,1.00]$ & & $1.07[1.03,1.12]^{* *}$ & $1.44[1.38,1.50] * * *$ & $1.23[1.17,1.30]^{*}$ \\
\hline \multicolumn{6}{|c|}{ Sex Differences in adherence (other categories not displayed) ${ }^{\dagger}$} \\
\hline NCW & $0.94[0.89,1.00]$ & & $1.07[1.03,1.12]^{* *}$ & $1.44[1.38,1.50]^{* * *}$ & $1.23[1.17,1.30]^{*}$ \\
\hline NCM & Reference & & & & \\
\hline CCW & $1.05[0.89,1.20]$ & & $1.19[1.05,1.32]^{*}$ & $1.58[1.37,1.78]^{* * *}$ & $1.18[0.98,1.39]$ \\
\hline CCM & Reference & & & & \\
\hline \multicolumn{6}{|c|}{ Cancer Type Differences in adherence (other categories not displayed) $)^{\ddagger}$} \\
\hline $\mathrm{BCW}$ & $1.51[1.38,1.64]^{* * *}$ & & $1.21[1.10,1.32]^{* *}$ & $1.46[1.30,1.62]^{* * *}$ & $1.13[0.97,1.30]$ \\
\hline CCW & Reference & & & & \\
\hline PCM & $1.40[1.27,1.52] * * *$ & & $1.21[1.10,1.32]^{* * *}$ & $1.35[1.18,1.53]^{* * *}$ & $0.97[0.80,1.15]$ \\
\hline CCM & Reference & & & & \\
\hline
\end{tabular}

The analysis was based on 6 repeated measures.

${ }^{\dagger}$ Other categories not displayed are: BCW and PCM. ${ }^{\ddagger}$ Other categories not displayed are: NCW and NCM. 


\section{Description of medication use and adherence}

ADH 2 Rx: Adherent to both medication classes (statins and ACEIs/ARBs/beta-blockers)

No ADH 2 Rx: Not adherent to both medication classes (reference)

ADH 1 of 2 Rx: Adherent to either statins or ACEIs/ARBs/beta-blockers

ADH 1 Rx: Use of one medication class (either statins or ACEIs/ARBs/beta-blockers) and adherent to that class

No ADH 1 Rx: Use of one medication class and not adherent to that medication class

Asterisks represent significant differences in cancer diagnosis categories, derived from GEE, which accounts for repeated observations

$* * * \mathrm{p}<0.001 ; * * 0.001 \leq \mathrm{p}<0.01 ; * 0.01 \leq \mathrm{p}<0.05$.

Abbreviations

BCW: Women with breast cancer women; CCM: Men with colorectal cancer; CCW: Women with colorectal cancer; GEE: Generalized estimating equations;

IPTW: Inverse probability of treatment weighting; NCM: Men with no cancer; NCW: Women with no cancer; PCM: Men with prostate cancer 


\subsubsection{Cancer Type and Medication Adherence}

BCW were more likely to be adherent to both $[\mathrm{OR}=1.51 ; 95 \% \mathrm{CI}=1.38,1.64 ; P<0.001]$ medication classes compared to CCW. PCM were more likely to be adherent to both medication classes compared to CCM [OR = 1.39; 95\% CI $=1.27,1.52 ; P<0.001]$. Among those with single medication use, similar patterns were observed.

Table 2.4 displays the adjusted odds ratios (AORs) and 95\% CI from the adjusted GEE regressions on adherence categories. The findings were similar to unadjusted models. For example, CCW were less likely to be adherent to both classes of medications compared to NCW [AOR $=0.70 ; 95 \%$ CI $=0.58,0.81 ; P<0.0001$. Similarly, CCM [AOR = 0.63; 95\% CI = 0.51, 0.75; $P<0.0001]$ and PCM $[$ AOR = 0.92; 95\% CI = 0.85, 0.99; $P=$ 0.022] were less likely to be adherent to both classes of medications compared to NCM.

However, there was no significant differences observed in any of the adherence categories between BCW compared to NCW, after controlling for other factors. This is in contrast to unadjusted model, in which, there was significant different between BCW as compared to NCW.

Table 2.5 displays the AORs and 95\% CI from the adjusted GEE models on adherence for other independent variables. Medicare beneficiaries who were $<80$ years, Whites, had no concordant physical health conditions, had discordant physical health conditions, had mental health conditions, had tobacco and alcohol use, and had no cardiologist visit were less likely to be adherent to both medication classes

\subsubsection{Secondary Analyses}

As CCW and CCM were generally non-adherent to medications, we further examined the relationship between cancer-related factors (stage of diagnosis and cancer treatment (surgery, chemo, radiation therapies) among men and women with CAD and incident colorectal cancer (CC) (Table 2.6). These analyses indicated that those with advanced cancer stage were less likely to be adherent to both medication classes (AOR $=0.55 ; 95 \% \mathrm{CI}=0.31,0.78)$ compared to early-stage cancer. Similarly, those who had surgery were less likely to be adherent to both medication classes $(\mathrm{AOR}=0.64 ; 95 \% \mathrm{CI}=0.45,0.82)$. 
Table 2.4. Adjusted GEE Models with IPTW on Adherence to Medication Adherence Categories

\begin{tabular}{|c|c|c|c|c|c|c|c|c|c|c|c|c|}
\hline \multirow{2}{*}{ Sex and cancer categories } & \multicolumn{3}{|c|}{ ADH 2 Rx } & \multicolumn{3}{|c|}{ ADH 1 Rx of 2} & \multicolumn{3}{|c|}{ ADH 1 Rx } & \multicolumn{3}{|c|}{ No ADH 1 Rx } \\
\hline & AOR & 95\% CI & Sig & AOR & $95 \% \mathrm{CI}$ & Sig & AOR & $95 \%$ CI & Sig & AOR & 95\% CI & Sig \\
\hline \multicolumn{13}{|c|}{ Cancer vs no cancer differences in adherence (Reference = NCW) } \\
\hline BCW & 1.08 & {$[1.00,1.17]$} & & 1.08 & {$[1.00,1.15]$} & & 1.03 & {$[0.93,1.13]$} & & 1.06 & {$[0.96,1.17]$} & \\
\hline CCW & 0.70 & {$[0.58,0.81]$} & $* * *$ & 0.87 & {$[0.77,0.97]$} & $* *$ & 0.65 & {$[0.51,0.80]$} & $* * *$ & 0.91 & {$[0.76,1.05]$} & \\
\hline CCM & 0.63 & {$[0.51,0.75]$} & $* * *$ & 0.71 & {$[0.61,0.82]$} & $* * *$ & 0.46 & {$[0.30,0.63]$} & $* * *$ & 0.85 & {$[0.70,1.01]$} & \\
\hline PCM & 0.92 & {$[0.85,0.99]$} & * & 0.89 & {$[0.82,0.95]$} & $* * *$ & 0.68 & {$[0.59,0.78]$} & $* * *$ & 0.86 & {$[0.76,0.96]$} & $* *$ \\
\hline NCM & 1.02 & {$[0.97,1.07]$} & & 0.91 & {$[0.86,0.96]$} & $* * *$ & 0.78 & {$[0.71,0.84]$} & $* * *$ & 0.89 & {$[0.82,0.96]$} & $* *$ \\
\hline NCW & Refer & & & & & & & & & & & \\
\hline \multicolumn{13}{|c|}{ Cancer vs no cancer differences in adherence (Reference = NCM) } \\
\hline BCW & 1.06 & {$[0.98,1.15]$} & & 1.18 & {$[1.11,1.26]$} & **** & 1.32 & {$[1.22,1.43]$} & $* * *$ & 1.19 & {$[1.08,1.30]$} & $* *$ \\
\hline CCW & 0.68 & {$[0.56,0.80]$} & $* * *$ & 0.96 & {$[0.86,1.07]$} & & 0.84 & {$[0.69,0.99]$} & $*$ & 1.02 & {$[0.87,1.17]$} & \\
\hline CCM & 0.62 & {$[0.49,0.74]$} & $* * *$ & 0.78 & {$[0.68,0.89]$} & $* * *$ & 0.59 & {$[0.42,0.76]$} & $* * *$ & 0.96 & {$[0.80,1.12]$} & \\
\hline PCM & 0.90 & {$[0.83,0.98]$} & $* *$ & 0.98 & {$[0.91,1.04]$} & & 0.88 & {$[0.78,0.98]$} & * & 0.96 & {$[0.86,1.07]$} & \\
\hline NCW & 0.98 & {$[0.93,1.03]$} & & 1.10 & {$[1.05,1.15]$} & $* * *$ & 1.29 & {$[1.22,1.36]$} & $* * *$ & 1.12 & {$[1.05,1.19]$} & $* *$ \\
\hline NCM & Refer & & & & & & & & & & & \\
\hline \multicolumn{13}{|c|}{ Sex Differences in adherence (other categories not displayed) ${ }^{\dagger}$} \\
\hline $\begin{array}{l}\text { NCW } \\
\text { NCM }\end{array}$ & $\begin{array}{c}0.98 \\
\text { Refer }\end{array}$ & $\begin{array}{l}{[0.93,1.03]} \\
\text { ice }\end{array}$ & & 1.10 & {$[1.05,1.15]$} & $* * *$ & 1.29 & {$[1.22,1.36]$} & $* * *$ & 1.12 & {$[1.05,1.19]$} & $* *$ \\
\hline $\begin{array}{l}\text { CCW } \\
\text { CCM }\end{array}$ & $\begin{array}{l}1.11 \\
\text { Refer }\end{array}$ & $\begin{array}{l}{[0.95,1.27]} \\
\text { ce }\end{array}$ & & 1.23 & {$[1.09,1.36]$} & $* *$ & 1.41 & {$[1.20,1.63]$} & ** & 1.06 & {$[0.85,1.27]$} & \\
\hline \multicolumn{13}{|c|}{ Cancer Type Differences in adherence (other categories not displayed) ${ }^{\ddagger}$} \\
\hline $\begin{array}{l}\text { BCW } \\
\text { CCW }\end{array}$ & $\begin{array}{l}1.55 \\
\text { Refer }\end{array}$ & $\begin{array}{l}{[1.42,1.69]} \\
\text { ce }\end{array}$ & $* * *$ & 1.23 & {$[1.11,1.35]$} & *** & 1.57 & {$[1.40,1.74]$} & $* * *$ & 1.17 & {$[1.00,1.34]$} & \\
\hline $\begin{array}{l}\text { PCM } \\
\text { CCM }\end{array}$ & $\begin{array}{c}1.46 \\
\text { Refer }\end{array}$ & $\begin{array}{l}{[1.33,1.60]} \\
\text { ce }\end{array}$ & $* * *$ & 1.24 & {$[1.13,1.36]$} & $* * *$ & 1.48 & {$[1.30,1.66]$} & $* * *$ & 1.01 & {$[0.83,1.18]$} & \\
\hline
\end{tabular}

${ }^{\dagger}$ Other categories not displayed include BCW and PCM. ${ }^{\ddagger}$ Other categories not displayed include NCW and NCM.

Description of medication use and adherence

ADH 2 Rx: Adherent to both medication classes (statins and ACEIs/ARBs/beta-blockers)

No ADH 2 Rx: Not adherent to both medication classes (reference)

ADH 1 of 2 Rx: Adherent to either statins or ACEIs/ARBs/beta-blockers

ADH 1 Rx: Use of one medication class (either statins or ACEIs/ARBs/beta-blockers) and adherent to that class

No ADH 1 Rx: Use of one medication class and not adherent to that medication class 
Asterisks represent significant differences in cancer diagnosis categories, derived from GEE, which accounts for repeated observations. Adjusted GEE model controlled for patient, physician, health-system, and environmental factors.

${ }^{* * *} \mathrm{p}<0.001 ; * * 0.001 \leq \mathrm{p}<0.01 ; * 0.01 \leq \mathrm{p}<0.05$.

\section{Abbreviations}

AOR: Adjusted odds ratio; BCW: Women with breast cancer; CAD: Coronary artery disease; CCM: Men with colorectal cancer; CCW: Women with colorectal cancer; CI: Confidence interval; GEE: Generalized estimating equations; IPTW: Inverse probability of treatment weighting; NCM: Men with no cancer; Women with no cancer; PCM: Men with prostate cancer; Sig: Significance 
Table 2.5. Adjusted GEE Models with IPTW on Medication Adherence Categories-Other factors included based on conceptual framework

\begin{tabular}{|c|c|c|c|c|c|c|c|c|c|c|c|c|}
\hline \multirow{2}{*}{ Characteristics } & \multicolumn{3}{|c|}{ ADH 2 Rx } & \multicolumn{3}{|c|}{ ADH 1 of 2 Rx } & \multicolumn{3}{|c|}{ ADH 1 Rx } & \multicolumn{3}{|c|}{ No ADH 1 Rx } \\
\hline & AOR & 95\% CI & Sig & AOR & $95 \% \mathrm{CI}$ & Sig & AOR & 95\% CI & Sig & AOR & $95 \% \mathrm{CI}$ & Sig \\
\hline \multicolumn{13}{|c|}{ Patient-level factors - Demographics } \\
\hline \multicolumn{13}{|l|}{ Age groups (in years) } \\
\hline $68-70$ & 0.94 & {$[0.88,1.00]$} & & 0.98 & {$[0.92,1.03]$} & & 0.55 & {$[0.47,0.63]$} & $* * *$ & 0.67 & {$[0.59,0.76]$} & $* * *$ \\
\hline $71-74$ & 0.90 & {$[0.84,0.96]$} & $* * *$ & 0.98 & {$[0.92,1.03]$} & & 0.58 & {$[0.50,0.65]$} & $* * *$ & 0.66 & {$[0.58,0.74]$} & $* * *$ \\
\hline $75-79$ & 0.95 & {$[0.90,1.01]$} & & 1.01 & {$[0.96,1.06]$} & & 0.68 & {$[0.61,0.75]$} & $* * *$ & 0.76 & {$[0.69,0.84]$} & $* * *$ \\
\hline$\geq 80$ & & Reference & & & - & & & - & & & - & \\
\hline \multicolumn{13}{|l|}{ Race/Ethnicity } \\
\hline African Americans & 0.63 & {$[0.55,0.72]$} & $* * *$ & 0.78 & {$[0.71,0.85]$} & $* * *$ & 0.75 & {$[0.65,0.85]$} & $* * *$ & 1.04 & {$[0.95,1.14]$} & \\
\hline Hispanics & 0.59 & {$[0.47,0.71]$} & $* * *$ & 0.74 & {$[0.63,0.84]$} & $* * *$ & 0.61 & {$[0.46,0.76]$} & $* * *$ & 0.85 & {$[0.71,1.00]$} & $*$ \\
\hline Others & 0.90 & {$[0.83,0.98]$} & $*$ & 0.87 & {$[0.80,0.94]$} & $* * *$ & 0.83 & {$[0.73,0.93]$} & $* * *$ & 0.89 & {$[0.79,1.00]$} & $*$ \\
\hline Whites & & Reference & & & & & & & & & & \\
\hline \multicolumn{13}{|c|}{ Patient-level factors - Census-tract socioeconomic status } \\
\hline \multicolumn{13}{|l|}{ Education level } \\
\hline$<12 \%$ (national avg) & 1.01 & {$[0.96,1.07]$} & & 0.99 & {$[0.95,1.04]$} & & 1.00 & {$[0.94,1.07]$} & & 1.05 & {$[0.98,1.11]$} & \\
\hline$\geq 12 \%$ (national avg) & & Reference & & & - & & & - & & & - & \\
\hline \multicolumn{13}{|l|}{ Poverty level } \\
\hline$<14.3 \%$ (national avg) & 1.03 & {$[0.98,1.08]$} & & 1.06 & {$[1.02,1.11]$} & $* *$ & 1.11 & {$[1.04,1.17]$} & $* *$ & 1.10 & {$[1.04,1.16]$} & $* *$ \\
\hline$\geq 14.3 \%$ (national avg) & & Reference & & & - & & & - & & & - & \\
\hline \multicolumn{13}{|c|}{ Patient-level factors - Chronic physical and mental health conditions } \\
\hline \multicolumn{13}{|l|}{ Concordant PHCs } \\
\hline Yes & 1.40 & {$[1.29,1.50]$} & $* * *$ & 1.40 & {$[1.31,1.50]$} & $* * *$ & 0.70 & {$[0.59,0.81]$} & $* * *$ & 0.48 & {$[0.37,0.60]$} & $* * *$ \\
\hline No & & Reference & & & - & & & - & & & - & \\
\hline \multicolumn{13}{|l|}{ Discordant PHCs } \\
\hline Yes & 0.78 & {$[0.73,0.83]$} & $* * *$ & 0.88 & {$[0.84,0.92]$} & $* * *$ & 1.12 & {$[1.06,1.17]$} & $* * *$ & 1.27 & {$[1.22,1.33]$} & $* * *$ \\
\hline No & & Reference & & & - & & & - & & & - & \\
\hline \multicolumn{13}{|l|}{ Mental health conditions } \\
\hline Yes & 0.66 & {$[0.60,0.71]$} & $* * *$ & 0.77 & {$[0.72,0.82]$} & $* * *$ & 0.88 & {$[0.82,0.95]$} & $* * *$ & 1.08 & {$[1.02,1.15]$} & $*$ \\
\hline No & & Reference & & & - & & & - & & & - & \\
\hline CAD severity & 1.02 & {$[0.91,1.13]$} & & 1.05 & {$[0.95,1.15]$} & & 0.57 & {$[0.42,0.71]$} & $* * *$ & 0.63 & {$[0.49,0.78]$} & $* * *$ \\
\hline \multicolumn{13}{|c|}{ Patient-level factors - Personal health behavior } \\
\hline \multicolumn{13}{|l|}{ Tobacco use } \\
\hline Yes & 1.32 & {$[1.21,1.43]$} & $* * *$ & 1.22 & {$[1.13,1.31]$} & $* * *$ & 1.44 & {$[1.30,1.58]$} & $* * *$ & 1.14 & {$[1.01,1.28]$} & \\
\hline No & & Reference & & & - & & & - & & & - & \\
\hline \multicolumn{13}{|l|}{ Alcohol use } \\
\hline Yes & 1.48 & {$[1.27,1.69]$} & $* * *$ & 1.28 & {$[1.10,1.46]$} & $* *$ & 1.00 & {$[0.74,1.26]$} & & 0.63 & {$[0.39,0.87]$} & $* * *$ \\
\hline No & & Reference & & & - & & & - & & & - & \\
\hline
\end{tabular}




\begin{tabular}{|c|c|c|c|c|c|c|c|c|c|c|c|c|}
\hline \multirow{2}{*}{ Characteristics } & \multicolumn{3}{|c|}{ ADH 2 Rx } & \multicolumn{3}{|c|}{ ADH 1 of 2 Rx } & \multicolumn{3}{|c|}{ ADH 1 Rx } & \multicolumn{3}{|c|}{ No ADH 1 Rx } \\
\hline & AOR & $95 \% \mathrm{CI}$ & Sig & AOR & $95 \% \mathrm{CI}$ & Sig & AOR & $95 \% \mathrm{CI}$ & Sig & AOR & $95 \% \mathrm{CI}$ & Sig \\
\hline \multicolumn{13}{|c|}{ Health System Factors } \\
\hline \multicolumn{13}{|l|}{ Donut hole } \\
\hline Exceeded & 0.96 & {$[0.87,1.05]$} & & 0.99 & {$[0.89,1.09]$} & & 1.00 & {$[0.91,1.10]$} & & 0.99 & {$[0.88,1.10]$} & \\
\hline Not reached & 0.35 & {$[0.26,0.43]$} & $* * *$ & 0.59 & {$[0.51,0.68]$} & $* * *$ & 0.66 & {$[0.56,0.77]$} & $* * *$ & 1.32 & {$[1.20,1.44]$} & $* * *$ \\
\hline Reached & & Reference & & & $\begin{array}{c}- \\
-\end{array}$ & & & $\begin{array}{c}20.001 \\
-\end{array}$ & & & - & \\
\hline \multicolumn{13}{|c|}{ Provider Related Factors } \\
\hline \multicolumn{13}{|l|}{ Primary care visit } \\
\hline Yes & 1.01 & {$[0.97,1.05]$} & & 1.08 & {$[1.04,1.11]$} & $* * *$ & 0.95 & {$[0.91,1.00]$} & & 0.96 & {$[0.91,1.01]$} & \\
\hline No & & Reference & & & - & & & - & & & - & \\
\hline \multicolumn{13}{|l|}{ Cardiologist visit } \\
\hline Yes & 1.04 & {$[1.01,1.07]$} & $*$ & 1.12 & {$[1.09,1.15]$} & $* * *$ & 0.69 & {$[0.65,0.73]$} & $* * *$ & 0.70 & {$[0.66,0.74]$} & $* * *$ \\
\hline No & & Reference & & & - & & & - & & & - & \\
\hline \multicolumn{13}{|c|}{ External Environment and County-level Factors } \\
\hline \multicolumn{13}{|l|}{ SEER Region } \\
\hline Northeast & 1.07 & {$[1.00,1.15]$} & & 1.05 & {$[0.99,1.12]$} & & 1.07 & {$[0.97,1.16]$} & & 0.94 & {$[0.84,1.03]$} & \\
\hline South & 0.96 & {$[0.89,1.04]$} & & 1.05 & {$[0.99,1.12]$} & & 0.98 & {$[0.88,1.07]$} & & 1.02 & {$[0.92,1.12]$} & \\
\hline North-central & 1.17 & {$[1.09,1.25]$} & $* * *$ & 1.08 & {$[1.01,1.15]$} & * & 1.14 & {$[1.04,1.24]$} & $* *$ & 0.94 & {$[0.84,1.05]$} & \\
\hline West & & Reference & & & - & & & - & & & - & \\
\hline \multicolumn{13}{|l|}{ Metropolitan status } \\
\hline Metro & 0.92 & {$[0.84,0.99]$} & * & 0.94 & {$[0.87,1.00]$} & * & 0.89 & {$[0.80,0.98]$} & * & 1.00 & {$[0.91,1.10]$} & \\
\hline Non-metro & & Reference & & & - & & & - & & & - & \\
\hline \multicolumn{13}{|l|}{ HPSA } \\
\hline No shortage & 1.07 & {$[0.98,1.15]$} & & 1.04 & {$[0.96,1.12]$} & & 1.03 & {$[0.92,1.13]$} & & 0.95 & {$[0.83,1.06]$} & \\
\hline Part county & 1.07 & {$[1.01,1.12]$} & $*$ & 1.03 & {$[0.99,1.08]$} & & 1.02 & {$[0.96,1.09]$} & & 1.04 & {$[0.97,1.11]$} & \\
\hline Whole county & & Reference & & & - & & & - & & & - & \\
\hline \multicolumn{13}{|l|}{ FQHC } \\
\hline None & 1.15 & {$[1.05,1.26]$} & $* *$ & 1.04 & {$[0.95,1.13]$} & & 1.14 & {$[1.01,1.26]$} & & 1.03 & {$[0.90,1.16]$} & \\
\hline 1 to 10 & 1.05 & {$[0.98,1.11]$} & & 0.99 & {$[0.94,1.05]$} & & 1.10 & {$[1.02,1.18]$} & * & 1.05 & {$[0.97,1.14]$} & \\
\hline \multirow{2}{*}{\multicolumn{13}{|c|}{ Rural health centers }} \\
\hline & & & & & & & & & & & & \\
\hline Yes & 1.02 & {$[0.97,1.08]$} & & 0.99 & {$[0.94,1.05]$} & & 1.02 & {$[0.95,1.09]$} & & 1.03 & {$[0.96,1.10]$} & \\
\hline No & & Reference & & & - & & & - & & & - & \\
\hline \% cardiologists in county & 0.84 & {$[0.75,0.93]$} & $* * *$ & 0.92 & {$[0.84,1.00]$} & * & 1.08 & {$[0.96,1.19]$} & & 1.14 & {$[1.02,1.25]$} & $*$ \\
\hline$\%$ oncologists in county & 2.23 & {$[1.79,2.68]$} & $* * *$ & 1.37 & {$[0.99,1.76]$} & & 0.68 & {$[0.13,1.24]$} & & 0.55 & {$[0.02,1.12]$} & $*$ \\
\hline Time & 1.41 & {$[1.40,1.41]$} & $* * *$ & 1.26 & {$[1.25,1.26]$} & $* * *$ & 1.35 & {$[1.35,1.36]$} & $* * *$ & 1.12 & {$[1.12,1.13]$} & $* * *$ \\
\hline
\end{tabular}


Time-invariant characteristics include cancer diagnosis categories, age, race/ethnicity, poverty status, high school education, concordant and discordant physical health conditions, SMI, SEER region, and county-level characteristics. Time-variant characteristics include, tobacco use, alcohol use, depression, anxiety, and routine follow-up with primary care physician and/or cardiologist, and donut hole.

Concordant physical health conditions consisted of diabetes, hyperlipidemia, hypertension, stroke, cardiac arrhythmia, and congestive heart failure. Discordant physical health conditions consisted of arthritis, asthma, chronic obstructive pulmonary disease, osteoporosis, dementia, human immunodeficiency virus, and hepatitis. Mental health conditions include anxiety, depression, SMI (schizophrenia, bipolar disorder, and psychoses)

\section{Description of medication use and adherence}

ADH 2 Rx: Adherent to both medication classes (statins and ACEIs/ARBs/beta-blockers)

No ADH 2 Rx: Not adherent to both medication classes (reference)

ADH 1 of 2 Rx: Adherent to either statins or ACEIs/ARBs/beta-blockers

ADH 1 Rx: Use of one medication class (either statins or ACEIs/ARBs/beta-blockers) and adherent to that class

No ADH 1 Rx: Use of one medication class and not adherent to that medication class

Asterisks represent significant differences in cancer diagnosis categories, derived from GEE, which accounts for repeated observations.

${ }^{* * *} \mathrm{p}<0.001 ; * * 0.001 \leq \mathrm{p}<0.01 ; * 0.01 \leq \mathrm{p}<0.05$.

\section{Abbreviations}

AOR: Adjusted odds ratio; CI: Confidence interval; FQHC: Federally qualified health centers; GEE: Generalized estimating equations; HPSA: Health professional shortage area; IPTW: inverse probability treatment weights; PHC: Physical health condition; Sig: Significance; SMI: Severe mental illness 
Table 2.6. Adjusted GEE models on Medication Adherence Categories among Patients with Colorectal Cancer (N = 3,104)

\begin{tabular}{|c|c|c|c|c|c|c|c|c|c|c|c|c|}
\hline \multirow{2}{*}{ Characteristics } & \multicolumn{3}{|c|}{ ADH 2 Rx } & \multicolumn{3}{|c|}{ ADH 1 of 2 Rx } & \multicolumn{3}{|c|}{ ADH 1 Rx } & \multicolumn{3}{|c|}{ No ADH 1 Rx } \\
\hline & AOR & $95 \%$ CI & Sig & AOR & $95 \% \mathrm{CI}$ & Sig & AOR & $95 \% \mathrm{CI}$ & Sig & AOR & $95 \%$ CI & Sig \\
\hline \multicolumn{13}{|l|}{ Cancer stage } \\
\hline Stage II & 0.77 & {$[0.54,0.99]$} & $*$ & 0.82 & {$[0.61,1.02]$} & & 1.09 & {$[0.82,1.35]$} & & 1.21 & {$[0.94,1.48]$} & \\
\hline Stage III/IV & 0.55 & {$[0.31,0.78]$} & $* * *$ & 0.63 & {$[0.41,0.84]$} & $* * *$ & 0.72 & {$[0.43,1.00]$} & $*$ & 0.84 & {$[0.56,1.13]$} & \\
\hline Stage $0 / \mathrm{I}$ & & Reference & & & & & & & & & & \\
\hline \multicolumn{13}{|l|}{ Surgery } \\
\hline Yes & 0.64 & {$[0.45,0.82]$} & $* * *$ & 0.72 & {$[0.53,0.91]$} & $* * *$ & 0.66 & {$[0.47,0.85]$} & $* * *$ & 0.96 & {$[0.76,1.16]$} & \\
\hline No & & Reference & & & & & & & & & & \\
\hline \multicolumn{13}{|l|}{ Chemotherapy } \\
\hline Yes & 1.03 & {$[0.77,1.28]$} & & 1.25 & {$[1.02,1.49]$} & & 0.97 & {$[0.66,1.27]$} & & 1.32 & {$[1.03,1.62]$} & \\
\hline No & & Reference & & & & & & & & & & \\
\hline \multicolumn{13}{|c|}{ Radiation therapy } \\
\hline Yes & 0.91 & {$[0.54,1.29]$} & & 0.89 & {$[0.52,1.26]$} & & 0.74 & {$[0.28,1.19]$} & & 0.82 & {$[0.38,1.26]$} & \\
\hline No & & Reference & & & & & & & & & & \\
\hline \multicolumn{13}{|l|}{ Hormone therapy } \\
\hline Yes & 0.87 & {$[0.28,1.42]$} & & 0.52 & {$[0.32,1.55]$} & & 1.24 & {$[0.80,1.55]$} & & 0.42 & {$[0.23,1.57]$} & \\
\hline No & & Reference & & & & & & & & & & \\
\hline
\end{tabular}

Description of medication use and adherence

ADH 2 Rx: Adherent to both medication classes (statins and ACEIs/ARBs/beta-blockers)

No ADH 2 Rx: Not adherent to both medication classes (reference)

ADH 1 of 2 Rx: Adherent to either statins or ACEIs/ARBs/beta-blockers

ADH 1 Rx: Use of one medication class (either statins or ACEIs/ARBs/beta-blockers) and adherent to that class

No ADH 1 Rx: Use of one medication class and not adherent to that medication class

Asterisks represent significant differences in cancer diagnosis categories, derived from GEE models, which controlled for patient, physician, health-system, and environmental factors.

$* * * \mathrm{p}<0.001 ; * * 0.001 \leq \mathrm{p}<0.01 ; * 0.01 \leq \mathrm{p}<0.05$.

Abbreviations

AOR: Adjusted odds ratio; CI: Confidence interval; GEE: Generalized estimating equations; Sig: Significance 


\subsection{Discussion}

The current study analyzed the relationship between incident cancer diagnosis and adherence to statins and/or ACEIs/ARBs/beta-blockers among elderly FFS Medicare beneficiaries with pre-existing CAD. To the best of our knowledge, this is the first study to compare medication adherence to statins and/or ACEIs/ARBs/beta-blockers between cancer and non-cancer cohorts with CAD. In general, only $28.9 \%$ of the study population were adherent to both statins and ACEIs/ARBs/beta blockers. Although there are no published studies comparing cancer and noncancer patients, our figures are similar to existing studies on medication adherence to multiple medication classes in those with cardiovascular conditions. Given that there is dearth of studies in the area of multiple medication adherence among those with CAD, specifically among those with elderly, it is important to learn from published studies in cardiovascular conditions. One study showed that $21.4 \%$ adults with cardiovascular conditions were adherent to both statins and calcium channel blockers [159]. Similarly, another study reported that 21\% had combined adherence on beta-blockers, lipid lowering therapy, and aspirin [160].

These findings emphasize the need for multimodal interventions for improving medication adherence. Ho et al reviewed studies on medication adherence among individuals with cardiovascular health conditions and suggested that multimodal interventions including the pharmacist-led interventions may be successful in realizing the benefits of medication adherence and reducing the costs of non-adherence [141]. Future studies need to examine whether the proven strategies of medication adherence can also be applied to improve medication adherence of multiple drug regimens.

We observed that both men and women diagnosed with incident colorectal cancer were less likely to adhere to both statins and ACEIs/ARBs/beta-blockers compared to non-cancer women and men, even after controlling for a comprehensive list of factors. Similarly, men with incident prostate cancer were less likely to adhere to both medication classes, but the magnitude of difference was much less compared to men with no cancer diagnosis. There were no differences in adherence to both medication classes by presence of breast cancer. Taken together, these finding suggest that cancer type rather than cancer itself may affect medication adherence. Furthermore, we also found that among Medicare beneficiaries with CAD and colorectal cancer, those with late-stage cancer compared to early stage cancer and those who had surgical treatment for cancer compared to no surgical treatment were less likely to be adherent to both statins and ACEIs/ARBs/beta blockers. These findings suggest that cancer stage and cancer treatment may affect medication adherence among colorectal cancer patients. It is also plausible that the lifethreatening nature of CC may lead to prioritization of cancer care. For example, colorectal cancer has lower 5-year survival rates compared to breast and prostate cancer (66\% vs. 91\% and 99\%, respectively) [106].

Despite the complexity of care among those with multiple chronic conditions (in our case those with CAD and $\mathrm{CC}$ ), it is important to focus on medication adherence for CAD. However, under the fee-for-service programs, there are no incentives to coordinate care across multiple systems, providers, and payers [141]. In this context, the new fiscal incentives by the CMS in paying physicians who provide care for patients with multiple chronic conditions and who invest time and resources to provide more coordinated and patient-centered care [161] may also promote adherence. It has to be noted that a systematic review of medication adherence among those with multiple chronic conditions reported that medication adherence interventions were "almost exclusively" conducted by the 
pharmacists [162]. In recognition of the critical role of pharmacists, Medicare now covers Medication Management Services (MTM) of pharmacists. There is some evidence that such policies (i.e. MTM services offered by pharmacists) can improve adherence and lead to better cardiovascular outcomes [163].

\subsubsection{Clinical Implications}

Our study findings have implications for clinical practice. As mentioned in the introduction, statins and ACEIs/ARBs/beta-blockers are central to improving CAD-related health outcomes and statins have been shown to reduce cancer-specific mortality in those with breast, colorectal, and prostate cancer [144-146, 164]. Furthermore, medications for CAD may also reduce cancer treatment-related cardiotoxicity [71]. We do recognize that medication adherence is a multidimensional issue in which providers, patients, payers, and policy makers are inextricably linked and all of them need to be involved in improving medication adherence [165]. It may be very challenging for clinicians to implement interventions in routine practice because successful to date have included multiple, heterogeneous components [141]. In this context, the recent healthcare delivery models such as the medical home may help in overcoming the challenges to coordination across different providers. It has been shown that patientcentered medical homes can improve adherence to medications among those with cardiovascular conditions [166].

\subsubsection{Strengths and Limitations}

Our study findings need to be interpreted in the context of its limitations. The study findings cannot be generalized to all Medicare beneficiaries because the study population is restricted to those residing in SEER regions and to those with FFS Medicare plans. Although we used a proxy measure for CAD severity, this measure may not accurately capture severity of the disease. We were not able to control for patient-level lifestyle health behaviors, knowledge, attitude, and preferences as well as number of cancer-related complications, treatment side-effects, and cost-related factors that may affect adherence. We used prescription claims for measuring adherence. As part D file contains only filled prescriptions, it is not known whether the patient actually used the medications or whether the patients they adhered to their providers' instructions or not. The focus of the study was only on statins and ACEIs/ARBs/beta-blockers, as they are recommended by guidelines for CAD long-term therapy. Substitution with any other drugs other than those included in the study are considered non-users of the respective medication. This does not affect adherence as those drugs are not recommended by guidelines for long-term CAD pharmacotherapy.

Our study has many advantages. The study used a rigorous study design comparing cancer and non-cancer cohort over time and statistical adjustment for selection bias in observed characteristics. Medicare FFS claims data enabled us to measure adherence over time and followed a large cohort of patients for a long period of time across a variety of providers. The study adjusted a comprehensive list of factors that can influence medication adherence. The study included individuals with significant medical comorbidities, typically excluded from randomized clinical trials and examined real-world practice patterns.

\subsubsection{Conclusions}


The findings from our study contribute to existing literature on adherence and adds to the nascent field of cardio-oncology. We observed that medication adherence was associated with cancer type rather than just the presence of cancer itself. Elderly Medicare beneficiaries with colorectal cancer and to a lesser extent men with prostate cancer were less likely to be adherent to evidence-based medications for CAD. These findings highlight the vulnerability of these individuals and the difficulty in sustaining ongoing therapy for CAD. The recent healthcare delivery models such as the medical home may help in overcoming the challenges to coordination across different providers and can improve adherence to medications among those with cardiovascular conditions. 


\section{CHAPTER 3}

\section{Non-adherence to Statins and Antihypertensive Medications and Hospitalizations among Elderly Fee-for-Service Medicare Beneficiaries with Pre-existing Coronary Artery Disease and Incident Cancer}

\subsection{Abstract}

Objectives: To examine the relationship between adherence to both statins and ACEIs/ARBs/beta-blockers and coronary artery disease (CAD)-related hospitalizations.

Methods: A retrospective observational longitudinal study was conducted using SEER-Medicare data. Elderly Medicare fee-for-service beneficiaries with pre-existing CAD and incident breast, colorectal, or prostate cancer $(\mathrm{N}=$ 12,096) were observed for a period of 12 months before and 12 months after cancer diagnosis. Hospitalizations measured every 120-days were categorized into three groups: (1) any CAD-related hospitalization; (2) other hospitalizations; or (3) no hospitalization. Medication adherence was categorized into five mutually exclusive groups: (1) adherent to both statins and ACEIs/ARBs/beta-blockers (reference group); (2) not adherent to both statins and ACEIs/ARBs/beta-blockers; (3) adherent to either statins or ACEIs/ARBs/beta-blockers; (4) use of one medication class and adherent to that class; or (5) use of one medication class and not adherent to that medication class. The unadjusted and adjusted relationship between medication adherence and hospitalization was analyzed using repeated measures multinomial logistic regressions. Inverse probability treatment weights were used to control for observed group differences among medication adherence categories.

Results: Adherence to both statins and ACEIs/ARBs/beta-blockers was estimated at 31.2\% during the 120-day period immediately after cancer diagnosis; $13.7 \%$ were not adherent to both medication classes during the same period; 27.4\% had CAD-related hospitalizations immediately after cancer diagnosis and this percentage declined to $10.6 \%$ during the last four months of the post-cancer period. In the adjusted analyses, those not adherent to both statins and ACEIs/ARBs/beta-blockers were more likely to have CAD-related hospitalization compared to those who were adherent to both medication classes [AOR $=1.82 ; 95 \% \mathrm{CI}=1.72,1.92 ; P<0.0001$ ].

Conclusions: Given the complexity of interaction between CAD and cancer, it is important to routinely monitor medication adherence in general clinical practice and provide linkages to support services that can increase medication adherence. 


\subsection{Introduction}

Coronary artery disease (CAD) remains the leading cause of hospitalization in the United States (US), although the rate of hospitalization for CAD has declined from 77\% in 2000 to $44 \%$ in 2010 [167]. According to the Healthcare Cost and Utilization Project (HCUP), in 2010, CAD accounted for nearly 512,000 hospital admissions with an estimated inpatient expenditures of nearly $\$ 9.5$ billion [168]. Among individuals with CAD, per-capita hospital expenditures were estimated at \$22,700 in 2014 accounting for about $62 \%$ of total direct medical care expenditures $[4,169]$, suggesting that hospitalizations are the primary drivers of total direct medical care expenditures.

Existing evidence also suggests that adults, especially older individuals (age $>65$ years) with incident cancer may be at risk for non-cancer related hospitalizations. Although not specific to CAD-related hospitalizations, men with incident prostate cancer and cardio-metabolic conditions were more likely to experience hospitalization during the immediate period after cancer diagnosis [170]. It has been reported that the risk of first hospitalization for CAD increases during the six months after cancer diagnosis [171], perhaps due to cardiotoxicity associated with cancer treatments such as radiotherapy, chemotherapy, and hormone therapy [60-62]. Some studies have reported increased risk of cardiovascular events, complications, and mortality in women who were treated with radiotherapy [172-174] or were exposed to cardiotoxic drugs (trastazumab and anthracycline) [108, 175, 176]. Similarly, endocrine treatment-related cardiotoxicity has also been reported in prostate cancer patients [177-179].

Adherence to angiotensin-converting enzyme inhibitors (ACEIs), angiotensin II receptor blockers (ARBs), betablockers, and statins have been shown to not only improve survival $[95,131]$ but also reduce the risk of CADrelated hospitalizations [94, 95]. Specifically, statins have been reported to reduce cancer-specific mortality in those with breast, colorectal, and prostate cancer [144-146, 164], suggesting that adherence to statins may be even more important for individuals with cancer.

However, to date, no study has examined the relationship between adherence to statins and ACEIs/ARBs/betablockers, and CAD-related hospitalization in older individuals (age > 65 years) with pre-existing CAD and incident cancer. As old age is a risk factor for CAD-related hospitalization [168] and CAD is the most common pre-existing condition among older adults diagnosed with cancer [85, 86, 90], it is important to analyze the relationship between adherence to both statins and ACEIs/ARBs/beta-blockers and CAD-related hospitalizations among cancer patients. Therefore, the present study evaluated the relationship between adherence to both statins and ACEIs/ARBs/betablockers and CAD-related hospitalizations among elderly fee-for-service Medicare beneficiaries with pre-existing $\mathrm{CAD}$ and incident breast, colorectal or prostate cancer, after controlling for cancer treatment that are cardiotoxic and other risk factors that may affect CAD-related hospitalizations.

\subsection{Methods}

\subsubsection{Conceptual Framework}

This study adapted Andersen's Behavioral Model of health service utilization to select independent variables that may influence CAD-related hospitalizations (Figure 1.2) [124, 125]. This model categorizes variables that may influence CAD-related hospitalization into predisposing (age, sex, race), enabling (census tract education level and 
poverty status, marital status, Medicare prescription drug coverage), need factors (chronic physical and mental health conditions, cancer type, cancer stage, cancer treatment), personal health practices (tobacco and alcohol use), healthcare use (visits to primary care/cardiologist and adherence to statins and/or ACEIs/ARBs/beta-blockers) and external environment (Surveillance, Epidemiology and End Results [SEER] region and county metro status).

\subsubsection{Study Design}

The study utilized a retrospective observational longitudinal cohort design with baseline (24-36 months before cancer diagnosis), pre-cancer (12 months before cancer diagnosis) and post-cancer (12 months after cancer diagnosis) periods (Figure 3.1).

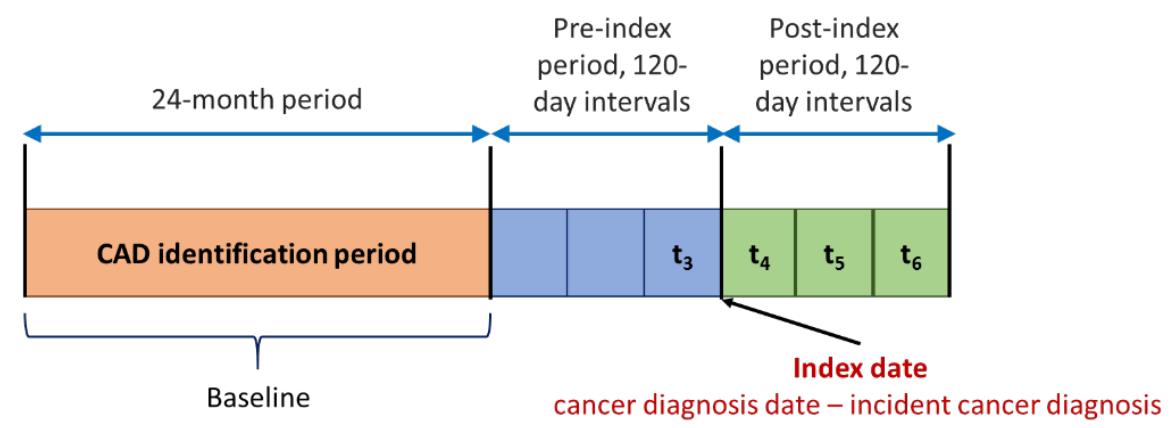

Figure 3.1. Schematic presentation of study design

\subsubsection{Data Sources}

We linked data from four sources: the SEER registries, Medicare claims, the American Community Survey (ACS), and the Area Health Resource Files (AHRF). SEER program is an epidemiologic surveillance system consisting of population-based tumor registries that collects data on all incident cases of cancer that occur in persons residing in 18 SEER areas. SEER data also consists of information about demographic characteristics of the patient, diagnosis date, cancer site, stage, and grade [126]. The ACS census tract files provided information on the census tract poverty status [128]. The AHRF was used to derive percent cardiologists and oncologists in the county [129].

\subsubsection{Study Population}

Individuals with CAD were identified using a validated algorithm developed by the Centers for Medicare and Medicaid Services (CMS) [150]. The algorithm used the International Classifications of Diseases $-9^{\text {th }}$ edition, Clinical Modification (ICD-9-CM) codes. Individuals with at least one inpatient or two outpatient claims (institutional outpatient, physician office, and home health agency claims) with a primary or secondary diagnosis of CAD during the baseline were classified as having pre-existing CAD. Individuals with incident cancer, were identified from the SEER registry for the period between January 2008 and December 2011.

Other inclusion criteria were: (1) age 68 years or older at cancer diagnosis; (2) no missing data for type and stage of cancer; (3) alive with continuous FFS Medicare Part A, B enrollment during the entire study period; (4) continuous part D enrollment during the pre- and post-index period; (5) no missing information on county, and (6) having two or more prescriptions for either statins or two or more prescriptions for any one of the following 
medication types: ACEIs, ARBs, or beta-blockers. Those who were diagnosed with cancer through autopsy or death certificate were also excluded. The selection criteria are presented in Figure 3.2

\subsubsection{Measures}

\subsubsection{Dependent Variable: Any Hospitalization for CAD Events}

The dependent variable was categorized into three mutually exclusive groups based on a hierarchy: (1) any CAD-related hospitalization; (2) other hospitalization (hospitalizations other than CAD); and (3) no hospitalization. CAD-related hospitalizations were defined as inpatient admissions for any cardiovascular event (heart attack, heart failure, angina, or stroke) and were identified from primary and secondary diagnoses using ICD-9-CM codes [180]. This measure was calculated during 120-days before cancer-diagnosis and every 120-days during the post-cancer period. A 120-day interval before cancer diagnosis was a baseline measurement to ensure that the change in hospitalization was associated with adherence and not due to any other factors.

\subsubsection{Key Independent Variable: Adherence to Statins, ACEIs/ARB/Beta-blockers}

This measure was also calculated for 120-days before cancer-diagnosis and every 120-days during the postcancer period. A 120-day interval before cancer diagnosis was a baseline measurement to account for change in adherence due to cancer diagnosis. Based on the type of drug regimen and individuals were classified into five mutually exclusive groups: (1) adherent to both statins and ACEIs/ARBs/beta-blockers; (2) not adherent to both statins and ACEIs/ARBs/beta blockers; (3) adherent to either statins or ACEIs/ARBs/beta-blockers; (4) use of one medication class and adherent to that class; and (5) use of one medication class and not adherent to that medication class.

Statins, ACEIs, ARBs, and beta-blockers were identified using the generic names in the Part $\mathrm{D}$ event files. Proportion of days covered (PDC) was used to measure adherence to statins, ACEIs, ARBs, or beta-blockers and was calculated for those who filled at least two prescriptions for either statins or two prescriptions for ACEIs, ARBs, or beta-blockers. The PDC was calculated as: $\frac{\text { Days of medication supplied during each time period }\left(t_{0}, t_{1}, t_{2}, t_{3}\right)}{\text { Days in each time period (120 days })}$. For those with a drug regimen (i.e. statins and ACEIs, ARBs or beta-blockers), PDC includes days within a specific period when an individual is covered for both statins and beta-blockers, ACEIs, or ARBs. PDC was dichotomized, where individuals with PDC $\geq 80 \%$ were considered as adherent and those with $\mathrm{PDC}<80 \%$ as non-adherent [153]. 


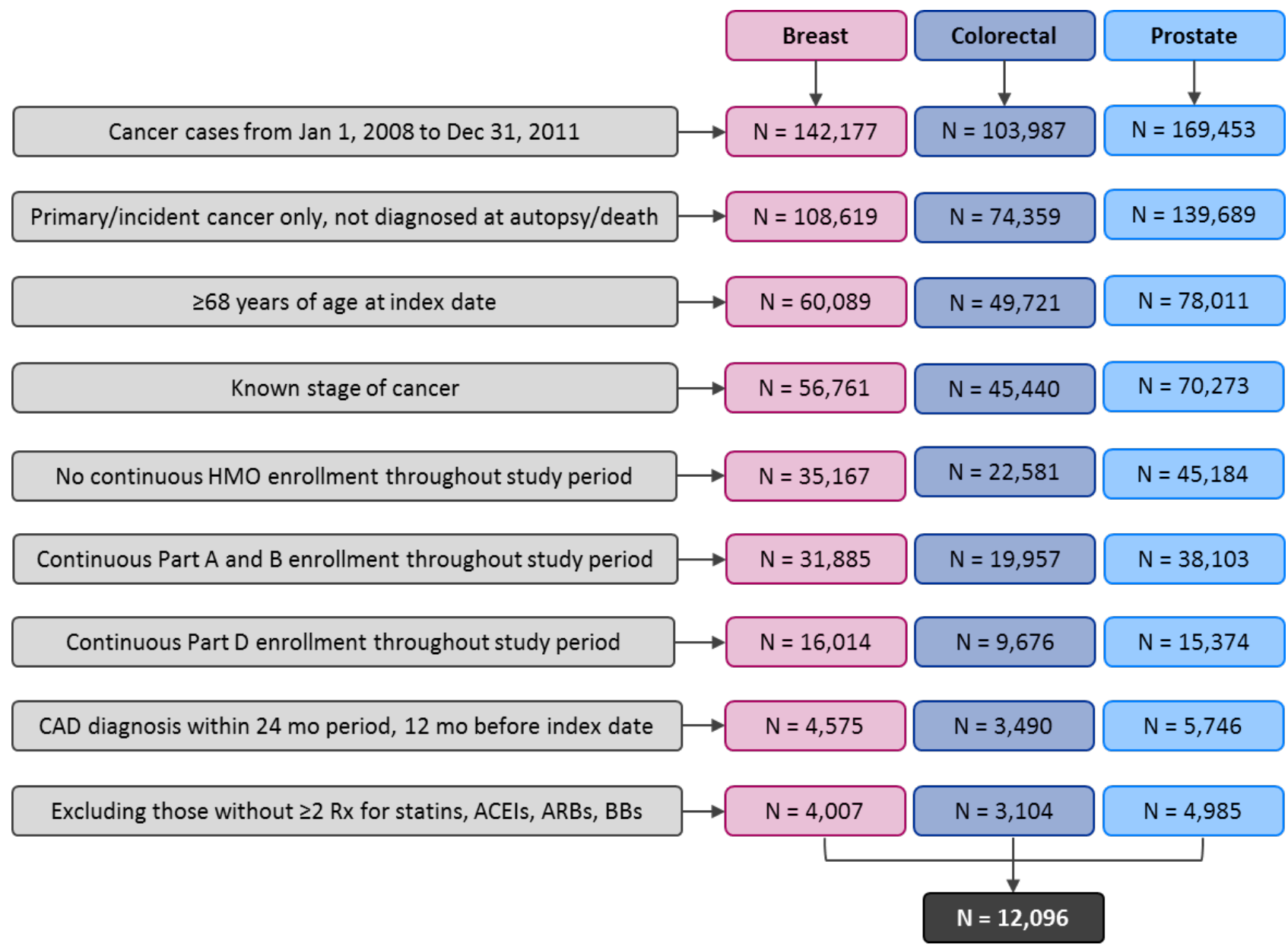

Figure 3.2. Schematic presentation of selection criteria for study cohort.

Abbreviations - ACEIs: Angiotensin-converting enzyme inhibitors; ARBs: Angiotensin II receptor blockers; BB: Beta-blockers; CAD: Coronary artery disease; HMO: Health Maintenance Organization; $\mathrm{R}_{\mathrm{x}}$ : Prescription 


\subsubsection{Other Independent Variables}

\section{Predisposing Factors}

These were time-invariant and comprised age in years measured at index month of incident cancer diagnosis, sex, and race/ethnicity.

\section{Enabling Factors}

These factors included marital status (measured at index month of incident cancer diagnosis), census tract-level education level and poverty status (measured at baseline), and Medicare Part D coverage gap (measured every 120 days). Medicare Part D coverage gap or "donut hole" is the phase between the annual coverage limit and catastrophic coverage. The Part D plans typically starts with an annual deductible, after which Medicare pays for the prescription drugs up to an initial coverage limit with certain coinsurance associated with those payments. The coverage is terminated once an individual reaches the annual coverage limit until the cost of prescription drugs reaches to catastrophic limit [156].

\section{Need-based Factors}

The cancer-related factors comprised cancer diagnosis categories, stage of cancer, and cancer treatment, measured at incident cancer diagnosis. Cancer diagnosis was categorized based on cancer type and sex because the study included sex-specific cancers (breast and prostate cancer). This variable was categorized into four groups to distinguish cancer type in relation to sex, women with breast cancer, women with colorectal cancer, men with prostate cancer, and men with colorectal cancer. Stage of cancer was defined using American Joint Committee on Cancer (AJCC) classification, categorized from stage 0 to IV [181]. Cancer treatment was categorized into three groups based on cancer site, receipt of and type of initial cancer treatment (during first four months of cancer diagnosis), and cardiotoxic nature of treatment [63-67] - (a) cardiotoxic cancer treatment includes chemotherapy, hormone therapy, and/or radiotherapy for breast cancer, chemotherapy and/or hormone therapy for prostate cancer, and chemotherapy for colorectal cancer, (b) non-cardiotoxic cancer treatment includes radiation therapy for colorectal and prostate cancer and cancer surgery (all three cancers), and (c) no cancer treatment.

For CAD severity, a proxy measure was used, constructed based on the CMS hierarchical condition category (HCC) classification system. Based on the CMS risk adjustment model, each of the HCC codes for CAD was assigned specific score based on the risk and severity [154], ranging from 0.231 to 0.349 , where higher scores represented severe manifestations of CAD.

Other physical and mental health conditions were selected using the Multiple Chronic Conditions working group chronic conditions grouping [155]. Physical chronic conditions, measured at baseline, were categorized into concordant (cardiac arrhythmias, congestive heart failure, diabetes, hyperlipidemia, hypertension, and stroke) and discordant (dementia, asthma, chronic obstructive pulmonary disease, arthritis, hepatitis, human immunodeficiency virus, and osteoporosis) conditions. Mental health conditions comprised severe mental illness (measured at baseline) and anxiety and depression (measured every 120 days). 


\section{Healthcare utilization}

Healthcare-seeking behavior was defined as primary care and cardiologist visits, measured every 120 days.

\section{Personal Health Care Practices}

This included tobacco and alcohol use as these practices negatively impact cardiovascular health. These variables were measured every 120 days.

\section{External Environment Factors}

This domain included SEER region - Northeast (Connecticut, New Jersey), South (Kentucky, Louisiana, Atlanta, rural Georgia, and Greater Georgia), North-central (Detroit, Iowa), and West (San Francisco, Hawaii, New Mexico, Seattle, Utah, San Jose, Los Angeles, Greater California), county metro status (based on urban/rural continuum codes from the AHRF), and percentage of cardiologists and oncologists in the county). These were timeinvariant and measured at baseline.

\subsubsection{Statistical Analyses}

Unadjusted subgroup differences in time-invariant characteristics between medication adherence categories were tested with chi-square statistics. As medication adherence was measured every 120 days during pre- and postindex period, each individual had four observations. These four observations were not independent, therefore, we analyzed the relationship between adherence categories and CAD-related hospitalizations using multinomial logistic regression for repeated measures. As we are interested in describing changes in CAD-related hospitalization, given changes in adherence categories, while accounting for non-independence of observations within individuals, we selected the marginal models (i.e. population-averaged models) [182].

\subsubsection{Inverse Probability Treatment Weight (IPTW)}

As can be seen from Table 3.1, there were significant associations between medication adherence categories and for age, race/ethnicity, cancer type and sex, cancer stage, cancer treatment, concordant and discordant physical health conditions, mental health conditions, CAD severity, tobacco use, alcohol use, primary care visit, cardiologist visit, Part D Medicare coverage, and SEER region. Therefore, we derived IPTWs to balance the independent variables using a multinomial logistic regression on medication adherence categories during the period immediately after cancer diagnosis to take advantage of the availability of cancer-related variables (i.e. stage, cancer type, and cancer treatment). These IPTWs derived for each individual were then used as weights in CAD-related hospitalization analyses.

\subsubsection{Instrument Variable (IV) Regression}

It is plausible that there are some unobserved variables that can affect medication adherence and affect the relationship between medication adherence categories and CAD hospitalizations. IV regression is one approach to control for such unobserved selection bias. We selected the percentage of oncologists at the county-level as an 
instrument variable under the assumption that the effect of oncologists on CAD-related hospitalization occurs only through medication adherence categories. For this analysis, we dichotomized adherence categories into adherent to both medication and other; CAD hospitalization was dichotomized into CAD-related hospitalization and no CAD hospitalization that included no CAD hospitalization and other hospitalizations. 
Table 3.1. Multinomial Logistic Regression Models used to derive IPTW

\begin{tabular}{|c|c|c|c|c|c|c|c|c|c|c|c|c|}
\hline \multirow{2}{*}{ Characteristics } & \multicolumn{3}{|c|}{ No ADH 2 Rx } & \multicolumn{3}{|c|}{ ADH 1 of 2 Rx } & \multicolumn{3}{|c|}{ ADH 1 Rx } & \multicolumn{3}{|c|}{ No ADH 1 Rx } \\
\hline & AOR & $95 \% \mathrm{CI}$ & Sig & AOR & $95 \% \mathrm{CI}$ & Sig & AOR & $95 \% \mathrm{CI}$ & Sig & AOR & $95 \% \mathrm{CI}$ & Sig \\
\hline \multicolumn{13}{|l|}{ Age groups } \\
\hline $68-70$ years & 0.65 & $0.54,0.79$ & $* * *$ & 0.99 & $0.85,1.16$ & & 0.71 & $0.60,0.84$ & $* * *$ & 0.75 & $0.63,0.90$ & $* *$ \\
\hline 71-74 years & 0.79 & $0.67,0.93$ & $* *$ & 1.01 & $0.88,1.15$ & & 0.74 & $0.64,0.86$ & $* * *$ & 0.70 & $0.59,0.82$ & $* * *$ \\
\hline 75-79 years & 0.77 & $0.66,0.90$ & $* *$ & 1.01 & $0.89,1.16$ & & 0.81 & $0.70,0.93$ & $* *$ & 0.72 & $0.61,0.84$ & $* * *$ \\
\hline 80 years and older & Ref & & & & & & & & & & & \\
\hline \multicolumn{13}{|l|}{ Race/Ethnicity } \\
\hline African Americans & 1.88 & $1.52,2.32$ & $* * *$ & 1.17 & $0.96,1.43$ & & 1.42 & $1.16,1.74$ & $* * *$ & 1.90 & $1.54,2.35$ & $* * *$ \\
\hline Others & 1.47 & $1.20,1.80$ & $* * *$ & 1.17 & $0.98,1.39$ & & 1.07 & $0.88,1.30$ & & 1.12 & $0.90,1.38$ & \\
\hline Whites & Ref & & & & & & & & & & & \\
\hline \multicolumn{13}{|l|}{ Part D Medicare coverage } \\
\hline Exceeded & 2.93 & $1.96,4.38$ & $* * *$ & 1.51 & $1.14,1.99$ & $* *$ & 2.14 & $1.52,3.01$ & $* * *$ & 3.61 & $2.33,5.60$ & $* * *$ \\
\hline Not reached & 1.10 & $0.66,1.83$ & & 0.92 & $0.65,1.32$ & & 1.09 & $0.71,1.68$ & & 1.13 & $0.65,1.96$ & \\
\hline Reached & & & & & & & & & & & & \\
\hline \multicolumn{13}{|l|}{ Cancer diagnosis categories } \\
\hline Women with breast cancer & 0.86 & $0.71,1.04$ & & 1.08 & $0.92,1.25$ & & 1.54 & $1.31,1.81$ & $* * *$ & 1.33 & $1.11,1.60$ & $* *$ \\
\hline Women with colorectal cancer & 2.28 & $1.84,2.82$ & $* * *$ & 1.71 & $1.42,2.07$ & $* * *$ & 1.55 & $1.26,1.92$ & $* * *$ & 2.76 & $2.22,3.43$ & $* * *$ \\
\hline Men with colorectal cancer & 2.31 & $1.88,2.84$ & $* * *$ & 1.29 & $1.07,1.56$ & $* *$ & 1.11 & $0.89,1.39$ & & 2.37 & $1.90,2.95$ & $* * *$ \\
\hline Men with prostate cancer & Ref & & & & & & & & & & & \\
\hline \multicolumn{13}{|l|}{ Cancer stage } \\
\hline Stage $0 / \mathrm{I}$ & 0.61 & $0.50,0.74$ & $* * *$ & 0.89 & $0.75,1.05$ & & 0.80 & $0.67,0.97$ & $*$ & 0.67 & $0.55,0.82$ & $* * *$ \\
\hline Stage II & 0.76 & $0.64,0.90$ & $* *$ & 0.91 & $0.78,1.07$ & & 0.87 & $0.74,1.04$ & & 0.91 & $0.76,1.09$ & \\
\hline Stage III/IV & Ref & & & & & & & & & & & \\
\hline \multicolumn{13}{|l|}{ Cancer treatment } \\
\hline Cardiotoxic cancer treatment & 1.00 & $0.83,1.21$ & & 0.97 & $0.83,1.13$ & & 0.89 & $0.75,1.05$ & & 0.98 & $0.82,1.19$ & \\
\hline No cancer treatment & 1.17 & $0.98,1.39$ & & 0.92 & $0.80,1.07$ & & 0.98 & $0.84,1.14$ & & 1.01 & $0.84,1.20$ & \\
\hline Non-cardiotoxic cancer treatment & Ref & & & 0.91 & $0.83,1.00$ & * & 0.89 & $0.81,0.97$ & * & 0.99 & $0.88,1.10$ & \\
\hline \multicolumn{13}{|l|}{ Discordant PHC } \\
\hline Yes & 1.29 & $1.14,1.47$ & $* * *$ & 1.15 & $1.04,1.28$ & $* *$ & 1.34 & $1.20,1.50$ & $* * *$ & 1.57 & $1.39,1.79$ & $* * *$ \\
\hline No & Ref & & & & & & & & & & & \\
\hline \multicolumn{13}{|l|}{ Concordant PHC } \\
\hline Yes & 0.66 & $0.49,0.90$ & $* *$ & 0.84 & $0.65,1.10$ & & 0.49 & $0.38,0.63$ & $* * *$ & 0.32 & $0.25,0.41$ & $* * *$ \\
\hline No & Ref & & & & & & & & & & & \\
\hline \multicolumn{13}{|l|}{ МHC } \\
\hline Yes & 1.60 & $1.36,1.89$ & $* * *$ & 1.16 & $0.99,1.34$ & & 1.20 & $1.02,1.41$ & $*$ & 1.77 & $1.50,2.09$ & $* * *$ \\
\hline No & Ref & & & & & & & & & & & \\
\hline Tobacco use & & & & & & & & & & & & \\
\hline Yes & 1.39 & $1.04,1.85$ & $*$ & 1.16 & $0.90,1.51$ & & 0.98 & $0.73,1.31$ & & 1.12 & $0.83,1.52$ & \\
\hline
\end{tabular}




\begin{tabular}{|c|c|c|c|c|c|c|c|c|c|c|c|c|}
\hline \multirow{2}{*}{ Characteristics } & \multicolumn{3}{|c|}{ No ADH 2 Rx } & \multicolumn{3}{|c|}{ ADH 1 of 2 Rx } & \multicolumn{3}{|c|}{ ADH 1 Rx } & \multicolumn{3}{|c|}{ No ADH 1 Rx } \\
\hline & AOR & $95 \% \mathrm{CI}$ & Sig & AOR & $95 \%$ CI & Sig & AOR & $95 \% \mathrm{CI}$ & Sig & AOR & $95 \%$ CI & Sig \\
\hline No & Ref & & & & & & & & & & & \\
\hline \multicolumn{13}{|l|}{ Alcohol use } \\
\hline Yes & 1.22 & $0.67,2.20$ & & 0.99 & $0.58,1.71$ & & 1.35 & $0.78,2.34$ & & 2.53 & $1.53,4.19$ & $* * *$ \\
\hline No & Ref & & & & & & & & & & & \\
\hline \multicolumn{13}{|l|}{ Primary care visit } \\
\hline Yes & 1.34 & $1.11,1.61$ & $* *$ & 1.14 & $0.99,1.32$ & & 1.00 & $0.87,1.17$ & & 1.05 & $0.88,1.24$ & \\
\hline No & Ref & & & & & & & & & & & \\
\hline \multicolumn{13}{|l|}{ Cardiologist visit } \\
\hline Yes & 1.03 & $0.90,1.18$ & & 1.08 & $0.97,1.21$ & & 0.65 & $0.58,0.73$ & $* * *$ & 0.73 & $0.64,0.83$ & $* * *$ \\
\hline No & Ref & & & & & & & & & & & \\
\hline CAD severity & 1.15 & $0.85,1.56$ & & 0.98 & $0.75,1.27$ & & 0.61 & $0.46,0.83$ & $* *$ & 0.69 & $0.50,0.96$ & $*$ \\
\hline \multicolumn{13}{|l|}{ SEER Region } \\
\hline Northeast & 1.04 & $0.88,1.22$ & & 1.07 & $0.94,1.22$ & & 1.15 & $1.00,1.33$ & $*$ & 0.92 & $0.78,1.08$ & \\
\hline South & 1.24 & $1.06,1.46$ & $* *$ & 1.17 & $1.02,1.33$ & $*$ & 1.05 & $0.91,1.22$ & & 1.10 & $0.94,1.29$ & \\
\hline North-central & 0.92 & $0.76,1.11$ & & 1.01 & $0.87,1.18$ & & 0.96 & $0.82,1.14$ & & 0.76 & $0.62,0.92$ & $* *$ \\
\hline West & Ref & & & & & & & & & & & \\
\hline
\end{tabular}

Based on 12,096 elderly fee-for-service Medicare beneficiaries with pre-existing CAD and incident breast, colorectal, and prostate cancer. Weights were derived using the Inverse Probability Treatment Weighting approach.

\section{Description of medication use and adherence}

No ADH 2 Rx: Not adherent to both statins and ACEIs/ARBs/beta-blockers;

ADH 1 of 2 Rx: Adherent to either statins or ACEIs/ARBs/beta-blockers;

ADH 1 Rx: Use of one medication class and adherent to that class;

No ADH 1 Rx: Use of one medication class and not adherent to that medication class

Asterisks represent significant differences in study population characteristics (time-invariant). Reference category is Adherent to both statins and

\section{ACEIs/ARBs/beta-blockers}

${ }^{* * *} \mathrm{p}<0.001 ; * * 0.001 \leq \mathrm{p}<0.01 ; * 0.01 \leq \mathrm{p}<0.05$ 
Time-invariant characteristics include cancer diagnosis categories, age, race/ethnicity, concordant and discordant physical health conditions, CAD severity, SMI, SEER region. Time-variant characteristics include tobacco use, alcohol use, depression, anxiety, and routine follow-up with primary care physician and/or cardiologist, and Part D Medicare coverage.

Concordant physical health conditions consisted of diabetes, hyperlipidemia, hypertension, stroke, cardiac arrhythmia, and congestive heart failure. Discordant physical health conditions consisted of arthritis, asthma, chronic obstructive pulmonary disease, osteoporosis, dementia, human immunodeficiency virus, and hepatitis. Mental health conditions include anxiety, depression, SMI (schizophrenia, bipolar disorder, and psychoses)

\section{Abbreviations}

ACEIs: Angiotensin-converting enzyme inhibitors; ARBs: Angiotensin II receptor blockers; CAD: Coronary artery disease; FFS: Fee-for-service; IPTW: inverse probability treatment weighting; MHC: Mental health conditions; PHC: Physical health conditions; Ref: Reference; Rx: Prescription; SEER: Surveillance,

Epidemiology and End Results 


\subsection{Results}

\subsubsection{Characteristics of the Study Population}

The study population comprised 12,096 elderly FFS Medicare beneficiaries with pre-existing CAD and incident cancer diagnosis. There were 33.1\% women with breast cancer, $14.1 \%$ women with colorectal cancer; $11.6 \%$ men with colorectal cancer, and 41.2\% men with prostate cancer (Table 3.2). Nearly one-third (29.5\%) were diagnosed with stage 0 or I. Only $16.8 \%$ received no cancer treatment.

3.4.1.1 Characteristics of the Study Population by Medication Adherence Categories - Before and After IPTW Adjustment

Table 3.3 summarizes the characteristics of the cohort by adherence to statins and/or ACEIs/ARBs/betablockers before and after IPTW adjustment. Prior to IPTW adjustment, there were statistically significant differences in age, race/ethnicity, cancer type and sex, cancer stage, cancer treatment, concordant physical conditions, discordant physical conditions, mental health conditions, tobacco use, alcohol use, any primary care visit, cardiologist visit, Part D Medicare coverage, and SEER region between the medication adherence groups. After IPTW adjustment, there were no significant differences in these variables between the medication adherence groups.

Adherence to both statins and ACEIs/ARBs/beta-blockers was estimated at 31.2\% during the 120-day period immediately after cancer diagnosis; $13.7 \%$ were not adherent to both medication classes during the same period.

Table 3.4 summarizes the CAD-related hospitalizations over time. The prevalence of CAD-related hospitalizations was $27.4 \%$ during the 120 -day period immediately after cancer diagnosis and this percentage declined to $10.6 \%$ during the last four months of post-cancer period. 
Table 3.2. Selected Characteristics of Study Population

\begin{tabular}{|c|c|c|}
\hline \multirow{2}{*}{ Characteristics } & \multicolumn{2}{|c|}{ Total population } \\
\hline & $\mathbf{N}$ & $\%$ \\
\hline \multicolumn{3}{|l|}{ Age groups } \\
\hline $68-70$ years & 1,983 & 16.4 \\
\hline $71-74$ years & 2,887 & 23.9 \\
\hline 75-79 years & 3,158 & 26.1 \\
\hline 80 years and older & 4,068 & 33.6 \\
\hline \multicolumn{3}{|l|}{ Race/Ethnicity } \\
\hline Whites & 9,928 & 82.1 \\
\hline African Americans & 1,013 & 8.4 \\
\hline Others & 1,155 & 9.5 \\
\hline \multicolumn{3}{|l|}{ Cancer diagnosis } \\
\hline Women with breast cancer & 4,007 & 33.1 \\
\hline Women with colorectal cancer & 1,705 & 14.1 \\
\hline Men with colorectal cancer & 1,399 & 11.6 \\
\hline Men with prostate cancer & 4,985 & 41.2 \\
\hline \multicolumn{3}{|l|}{ Cancer stage } \\
\hline Stage $0 / \mathrm{I}$ & 3,565 & 29.5 \\
\hline Stage II & 6,572 & 54.3 \\
\hline Stage III/IV & 1,959 & 16.2 \\
\hline \multicolumn{3}{|l|}{ Cancer treatment } \\
\hline Cardiotoxic cancer treatment & 4,821 & 34.9 \\
\hline Non-cardiotoxic cancer treatment & 6,668 & 48.3 \\
\hline No cancer treatment & 2,322 & 16.8 \\
\hline \multicolumn{3}{|l|}{ SEER Region } \\
\hline Northeast & 2,749 & 22.7 \\
\hline South & 2,977 & 24.6 \\
\hline North-central & 1,691 & 14.0 \\
\hline West & 4,679 & 38.7 \\
\hline \multicolumn{3}{|l|}{ Index year } \\
\hline 2008 & 2,966 & 24.5 \\
\hline 2009 & 2,975 & 24.6 \\
\hline 2010 & 2,992 & 24.7 \\
\hline 2011 & 3,163 & 26.1 \\
\hline
\end{tabular}

Note: Based on 12,096 elderly fee-for-service Medicare beneficiaries with pre-existing CAD and incident breast, colorectal, and prostate cancer between 2008 and 2011. Table includes time-invariant characteristics.

Abbreviation: SEER: Surveillance, Epidemiology and End Results 
Table 3.3. Unweighted and Weighted Percent of Selected Characteristics by Adherence to Statins and/or ACEIs/ARBs/Beta-blockers

\begin{tabular}{|c|c|c|c|c|c|c|c|c|c|c|c|c|}
\hline \multirow[b]{2}{*}{ Characteristics } & \multicolumn{6}{|c|}{ Before IPTW (\%) } & \multicolumn{6}{|c|}{ After IPTW (Wt. \%) } \\
\hline & $\begin{array}{c}\text { ADH } 2 \\
\text { Rx }\end{array}$ & $\begin{array}{c}\text { No } \\
\text { ADH } 2 \\
\text { Rx } \\
\end{array}$ & $\begin{array}{l}\text { ADH } 1 \\
\text { of } 2 \text { Rx }\end{array}$ & $\begin{array}{c}\text { ADH } 1 \\
\text { Rx }\end{array}$ & $\begin{array}{c}\text { No } \\
\text { ADH 1 } \\
\text { Rx } \\
\end{array}$ & Sig & $\begin{array}{c}\text { ADH } 2 \\
\text { Rx }\end{array}$ & $\begin{array}{c}\text { No } \\
\text { ADH } 2 \\
\text { Rx } \\
\end{array}$ & $\begin{array}{l}\text { ADH } 1 \\
\text { of } 2 \text { Rx }\end{array}$ & $\begin{array}{c}\text { ADH } 1 \\
\text { Rx }\end{array}$ & $\begin{array}{c}\text { No } \\
\text { ADH } 1 \\
\text { Rx } \\
\end{array}$ & Sig \\
\hline Age groups & & & & & & $* * *$ & & & & & & NS \\
\hline $68-70$ years & 18.0 & 13.7 & 17.1 & 14.9 & 16.3 & & 16.5 & 16.8 & 16.3 & 16.5 & 16.6 & \\
\hline $71-74$ years & 25.7 & 23.3 & 24.9 & 22.0 & 21.1 & & 24.0 & 23.8 & 23.9 & 24.0 & 24.0 & \\
\hline $75-79$ years & 27.3 & 24.6 & 27.2 & 25.9 & 23.4 & & 26.4 & 26.0 & 25.9 & 25.8 & 26.3 & \\
\hline 80 years and older & 29.0 & 38.4 & 30.8 & 37.2 & 39.2 & & 33.1 & 33.4 & 33.8 & 33.8 & 33.0 & \\
\hline Race/Ethnicity & & & & & & $* * *$ & & & & & & NS \\
\hline Whites & 84.5 & 77.6 & 82.7 & 82.4 & 79.6 & & 82.2 & 81.6 & 82.2 & 81.7 & 82.3 & \\
\hline African Americans & 6.4 & 10.8 & 7.6 & 8.8 & 11.2 & & 8.4 & 8.4 & 8.2 & 8.5 & 8.5 & \\
\hline Others & 9.1 & 11.6 & 9.8 & 8.8 & 9.3 & & 9.4 & 10.0 & 9.5 & 9.7 & 9.2 & \\
\hline Part D Medicare coverage & & & & & & $* * *$ & & & & & & NS \\
\hline Exceeded & 5.6 & 2.7 & 3.9 & 3.4 & 2.4 & & 4.1 & 4.3 & 4.0 & 4.1 & 3.8 & \\
\hline Not reached & 90.5 & 95.4 & 93.1 & 94.5 & 96.1 & & 93.2 & 93.1 & 93.3 & 93.1 & 93.4 & \\
\hline Reached & 3.8 & 1.9 & 3.0 & 2.1 & 1.5 & & 2.8 & 2.6 & 2.7 & 2.8 & 2.7 & \\
\hline Cancer diagnosis categories & & & & & & $* * *$ & & & & & & NS \\
\hline Women with breast cancer & 33.8 & 23.3 & 32.5 & 41.6 & 31.2 & & 34.0 & 33.5 & 33.2 & 33.5 & 33.5 & \\
\hline Women with colorectal cancer & 9.3 & 21.2 & 14.8 & 12.1 & 19.4 & & 13.7 & 13.9 & 14.1 & 13.8 & 13.7 & \\
\hline Men with colorectal cancer & 9.5 & 19.0 & 11.0 & 7.9 & 14.6 & & 11.3 & 11.4 & 11.4 & 11.7 & 11.4 & \\
\hline Men with prostate cancer & 47.4 & 36.5 & 41.7 & 38.4 & 34.8 & & 41.0 & 41.3 & 41.3 & 41.0 & 41.3 & \\
\hline Cancer stage & & & & & & $* * *$ & & & & & & NS \\
\hline Stage 0/I & 29.2 & 25.9 & 30.3 & 32.6 & 27.9 & & 29.8 & 29.4 & 29.4 & 29.4 & 29.8 & \\
\hline Stage II & 57.4 & 51.9 & 53.8 & 52.5 & 53.0 & & 54.0 & 55.0 & 54.4 & 54.3 & 54.1 & \\
\hline Stage III/IV & 13.4 & 22.1 & 15.8 & 14.9 & 19.1 & & 16.2 & 15.6 & 16.2 & 16.3 & 16.1 & \\
\hline Cancer treatment & & & & & & $* * *$ & & & & & & NS \\
\hline Cardiotoxic cancer treatment & 37.3 & 30.3 & 36.5 & 35.4 & 33.2 & & 35.7 & 36.1 & 35.4 & 35.3 & 35.5 & \\
\hline Non-cardiotoxic cancer treatment & 16.8 & 14.7 & 16.7 & 16.7 & 16.0 & & 16.5 & 16.8 & 16.3 & 16.5 & 16.2 & \\
\hline No cancer treatment & 45.9 & 55.0 & 46.8 & 47.8 & 50.9 & & 47.9 & 47.1 & 48.3 & 48.2 & 48.3 & \\
\hline Discordant PHC & & & & & & $* * *$ & & & & & & NS \\
\hline Yes & 35.7 & 43.4 & 39.6 & 43.2 & 47.1 & & 40.7 & 41.0 & 40.9 & 40.9 & 40.8 & \\
\hline No & 64.3 & 56.6 & 60.4 & 56.8 & 52.9 & & 59.3 & 59.0 & 59.1 & 59.1 & 59.2 & \\
\hline Concordant PHC & & & & & & $* * *$ & & & & & & NS \\
\hline Yes & 96.6 & 95.8 & 96.3 & 93.8 & 91.5 & & 95.2 & 95.4 & 95.2 & 95.3 & 95.1 & \\
\hline No & 3.4 & 4.2 & 3.7 & 6.2 & 8.5 & & 4.8 & 4.6 & 4.8 & 4.7 & 4.9 & \\
\hline MHC & & & & & & $*$ & & & & & & NS \\
\hline Yes & 11.9 & 18.8 & 14.1 & 14.6 & 20.5 & & 15.0 & 14.3 & 14.9 & 15.0 & 14.8 & \\
\hline No & 88.1 & 81.2 & 85.9 & 85.4 & 79.5 & & 85.0 & 85.7 & 85.1 & 85.0 & 85.2 & \\
\hline
\end{tabular}




\begin{tabular}{|c|c|c|c|c|c|c|c|c|c|c|c|c|}
\hline \multirow[b]{2}{*}{ Characteristics } & \multicolumn{6}{|c|}{ Before IPTW (\%) } & \multicolumn{6}{|c|}{ After IPTW (Wt. \%) } \\
\hline & $\begin{array}{c}\text { ADH } 2 \\
\text { Rx }\end{array}$ & $\begin{array}{c}\text { No } \\
\text { ADH } 2 \\
\text { Rx }\end{array}$ & $\begin{array}{l}\text { ADH } 1 \\
\text { of } 2 \text { Rx }\end{array}$ & $\begin{array}{c}\text { ADH } 1 \\
\text { Rx }\end{array}$ & $\begin{array}{c}\text { No } \\
\text { ADH } 1 \\
\text { Rx }\end{array}$ & Sig & $\begin{array}{c}\text { ADH } 2 \\
\text { Rx }\end{array}$ & $\begin{array}{c}\text { No } \\
\text { ADH } 2 \\
\text { Rx } \\
\end{array}$ & $\begin{array}{l}\text { ADH } 1 \\
\text { of } 2 \text { Rx }\end{array}$ & $\begin{array}{c}\text { ADH } 1 \\
\text { Rx }\end{array}$ & $\begin{array}{c}\text { No } \\
\text { ADH } 1 \\
\text { Rx } \\
\end{array}$ & Sig \\
\hline Tobacco use & & & & & & $* *$ & & & & & & NS \\
\hline Yes & 3.4 & 5.3 & 4.3 & 3.5 & 4.7 & & 3.9 & 4.4 & 4.0 & 4.0 & 3.7 & \\
\hline No & 96.6 & 94.7 & 95.7 & 96.5 & 95.3 & & 96.1 & 95.6 & 96.0 & 96.0 & 96.3 & \\
\hline Alcohol use & & & & & & $* * *$ & & & & & & NS \\
\hline Yes & 0.8 & 1.1 & 0.9 & 1.0 & 2.2 & & 0.9 & 1.0 & 1.1 & 1.0 & 0.9 & \\
\hline No & 99.2 & 98.9 & 99.1 & 99.0 & 97.8 & & 99.1 & 99.0 & 98.9 & 99.0 & 99.1 & \\
\hline Primary care visit & & & & & & $* * *$ & & & & & & NS \\
\hline Yes & 83.0 & 89.4 & 85.9 & 84.7 & 86.8 & & 85.2 & 84.7 & 85.3 & 85.4 & 85.3 & \\
\hline No & 17.0 & 10.6 & 14.1 & 15.3 & 13.2 & & 14.8 & 15.3 & 14.7 & 14.6 & 14.7 & \\
\hline Cardiologist visit & & & & & & $* * *$ & & & & & & NS \\
\hline Yes & 68.1 & 73.2 & 71.1 & 59.6 & 64.5 & & 66.8 & 67.6 & 67.2 & 67.4 & 66.7 & \\
\hline No & 31.9 & 26.8 & 28.9 & 40.4 & 35.5 & & 33.2 & 32.4 & 32.8 & 32.6 & 33.3 & \\
\hline SEER Region & & & & & & $*$ & & & & & & NS \\
\hline Northeast & 22.9 & 21.4 & 22.8 & 24.6 & 20.5 & & 22.5 & 21.9 & 22.7 & 22.4 & 22.7 & \\
\hline South & 23.4 & 25.8 & 25.5 & 23.4 & 26.6 & & 25.0 & 25.6 & 24.7 & 24.9 & 24.7 & \\
\hline North-central & 14.8 & 13.1 & 13.8 & 14.4 & 12.3 & & 13.9 & 13.6 & 13.9 & 13.8 & 14.3 & \\
\hline West & 38.8 & 39.7 & 37.9 & 37.6 & 40.5 & & 38.6 & 38.9 & 38.7 & 39.0 & 38.4 & \\
\hline
\end{tabular}

Note: Based on 12,096 elderly fee-for-service Medicare beneficiaries with pre-existing CAD and incident breast, colorectal, and prostate cancer diagnosis.

Weights were derived using the Inverse Probability Treatment Weighting approach.

Description of medication use and adherence

ADH 2 Rx: Adherent to both statins and ACEIs/ARBs/beta-blockers;

No ADH 2 Rx: Not adherent to both statins and ACEIs/ARBs/beta-blockers;

ADH 1 of 2 Rx: Adherent to either statins or ACEIs/ARBs/beta-blockers;

ADH 1 Rx: Use of one medication class and adherent to that class;

No ADH 1 Rx: Use of one medication class and not adherent to that medication class

Asterisks represent significant differences in time-invariant patient-level characteristics based on chi-square tests

${ }^{* * *} \mathrm{p}<0.001 ; * * 0.001 \leq \mathrm{p}<0.01 ; * 0.01 \leq \mathrm{p}<0.05$. Column percentages are reported. 
Time-invariant characteristics include cancer diagnosis categories, age, race/ethnicity, concordant and discordant physical health conditions, SMI, SEER region. Time-variant characteristics include tobacco use, alcohol use, depression, anxiety, and routine follow-up with primary care physician and/or cardiologist, and Part D Medicare coverage.

Concordant physical health conditions consisted of diabetes, hyperlipidemia, hypertension, stroke, cardiac arrhythmia, and congestive heart failure. Discordant physical health conditions consisted of arthritis, asthma, chronic obstructive pulmonary disease, osteoporosis, dementia, human immunodeficiency virus, and hepatitis. Mental health conditions include anxiety, depression, SMI (schizophrenia, bipolar disorder, and psychoses)

\section{Abbreviations}

ACEIs: Angiotensin-converting enzyme inhibitors; ARBs: Angiotensin II receptor blockers; CAD: Coronary artery disease; FFS: Fee-for-service; IPTW: inverse probability treatment weighting; MHC: Mental health conditions; PHC: Physical health conditions; Rx: Prescription; SEER: Surveillance, Epidemiology and End Results; Wt.: Weighted 
Table 3.4. Weighted percentage with CAD-related hospitalizations over different time periods

\begin{tabular}{|c|c|c|c|c|}
\hline \multirow{3}{*}{$\begin{array}{l}\text { Use and adherence to CAD-related } \\
\text { pharmacotherapy }\end{array}$} & $\begin{array}{c}\text { Pre-cancer dx } \\
\text { period }\end{array}$ & $\begin{array}{c}\text { Initial cancer } \\
\text { tx period }\end{array}$ & \multicolumn{2}{|c|}{ Post-tx period } \\
\hline & $\mathbf{t}_{\mathbf{0}}$ & $\mathbf{t}_{1}$ & $\mathbf{t}_{2}$ & $\mathbf{t}_{3}$ \\
\hline & Wt. \% & Wt. \% & Wt. \% & Wt. \% \\
\hline \multicolumn{5}{|c|}{ CAD-related hospitalization } \\
\hline ADH 2 Rx & 7.5 & 28.6 & 8.9 & 7.6 \\
\hline No ADH 2 Rx & 10.4 & 31.4 & 18.0 & 14.7 \\
\hline ADH 1 of 2 Rx & 8.5 & 28.1 & 12.6 & 11.2 \\
\hline ADH 1 Rx & 5.7 & 19.9 & 7.3 & 7.1 \\
\hline No ADH 1 Rx & 7.6 & 22.7 & 9.7 & 10.2 \\
\hline \multicolumn{5}{|c|}{ Other hospitalization } \\
\hline ADH 2 Rx & 2.1 & 14.2 & 3.5 & 2.6 \\
\hline No ADH 2 Rx & 3.6 & 18.3 & 7.0 & 6.2 \\
\hline ADH 1 of 2 Rx & 2.8 & 17.4 & 3.9 & 4.4 \\
\hline ADH 1 Rx & 3.2 & 22.7 & 5.7 & 4.0 \\
\hline No ADH 1 Rx & 4.3 & 23.8 & 8.0 & 6.3 \\
\hline
\end{tabular}

Note: Based on 12,096 elderly fee-for-service Medicare beneficiaries with pre-existing CAD and incident breast, colorectal, and prostate cancer diagnosis. Weights were derived using the Inverse Probability Treatment Weighting approach.

Description of medication use and adherence

ADH 2 Rx: Adherent to both statins and ACEIs/ARBs/beta-blockers;

No ADH 2 Rx: Not adherent to both statins and ACEIs/ARBs/beta-blockers;

ADH 1 of 2 Rx: Adherent to either statins or ACEIs/ARBs/beta-blockers;

ADH 1 Rx: Use of one medication class and adherent to that class;

No ADH 1 Rx: Use of one medication class and not adherent to that medication class

\section{$\underline{\text { Abbreviations }}$}

CAD: Coronary artery disease 


\subsubsection{Unadjusted and Adjusted Associations between Adherence to Statins and/or ACEIs/ARBs/Beta- blockers and CAD-related Hospitalization}

Table 3.5 displays the results from the unadjusted and adjusted multinomial logistic regression models with IPTW for repeated measures on CAD-related hospitalization. The adjusted model controlled for time, age, race/ethnicity, census tract education level and poverty status, marital status, Part D Medicare coverage, concordant and discordant physical health conditions, and mental health conditions, cancer type and sex, cancer stage, cancer treatment, tobacco use, alcohol use, visits to primary care/cardiologist, SEER region, and county metro status. Reference category for the dependent variable was no $C A D$ hospitalization.

In unadjusted analyses, those not adherent to both medication classes [OR $=1.67 ; 95 \% \mathrm{CI}=1.58,1.76 ; P<$ $0.0001]$ and those who were adherent to one (out of two) medication class [OR $=1.22 ; 95 \% \mathrm{CI}=1.15,1.30 ; P<$ 0.0001] were more likely to have CAD-related hospitalization, compared to those who were adherent to both medication classes. However, those with the use of one medication class and adherent to that medication class were significantly less likely to have CAD-related hospitalization compared to those who were adherent to both medication classes [OR $=0.76 ; 95 \% \mathrm{CI}=0.66,0.86 ; P<0.0001]$.

Consistent with the findings from unadjusted model, compared to those who were adherent to both medication classes, those not adherent to both medication classes [AOR $=1.82 ; 95 \% \mathrm{CI}=1.72,1.92 ; \mathrm{P}<0.0001]$ and those who were adherent to one (out of two) medication class [AOR $=1.26$; 95\% $\mathrm{CI}=1.17,1.34 ; \mathrm{P}<0.0001$ ] were more likely to have CAD-related hospitalization in the adjusted model. Further, those with the use of one medication class and adherent to that medication class were significantly less likely to have CAD-related hospitalization compared to those who were adherent to both medication classes [AOR $=0.80 ; 95 \% \mathrm{CI}=0.70,0.90 ; \mathrm{P}<0.0001]$. However, those with use of one medication class and not adherent to that medication class [AOR $=1.20 ; 95 \% \mathrm{CI}=1.09,1.31$; $P=0.005]$ were more likely to have CAD-related hospitalization. 
Table 3.5. Odds Ratios, Adjusted Odds Ratios, and 95\% Confidence Intervals of Medication Adherence Categories from Multinomial Logistic Regression Models with Repeated Measures on Hospitalization Categories - IPTW (Reference Group = No Hospitalizations)

\begin{tabular}{|c|c|c|c|c|c|c|}
\hline \multirow{2}{*}{ Variables } & \multicolumn{3}{|c|}{ CAD-related hospitalization } & \multicolumn{3}{|c|}{ Other hospitalization } \\
\hline & OR & $95 \% \mathrm{CI}$ & Sig & OR & $95 \% \mathrm{CI}$ & Sig \\
\hline \multicolumn{7}{|c|}{ Unadjusted analysis } \\
\hline \multicolumn{7}{|l|}{ Medication adherence categories } \\
\hline No ADH 2 Rx & 1.67 & {$[1.58,1.76]$} & $* * *$ & 1.88 & {$[1.75,2.00]$} & *** \\
\hline ADH 1 of $2 \mathrm{Rx}$ & 1.22 & {$[1.15,1.30]$} & $* * *$ & 1.36 & {$[1.25,1.46]$} & *** \\
\hline ADH 1 Rx & 0.76 & {$[0.66,0.86]$} & $* * *$ & 1.59 & {$[1.48,1.70]$} & *** \\
\hline No ADH $1 \mathrm{Rx}$ & 1.05 & {$[0.94,1.15]$} & & 2.10 & {$[1.99,2.22]$} & $* * *$ \\
\hline ADH 2 Rx & & Reference & & & Reference & \\
\hline \multicolumn{7}{|l|}{ Cancer diagnosis categories } \\
\hline Women with breast cancer & 1.15 & {$[1.07,1.23]$} & $* * *$ & 1.61 & {$[1.52,1.70]$} & *** \\
\hline Women with colorectal cancer & 3.39 & {$[3.31,3.47]$} & $* * *$ & 4.23 & {$[4.14,4.32]$} & $* * *$ \\
\hline Men with colorectal cancer & 3.60 & {$[3.52,3.68]$} & $* * *$ & 2.91 & {$[2.80,3.02]$} & *** \\
\hline Men with prostate cancer & & Reference & & & Reference & \\
\hline \multicolumn{7}{|l|}{ Cancer stage } \\
\hline Stage $0 / \mathrm{I}$ & 0.93 & {$[0.87,0.99]$} & * & 0.82 & {$[0.77,0.88]$} & *** \\
\hline Stage III/IV & 2.04 & {$[1.97,2.12]$} & $* * *$ & 2.31 & {$[2.22,2.40]$} & *** \\
\hline Stage II & & Reference & & & Reference & \\
\hline \multicolumn{7}{|l|}{ Cancer treatment } \\
\hline Cardiotoxic cancer treatment & 1.30 & {$[1.16,1.44]$} & $* * *$ & 2.36 & {$[2.16,2.56]$} & $* * *$ \\
\hline No cancer treatment & 0.88 & {$[0.76,1.00]$} & $*$ & 1.11 & {$[0.93,1.29]$} & \\
\hline Non-cardiotoxic cancer & & Reference & & & Reference & \\
\hline \multicolumn{7}{|c|}{ Adjusted analysis } \\
\hline Variables & AOR & $95 \% \mathrm{CI}$ & Sig & AOR & $95 \% \mathrm{CI}$ & Sig \\
\hline \multicolumn{7}{|l|}{ Medication adherence categories } \\
\hline No ADH 2 Rx & 1.82 & {$[1.72,1.92]$} & $* * *$ & 2.00 & {$[1.87,2.13]$} & $* * *$ \\
\hline $\mathrm{ADH} 1$ of $2 \mathrm{Rx}$ & 1.26 & {$[1.17,1.34]$} & $* * *$ & 1.39 & {$[1.28,1.49]$} & $* * *$ \\
\hline ADH 1 Rx & 0.80 & {$[0.70,0.90]$} & $* * *$ & 1.65 & {$[1.54,1.76]$} & $* * *$ \\
\hline No ADH $1 \mathrm{Rx}$ & 1.20 & {$[1.09,1.31]$} & $* *$ & 2.34 & {$[2.22,2.46]$} & $* * *$ \\
\hline ADH 2 Rx & & Reference & & & Reference & \\
\hline \multicolumn{7}{|l|}{ Cancer diagnosis categories } \\
\hline Women with breast cancer & 1.18 & {$[1.08,1.29]$} & $* *$ & 1.59 & {$[1.47,1.71]$} & $* * *$ \\
\hline Women with colorectal cancer & 3.31 & {$[3.20,3.42]$} & $* * *$ & 4.20 & {$[4.07,4.33]$} & $* * *$ \\
\hline Men with colorectal cancer & 3.78 & {$[3.67,3.88]$} & $* * *$ & 2.80 & {$[2.67,2.93]$} & $* * *$ \\
\hline Men with prostate cancer & & Reference & & & Reference & \\
\hline \multicolumn{7}{|l|}{ Cancer stage } \\
\hline Stage $0 / \mathrm{I}$ & 0.65 & {$[0.55,0.74]$} & $* * *$ & 0.71 & {$[0.60,0.81]$} & *** \\
\hline Stage III/IV & 1.25 & {$[1.15,1.34]$} & $* * *$ & 1.37 & {$[1.26,1.47]$} & $* * *$ \\
\hline Stage II & & Reference & & & Reference & \\
\hline \multicolumn{7}{|l|}{ Cancer treatment } \\
\hline Cardiotoxic cancer treatment & 1.31 & {$[1.14,1.49]$} & $* *$ & 2.14 & {$[1.91,2.37]$} & *** \\
\hline No cancer treatment & 0.84 & {$[0.69,0.98]$} & $*$ & 1.07 & {$[0.86,1.28]$} & \\
\hline $\begin{array}{l}\text { Non-cardiotoxic cancer } \\
\text { treatment }\end{array}$ & & Reference & & & Reference & \\
\hline
\end{tabular}

Note: Based on 12,096 elderly fee-for-service Medicare beneficiaries with pre-existing CAD and incident breast, colorectal, and prostate cancer diagnosis. Weights were derived using the Inverse Probability Treatment Weighting approach. 
Description of medication use and adherence

ADH 2 Rx: Adherent to both statins and ACEIs/ARBs/beta-blockers;

No ADH 2 Rx: Not adherent to both statins and ACEIs/ARBs/beta-blockers;

ADH 1 of 2 Rx: Adherent to either statins or ACEIs/ARBs/beta-blockers;

ADH 1 Rx: Use of one medication class and adherent to that class;

No ADH 1 Rx: Use of one medication class and not adherent to that medication class

Asterisks represent significant differences in medication adherence groups, derived from multinomial logistic regression models with repeated measures. Reference category for dependent variable is no hospitalization. $* * * \mathrm{p}<0.001 ; * * 0.001 \leq \mathrm{p}<0.01 ; * 0.01 \leq \mathrm{p}<0.05$.

ORs are reported for unadjusted GEE model and AORs are reported for adjusted GEE model.

Adjusted model controlled for independent variables. Time-invariant characteristics include cancer diagnosis categories, age, race/ethnicity, poverty status, high school education, concordant and discordant physical health conditions, SMI, SEER region, and county-level characteristics. Time-variant characteristics include, tobacco use, alcohol use, depression, anxiety, and routine follow-up with primary care physician and/or cardiologist, and Part D Medicare coverage.

\section{Abbreviations}

AOR: Adjusted odds ratio; CAD: Coronary artery disease; CI: Confidence interval; FFS: Fee-for-service; GEE: Generalized estimating equations; OR: Odds ratio; IPTW: Inverse Probability treatment weighting; Sig: Significance; SEER: Surveillance, Epidemiology, and End Result. 


\subsubsection{Sensitivity Analyses}

We found that the percent of oncologists at the county-level was a strong and valid instrument variable. The variable oncologists at the county-level were significantly associated with medication adherence [OR $=4.27 ; 95 \%$ $\mathrm{CI}=2.15,8.47 ; \mathrm{P}<0.0001$ ] and medication adherence was an endogenous variable [Wald test, $\mathrm{P}<0.0001$ ]. Results from IV regression revealed that Medicare beneficiaries in other medication adherence category (not adherent to one or both medication classes) were more likely to have CAD-related hospitalization compared to those who were adherent to both medication classes [Beta $=1.20 ; \mathrm{SE}=0.10 ; \mathrm{P}<0.0001$ ]. The results did not change when medication adherence was categorized into three categories, those not adherent to one or both medication classes were more likely to have CAD-related hospitalization [Beta $=1.22$; $\mathrm{SE}=0.12 ; \mathrm{P}<0.0001$ ], whereas those with single medication use and adherent to that medication [Beta $=-0.98$; $\mathrm{SE}=0.13 ; \mathrm{P}<0.0001$ ] were less likely to have CAD-related hospitalization when compared to those adherent to both medications.

Table 3.6 displays the AORs and 95\% CI for from the adjusted multinomial logistic regressions on CADrelated hospitalization that accounted for IPTW and repeated measures, where CAD-related hospitalizations were identified from primary diagnosis. There were some similarities and differences in comparison to GEE model with hospitalization identified from both primary and secondary diagnosis (primary analyses).

Consistent with the primary analyses (adjusted model), those who were not adherent to both medication classes [AOR $=2.57 ; 95 \% \mathrm{CI}=2.41,2.74 ; P<0.0001$ ], adherent to one (out of two) medication class [AOR = 1.42; 95\%

$\mathrm{CI}=1.27,1.57 ; P<0.0001$ ], or were using single medication class and not adherent to that medication class [AOR $=1.42 ; 95 \% \mathrm{CI}=1.27,1.57 ; P=0.0002]$ were more likely to have CAD-related hospitalization compared to those who were adherent to both medication classes. However, in contrast to the primary analyses, there was no significant difference in those using one medication class and adherent to that medication class compared to those who were adherent to both medication classes.

\subsubsection{Adjusted Associations between Other Independent Variables and CAD Hospitalization}

Appendix 4 displays the AORs and 95\% CI from the adjusted GEE models with IPTW on adherence for other independent variables. Other variables, such as cancer diagnosis categories, cancer stage, and cancer treatment were significantly associated with CAD-related hospitalization categories. Compared to men with prostate cancer, women with breast cancer $[\mathrm{AOR}=1.18 ; 95 \% \mathrm{CI}=1.08,1.29]$, women with colorectal cancer $[\mathrm{AOR}=3.31 ; 95 \% \mathrm{CI}=3.20$, 3.42], and men with colorectal cancer [AOR $=3.78$; 95\% CI $=3.67,3.88]$ were more likely to have CAD-related hospitalizations. Those with cancer stage III/IV versus stage II [AOR $=1.25 ; 95 \% \mathrm{CI}=1.15,1.34]$ and those receiving cardiotoxic cancer treatment versus no cardiotoxic cancer treatment $[\mathrm{AOR}=1.31 ; 95 \% \mathrm{CI}=1.14,1.49]$ were more likely to have CAD-related hospitalization. 
Table 3.6. Sensitivity analysis: CAD Hospitalization Identified from Primary Diagnosis Odds Ratios, Adjusted Odds Ratios, and 95\% Confidence Intervals of Medication Adherence Categories GEE Models with IPTW on CAD Hospitalization

\begin{tabular}{|c|c|c|c|c|c|c|}
\hline \multirow{2}{*}{$\begin{array}{l}\text { Medication adherence } \\
\text { categories }\end{array}$} & \multicolumn{3}{|c|}{ CAD-related hospitalization } & \multicolumn{3}{|c|}{ Other hospitalization } \\
\hline & OR/AOR & $95 \%$ CI & Sig & OR/AOR & $95 \%$ CI & Sig \\
\hline \multicolumn{7}{|c|}{ Unadjusted GEE analysis } \\
\hline No ADH 2 Rx & 2.36 & {$[2.20,2.52]$} & **** & 1.61 & {$[1.53,1.70]$} & $* * *$ \\
\hline ADH 1 of 2 Rx & 1.38 & {$[1.24,1.52]$} & *** & 1.24 & {$[1.17,1.31]$} & *** \\
\hline ADH 1 Rx & 0.76 & {$[0.56,0.95]$} & $* *$ & 1.06 & {$[0.98,1.13]$} & \\
\hline No ADH & 1.23 & {$[1.03,1.43]$} & * & 1.39 & {$[1.31,1.47]$} & $* * *$ \\
\hline \multirow{2}{*}{\multicolumn{7}{|c|}{$\begin{array}{l}\text { Reference } \\
\text { usted GEE }\end{array}$}} \\
\hline & & & & & & \\
\hline No ADH 2 Rx & 2.57 & {$[2.41,2.74]$} & $* * *$ & 1.75 & {$[1.66,1.84]$} & $* * *$ \\
\hline ADH 1 of 2 Rx & 1.42 & {$[1.27,1.57]$} & $* * *$ & 1.27 & {$[1.20,1.35]$} & $* * *$ \\
\hline ADH 1 Rx & 0.83 & {$[0.63,1.02]$} & & 1.11 & {$[1.03,1.19]$} & ** \\
\hline No ADH 1 Rx & 1.46 & {$[1.26,1.66]$} & $* * *$ & 1.58 & {$[1.49,1.67]$} & $* * *$ \\
\hline ADH 2 Rx & \multicolumn{3}{|c|}{ Reference } & \multicolumn{3}{|c|}{ Reference } \\
\hline
\end{tabular}

Note: Based on 12,096 elderly fee-for-service Medicare beneficiaries with pre-existing CAD and incident breast, colorectal, and prostate cancer diagnosis. Weights were derived using the Inverse Probability Treatment Weighting approach.

Description of medication use and adherence

ADH 2 Rx: Adherent to both statins and ACEIs/ARBs/beta-blockers;

No ADH 2 Rx: Not adherent to both statins and ACEIs/ARBs/beta-blockers;

ADH 1 of 2 Rx: Adherent to either statins or ACEIs/ARBs/beta-blockers;

ADH 1 Rx: Use of one medication class and adherent to that class;

No ADH 1 Rx: Use of one medication class and not adherent to that medication class

Asterisks represent significant differences in medication adherence groups, derived from GEE. Reference category for dependent variable is no hospitalization.

${ }^{* * *} \mathrm{p}<0.001 ; * * 0.001 \leq \mathrm{p}<0.01 ; * 0.01 \leq \mathrm{p}<0.05$.

ORs are reported for unadjusted GEE model and AORs are reported for adjusted GEE model.

Adjusted model controlled for independent variables. Time-invariant characteristics include cancer diagnosis categories, age, race/ethnicity, poverty status, high school education, concordant and discordant physical health conditions, SMI, SEER region, and county-level characteristics. Time-variant characteristics include, tobacco use, alcohol use, depression, anxiety, and routine follow-up with primary care physician and/or cardiologist, and donut hole.

Abbreviations 
AOR: Adjusted odds ratio; CAD: Coronary artery disease; CI: Confidence interval; FFS: Fee-for-service; GEE: Generalized estimating equations; OR: Odds ratio; IPTW: Inverse Probability treatment weighting; Sig:

Significance; SEER: Surveillance, Epidemiology, and End Results 


\subsection{Discussion}

To the best of our knowledge, this is the first study to examine the impact of adherence to statins and ACEIs/ARBs/beta-blockers on CAD hospitalizations in elderly FFS Medicare beneficiaries with pre-existing CAD and incident cancer diagnosis. Compared to Medicare beneficiaries with CAD and cancer who were adherent to both statins and ACEIs/ARBs/beta-blockers, those who were non-adherent to either both or single medication classes were more likely to have CAD-related hospitalization. Findings from the current study are consistent with published literature that is not specific to cancer. In these studies, non-adherence to statins and ACEIs/ARBs/beta-blockers has been reported to have negative cardiovascular health consequences, including cardiovascular events and hospitalization [94-96].

Counterintuitively, our study findings indicated that those using single medication class and adherent to that single medication class were less likely to have CAD-related hospitalization compared to those who were adherent to both medication classes. This is an unanticipated finding and plausible reasons could be that this specific population may have lower CAD severity or may be different in unobserved characteristics such as healthy eating, exercise, and maintaining normal weight. Further research is needed to understand the role of these factors in reducing CAD-related hospitalizations in the elderly Medicare beneficiaries with CAD. Interestingly, in a sensitivity analysis where CAD hospitalization was the primary diagnosis, no significant difference was observed between single medication users (adherent) and those adherent to both medication classes.

Consistent with a previous study on patients with prostate cancer diagnosis [170], we observed that CADrelated hospitalizations increased in the period immediately after cancer diagnosis for all medication adherence categories. As stated in the introduction, cardiotoxic cancer treatments can increase the risk of CAD-related hospitalizations [60-62]. This is also evident from our study findings, where those receiving cardiotoxic cancer treatment were more likely to have CAD-related hospitalizations compared to those not receiving non-cardiotoxic cancer treatment.

A meta-analysis of clinical trials showed that the prophylactic use of beta-blockers, ACEI, ARBs, or statins reduced the risk of newly developed heart failure in patients administered anthracycline [71]. We assessed the interaction between medication adherence categories and cancer treatment on CAD-related hospitalization. Our study findings revealed that the interaction effect between adherence to both medication classes and cardio-toxic cancer treatment was not statistically significant. It is possible that the established CAD diminishes the cardioprotective effect of these medications. In addition, the meta-analysis did not specifically focus on elderly population and specific cancers included in our study. Future studies that include characteristics that were not measured in the current study (example: physical activity, alternative and complementary medicine, polypharmacy) are needed to provide conclusive evidence on the lack of interaction between adherence and cardio-toxic cancer treatment.

\subsubsection{Clinical and Policy Implications}

Both CAD and cancer are life-threatening conditions and co-management of both conditions can be challenging. However, lack of effective management of CAD may not only increase CAD complications, but can also have negative consequences for cancer prognosis. Current clinical practices recommend stabilizing pre-existing 
CAD prior to initiating cancer treatment. For example, appropriate pharmacologic management (statins and betablockers) of the patient with pre-existing CAD is recommended for those undergoing cancer surgery [72]. Given the complexity of interaction between CAD and cancer, it is important to routinely monitor medication adherence in general clinical practice and provide linkages to support services that can increase medication adherence. This may warrant integration of cardiovascular care in the elderly diagnosed with incident cancer. An example of integrated care is the cardio-oncology clinics that provide interdisciplinary and integrative management approach to cancer patients with cardiovascular risks or conditions [183].

It is possible that certain CAD-related hospitalizations in our study may be readmission or preventable hospitalizations, which have policy implications. The Affordable Care Act, under Hospital Readmissions Reduction Program, provided CMS the authority to reduce payments to certain hospital readmissions that it deemed avoidable [184]. The emphasis was on Medicare readmissions related to heart failure, myocardial infarction, and pneumonia. Reducing avoidable readmissions can enhance patient quality of care and lower health care spending. In addition, based on Agency for Healthcare Research and Quality's (AHRQ) prevention quality indicators, hospitalizations related to conditions, such as angina without procedure and congestive heart failure, are considered preventable hospitalizations with good outpatient care. Therefore, it is important to implement interventions for improving medication adherence and to have integrated care for patients with pre-existing CAD and incident cancer diagnosis for reducing avoidable hospitalizations.

\subsubsection{Strengths and Limitations}

Our study findings need to be interpreted in the context of its limitations. First, the study findings cannot be generalized to all Medicare beneficiaries because the study population is restricted to those residing in SEER regions and to those with FFS Medicare plans. Further, the study population was restricted to elderly population with breast, colorectal, or prostate cancer and pre-existing CAD. The findings cannot be generalized to younger population or those with other cancer types. Second, we were not able to control for many other variables such as lifestyle health behaviors, knowledge, attitude, preferences and other factors that may affect adherence as well as hospitalizations [185]. Further, we did not look at specific cardiotoxic agents, which to some extent have overestimated cardiotoxicity measure in the study. However, we controlled for a comprehensive list of variables to examine multivariable associations. We used a proxy measure for CAD severity, which may not reflect the precise CAD severity in the population. Gold standards for measuring CAD severity, such as stress tests, are not available in Medicare database. Lastly, we used prescription claims for measuring adherence. As part D file contains only filled prescriptions, it is not known whether the patient actually used the medications or whether the patients adhered to their providers' instructions or not.

Despite these limitations, our study adds to the nascent literature on cardio-oncology and has several strengths. First, the current study included a large cohort of patients over a long period of time across a variety of providers and focused on real-world practice patterns. Second, the study used a robust repeated measure design. Third, the study used a variety of statistical methods to control for both observed and unobserved selection bias to ensure robustness of findings. 


\subsubsection{Conclusions}

Non-adherence to both statins, ACEIs/ARBs/beta blockers can increase the risk other CAD-related hospitalizations in pre-existing CAD and incident breast, colorectal, or prostate cancer. Future studies need to examine the role of patient-related factors that were not measured in this study on adherence so that strategies to improve adherence in this population can be developed. 


\section{CHAPTER 4}

\section{Impact of Incident Cancer on Short-term CAD-related Medicare Expenditures among Medicare Beneficiaries with Pre-existing Coronary Artery Disease}

\subsection{Abstract}

Objectives: To examine the association between incident cancer and short-term coronary artery disease (CAD)related Medicare expenditures among elderly with incident breast, colorectal and prostate cancer.

Methods: A retrospective observational longitudinal study was conducted among elderly Medicare fee-for-service beneficiaries with pre-existing CAD and those with incident breast (BC), colorectal (CC), or prostate (PC) cancer (N $=12,095)$ and those with no cancer (NC) $(\mathrm{N}=34,237)$. CAD-related healthcare expenditures were measured every 120 days during the one-year pre- and one-year post-index period. Due to the overlap between cancer type and sex, cancer diagnosis was grouped into: (1) women with BC; (2) women with CC; (3) women with NC; (4) men with PC; (5) men with CC; and (6) men with NC. CAD-related healthcare expenditures consisted of the actual Medicare payments for inpatient, home healthcare (HHA), and outpatient services corresponding to the ICD-9-CM primary and secondary diagnosis code for CAD. The adjusted relationship between incident cancer and CAD-related expenditures was analyzed using the generalized linear mixed models (GLMM). Inverse probability treatment weights were used to account for observed group differences between cancer and non-cancer groups.

Results: Overall, CAD-related mean healthcare expenditures at pre-index period accounted for about 32.6\% - 39.5\% of total expenditures among women and 41.5\% - 46.8\% among men. In the adjusted GLMM, all cancer groups had significantly higher CAD-related healthcare expenditures compared to the non-cancer groups. Women with CC $153 \%$ higher expenditures compared to women with no cancer [ $\beta=0.93, \mathrm{P}<0.0001]$. Men with CC had 166\% higher expenditures compared to men with $\mathrm{NC}[\beta=0.98, \mathrm{P}<0.0001]$. Further, men and women with CC had 57\% and 55\% higher expenditures compared to men with PC and women with BC, respectively.

Conclusions: CAD-related Medicare expenditures were higher for those with cancer as compared to Medicare beneficiaries without cancer. Elderly with CC were particularly vulnerable for high CAD-related expenditures. These study findings make a compelling case for of designing effective programs and policies to reduce CADrelated expenditures. Close monitoring and surveillance of CC patients with CAD may be needed to prevent CADrelated events and expenditures. 


\subsection{Introduction}

In 2013, cardiovascular disease, which included coronary artery disease (CAD) and cerebrovascular disease, ranked first in healthcare expenditures with an estimated \$231.1 billion among a mutually exclusive list of 155 conditions [186]. It has also been documented that healthcare spending for services directly resulting from CAD (i.e. CAD-related expenditures such as) were higher than for other chronic conditions [187, 188]. For these individuals, CAD-related expenditures can be higher than those with only CAD [189]. Furthermore, CAD-related healthcare expenditures in the United States (US) are projected to increase by 198\% in 2030 with increase in aging population [187].

Evidence also suggests that individuals with CAD have many pre-existing conditions or due to the shared risk factor, may develop new conditions such as cancer [190]. For these individuals, CAD-related expenditures can be higher than those with only CAD [56, 189]. Specifically, CAD-related expenditures may be higher among individuals with incident cancer (and CAD) during the period immediately after cancer diagnosis because of several reasons: 1) cardiotoxicity from specific cancer treatments can exacerbate pre-existing CAD [60, 61, 72]; and 2) diagnosis of incident cancer may impede with the management of CAD, thereby increasing the risk of CAD-related complications, given that cancer is considered a dominant condition [69].

However, there is a dearth of studies on the impact of cancer on CAD-related expenditures. It is important to analyze CAD-related expenditures among Medicare beneficiaries because there are large differences in healthcare expenditures directly attributed to CAD and total expenditures of a person with CAD [187]. More specifically, published studies suggest that inpatient accounts for nearly $43 \%$ of total expenditures for cardiovascular diseases [191]. Furthermore, CAD-related expenditures may be higher among elderly due to aging. For example, Dieleman et al. reported that $65.2 \%$ of expenditures for $\mathrm{CAD}$ and related diseases were for individuals 65 years and older, suggesting that elderly individuals account for most of the expenditures for CAD care [186]. As nearly $84 \%$ of the elderly are covered by Medicare [99], payments made by Medicare for CAD are substantial, suggesting that it is important to estimate Medicare payments for CAD care. In 2012, the Medicare program paid \$273 billion for heart disease, with per-person cost of \$10,345 [192].

Estimating the extent to which incident cancer affects CAD-related expenditures can help the payers with the emerging healthcare delivery reform initiatives, which are focused on financial incentives to improve healthcare quality with lower healthcare expenditures. For example, new payment models from the Centers for Medicare and Medicaid Services (CMS) support bundled payments for episodes of care. One of the experimental initiatives will support bundled payments for clinicians providing care to patients with CAD [193]. Such models have the potential to be extended to those who develop cancer after CAD. In addition, estimating payments for CAD-related care may also help providers by reducing costs and increasing coordination across providers. For example, Medicare Access and Children's Health Insurance Program Reauthorization Act (MACRA) of 2015, a payment reform by Medicare has created alternative payment models [193], provides financial incentives for providing value-based care and penalties for poor quality of care. These models require risk adjustment of patients [194-196], and therefore, identifying who are at risk for high-cost is important. 
The present study examined the impact of cancer on CAD-related expenditures using data from a cohort of elderly fee-for-service (FFS) Medicare beneficiaries with and without cancer. We also analyzed CAD-related inpatient expenditures and outpatient expenditures. The cancer cohort consisted of elderly with incident breast, colorectal or prostate cancer. These cancers were selected as they have a higher prevalence among elderly population with [107] and pre-existing CAD is highly prevalent among the elderly diagnosed with these cancers [109-112, 114, 197].

\subsection{Methods}

\subsubsection{Conceptual Framework}

The conceptual framework was adapted based on the Andersen's Behavioral Model of Health Service Use for understanding CAD-related healthcare expenditures and a priori selection of independent variables (Figure 1.3) [124, 125]. This model posits that individual's predisposing (age, sex, race/ethnicity), enabling (marital status, prescription drug coverage, census tract, and poverty status), need factors (chronic physical and mental health conditions), personal health practices (tobacco and alcohol use), healthcare use (visits to primary care/cardiologist and adherence to CAD medications) and external environment (Surveillance, Epidemiology and End Results [SEER] region and county metro status) may influence healthcare expenditures [126].

\subsubsection{Study Design}

A retrospective observational longitudinal cohort design with a 12-month pre- and 12-month post-index period was used (Figure 2.1). For the cancer cohort, pre- and post-index periods were based on cancer diagnosis dates. For the non-cancer cohort, pre- and post-index periods were derived using pseudo-diagnosis dates, which were randomly selected from the dates of service. Each individual was observed for 48 months with 24-month baseline (for identification of CAD and baseline characteristics) period, 12-month pre-index, and 12-month post-index periods CAD-related healthcare expenditures and selected independent variables were measured repeatedly every 120 days during the pre- $\left(\mathrm{t}_{1}, \mathrm{t}_{2}\right.$, and $\left.\mathrm{t}_{3}\right)$ and post-index $\left(\mathrm{t}_{4}, \mathrm{t}_{5}\right.$, and $\left.\mathrm{t}_{6}\right)$ period, yielding a total of six repeated measures for every single individual.

\subsubsection{Data Sources}

The data were linked from different sources, including the SEER registries; Medicare claims linked with the SEER registries; 5\% non-cancer random sample of Medicare beneficiaries in SEER region; the American Community Survey (ACS), and the Area Health Resource Files (AHRF). SEER program is an epidemiologic surveillance system comprising population-based tumor registries. It collects data on all incident cases of cancer in persons residing in 18 SEER areas. SEER data also encompass information on demographic characteristics, diagnosis date, cancer site, stage, and grade, and type of surgical treatment, and radiation therapy provided (if provided) within four months of diagnosis [126]. Medicare beneficiaries without cancer were identified from the 5\% non-cancer sample of these beneficiaries living in same catchment areas as those in SEER program, randomly selected based on the last two digits of their social security number [126]. The individuals who have been reported 
to SEER with an incident cancer are removed from this 5\% random sample. The information on census-tract level poverty status and education was derived from the ACS [128]. County-level healthcare environment factors (community health clinics, Healthcare Professional Shortage Area (HPSA), and percent of cardiologists and oncologists in the county) were derived from the AHRF [129].

\subsubsection{Study Population}

The study population comprised beneficiaries with pre-existing CAD and those with incident breast, colorectal, or prostate cancer and those without cancer. CAD was identified using a validated algorithm developed by the Centers for Medicare and Medicaid Services (CMS) [150]. The algorithm used the International Classifications of Diseases $-9^{\text {th }}$ edition, Clinical Modification (ICD-9-CM) codes. Individuals with at least two inpatient, outpatient and carrier claims (clinician encounters only), or home health agency service Medicare claims with a primary or secondary diagnosis of CAD during the baseline were classified as having pre-existing CAD. Incident cancer, January 2008 through December 2011, was identified using the International Classification of Diseases for Oncology, $3^{\text {rd }}$ Edition (ICD-O-3) codes from the SEER registries. Individuals without cancer were identified from the $5 \%$ non-cancer Medicare claims from the SEER regions.

Other inclusion criteria were age 68 years or older, alive with continuous FFS Medicare Part A, B enrollment during the entire study period, continuous part $\mathrm{D}$ enrollment during the pre- and post-index period, no missing information on county, who had two or more prescriptions for either statins or two prescriptions for any or combinations of angiotensin-converting enzyme inhibitors (ACEIs), angiotensin II receptor blockers (ARBs), or beta-blockers, and total expenditures greater than zero over the two-year period (pre- and post-index period). In the non-cancer cohort, individuals with end-stage renal disease were excluded prior to diagnosis date assignments. In the cancer cohort, individuals with missing data for type and stage of cancer and those who were diagnosed with cancer through autopsy or death certificate were excluded. The selection criteria are presented in

Figure 4.1. 


\begin{tabular}{|c|c|c|c|c|}
\hline & Breast & Colorectal & Prostate & Non-cancer cohort \\
\hline & $\downarrow$ & $\downarrow$ & $\downarrow$ & $\downarrow$ \\
\hline Cancer cases from Jan 1, 2008 to Dec 31, 2011 & $N=142,177$ & $N=103,987$ & $N=169,453$ & - \\
\hline Primary/incident cancer only, not diagnosed at autopsy/death & $N=108,619$ & $N=74,359$ & $N=139,689$ & - \\
\hline$\geq 68$ years of age at index date & $N=60,089$ & $N=49,721$ & $N=78,011$ & $N=329,250$ \\
\hline Known stage of cancer & $N=56,761$ & $N=45,440$ & $N=70,273$ & - \\
\hline No continuous $\mathrm{HMO}$ enrollment throughout study period & $N=35,167$ & $N=22,581$ & $N=45,184$ & $N=250.033$ \\
\hline Continuous Part A and B enrollment throughout study period & $\mathrm{N}=31,885$ & $\mathrm{~N}=19,957$ & $N=38,103$ & $N=206,218$ \\
\hline Continuous Part D enrollment throughout study period & $N=16,014$ & $N=9,676$ & $N=15,374$ & $N=98,118$ \\
\hline CAD diagnosis within 24 mo period, 12 mo before index date & $N=4,575$ & $N=3,490$ & $N=5,746$ & $N=46,297$ \\
\hline Excluding those with total expenditures $\leq 0$ for study period & $N=4,006$ & $N=3,104$ & $N=4,985$ & $N=34,237$ \\
\hline & & $N=12,095$ & & $N=34,237$ \\
\hline
\end{tabular}

Figure 4.1. Schematic presentation of selection criteria for study cohort.

Abbreviations - CAD: Coronary artery disease; HMO: Health Maintenance Organization 


\subsubsection{Measures}

\subsubsection{Dependent variable: CAD-related Expenditures}

CAD-related healthcare expenditures consisted of Medicare payments for inpatient, home healthcare agency (HHA), and outpatient services for CAD-related care and were measured for every 120 days $\left(t_{1}, t_{2}, t_{3}, t_{4}, t_{5}\right.$, and $\left.t_{6}\right)$. CAD-related services were identified using ICD-9-CM primary and secondary diagnosis codes for CAD [198, 199]. Prescription medication and durable medical equipment (DME) expenditures were not included because of the challenges in identifying CAD-related expenditures in DME and prescription drug claims. We also calculated total expenditures for inpatient, home healthcare (HHA), and outpatient services (obtained from outpatient and carrier claims) so that we can calculate the proportion of CAD-related expenditures to total expenditures. The outpatient claims included institutional outpatient services emergency services, laboratory tests, X-rays/other radiology, medical supplies, and preventive/screening services [200]. Carrier claims included physician services and services from other non-institutional providers, such as physician assistants and nurse practitioners [201]. The healthcare expenditures were adjusted by the Consumer Price Index for medical services [202] and expressed in 2012 constant dollars.

\subsubsection{Key independent Variable - Cancer Type and Sex}

As the study included women with breast cancer and men with prostate cancer, our key time-invariant independent variable accounted for both sex and cancer type and was categorized into six mutually exclusive groups: women with breast cancer (BCW), women with colorectal cancer (CCW), women with no cancer (NCW), men with prostate cancer (PCM), men with colorectal cancer (CCM), and men with no cancer (NCM).

\subsubsection{Other Independent Variables}

\section{Predisposing Factors}

These were time-invariant and consisted of age in years measured at index month of incident cancer diagnosis and race/ethnicity.

\section{Enabling Factors}

These factors Medicare Part D coverage gap (measured every 120 days) and included census tract-level education level and poverty status (measured at baseline). Medicare Part D coverage gap, also referred to as “donut hole” is the phase between the annual coverage limit and catastrophic coverage. Thus, Part D plans start with an annual deductible, after which Medicare pays for the prescription drugs up to an initial coverage limit with certain coinsurance associated with those payments. Prescription drugs are not covered during the period when an individual reaches the annual coverage limit and until the cost of prescription drugs reaches to catastrophic limit [156]. This creates the donut hole. 


\section{Need-based Factors}

Physical health conditions were measured at baseline and categorized into concordant (cardiac arrhythmias, congestive heart failure, diabetes, hyperlipidemia, hypertension, and stroke) and discordant (dementia, asthma, chronic obstructive pulmonary disease, arthritis, hepatitis, human immunodeficiency virus, and osteoporosis) conditions. Mental health conditions included severe mental illness (schizophrenia, bipolar disorder, and psychoses) (measured at baseline) and anxiety, and depression (measured every 120 days). For CAD severity, a proxy measure, measured at baseline, was constructed based on the CMS hierarchical condition category (HCC) classification system. Based on the CMS risk adjustment model, each of the HCC codes for CAD was assigned specific score based on the risk and severity [154], ranging from 0.231 to 0.349 . Thus, higher scores represented severe manifestations of CAD.

\section{Personal Health Care Practices}

This included tobacco use and alcohol abuse as these practices have been reported to negatively affect cardiovascular health [203, 204]. These variables were measured every 120 days.

\section{Healthcare use}

This included primary care visits, cardiologist visits, and adherence to statins and/or ACEIs, ARBs, or betablockers measured every 120 days. Adherence to these medication classes were defined as proportion of days covered (PDC). It was calculated among only those who filled at least two prescriptions for either statins or any two prescriptions of, ACEIs, ARBs, or beta-blockers. The denominator for PDC was 120 days. For those who were on both statins and ACEIs, ARBs or beta-blockers), PDC was derived by considering the number of days with both classes of medications. Individuals with PDC $\geq 80 \%$ were considered as adherent and those with PDC $<80 \%$ were considered as non-adherent [153]. Adherence was classified into five mutually exclusive groups: adherent to both statins and ACEIs/ARBs/beta-blockers, not adherent to both statins and ACEIs/ARBs/beta blockers, adherent to either statins or ACEIs/ARBs/beta-blockers, use of one medication class and adherent to that class, and use of one medication class and not adherent to that medication class.

\section{External Environment Factors}

These were time-invariant and measured at baseline. This domain included SEER region - Northeast (Connecticut, New Jersey), South (Kentucky, Louisiana, Atlanta, rural Georgia, and Greater Georgia), North-central (Detroit, Iowa), and West (San Francisco, Hawaii, New Mexico, Seattle, Utah, San Jose, Los Angeles, Greater California), county metro status (based on urban/rural continuum codes from the AHRF), and percentage of cardiologists and oncologists in the county.

In addition to the above-mentioned factors, we also controlled for time ( $\mathrm{t}_{1}$ through $\left.\mathrm{t}_{6}\right)$ to better reflect for changes with time in treatment practices.

\subsubsection{Statistical Analyses}


Unadjusted subgroup differences in time-invariant characteristics between cancer and no cancer categories by sex (six groups) were tested with chi-square statistics. Our preliminary analyses indicated that there were significant group differences in age, race/ethnicity, concordant and discordant physical health conditions, mental health conditions, SEER region, and index year between the categories. Therefore, inverse probability treatment weights (IPTW) were derived using the multinomial logistic regression on cancer and no cancer categories. These IPTWs were used when modeling expenditures to adjust for the observed differences between the categories. As seen in

Table 4.1, such weighting enabled us to balance the difference among the six groups. Table 4.2 summarizes the findings from the multinomial logistic regression used to calculate IPTW.

Healthcare expenditures have unique features such as: 1) non-negative values; 2) a large number of individuals having zero expenditures; and 3) a small number of individuals having very high expenditures. Therefore, ordinary least squares regression on expenditures is not suitable. An alternative is to transform the expenditures with natural logarithms. With log-transformed expenditures, the impact of cancer on healthcare expenditures reflect percent change in expenditures. Retransformation of the regression coefficients to original dollars involves using smearingestimators and can become very challenging [205]. Estimating expenditures with generalized linear models (GLM) with appropriate family of distribution and link function can overcome some of these problems [205].

As we measured CAD-related healthcare expenditures every 120 days during pre- and post-index period, each individual had six observations. These six observations were not independent, therefore, we used generalized linear mixed models (GLMM) with gamma distribution and log-link to analyze adjusted relationships between cancer and non-cancer groups and CAD-related healthcare expenditures. This is the most commonly used approach in previous cost analyses [206, 207]. In these GLMMs, predisposing, enabling and need factors as well as external environmental characteristics affecting healthcare expenditures were included. These models also included time as a continuous variable which varied from $t_{1}$ through $t_{6}$, and index years with 2008 as the reference group. 
Table 4.1. Unweighted and Weighted Percent of Selected Characteristics by Cancer Diagnosis

\begin{tabular}{|c|c|c|c|c|c|c|c|c|c|c|c|c|c|c|}
\hline Characteristics & $\begin{array}{c}\text { BCW } \\
(\%)\end{array}$ & $\begin{array}{c}\text { CCW } \\
(\%)\end{array}$ & $\begin{array}{c}\text { CCM } \\
(\%)\end{array}$ & $\begin{array}{c}\text { PCM } \\
(\%)\end{array}$ & $\begin{array}{c}\text { NCW } \\
(\%)\end{array}$ & $\begin{array}{c}\text { NCM } \\
(\%)\end{array}$ & Sig & $\begin{array}{c}\text { BCW } \\
\text { (wt. \%) }\end{array}$ & $\begin{array}{c}\text { CCW } \\
\text { (wt. \%) }\end{array}$ & $\begin{array}{c}\text { CCM } \\
\text { (wt. } \\
\% \text { ) } \\
\end{array}$ & $\begin{array}{c}\text { PCM } \\
\text { (wt. } \\
\% \text { ) } \\
\end{array}$ & $\begin{array}{c}\text { NCW } \\
\text { (wt. \%) }\end{array}$ & $\begin{array}{c}\text { NCM } \\
\text { (wt. } \\
\% \text { ) }\end{array}$ & Sig \\
\hline Age groups & & & & & & & $* * *$ & & & & & & & NS \\
\hline $68-70$ years & 13.8 & 9.2 & 14.7 & 21.4 & 13.7 & 22.4 & & 17.0 & 16.6 & 16.7 & 16.5 & 16.8 & 16.9 & \\
\hline $71-74$ years & 19.8 & 14.5 & 23.4 & 30.5 & 17.2 & 23.8 & & 20.7 & 20.8 & 20.7 & 20.6 & 20.6 & 20.6 & \\
\hline 75-79 years & 26.0 & 22.2 & 25.0 & 27.8 & 21.2 & 23.4 & & 22.8 & 23.5 & 23.4 & 23.0 & 23.1 & 22.9 & \\
\hline 80 years and older & 40.4 & 54.1 & 36.9 & 20.3 & 47.9 & 30.4 & & 39.5 & 39.2 & 39.2 & 39.9 & 39.5 & 39.6 & \\
\hline Race/Ethnicity & & & & & & & $* * *$ & & & & & & & NS \\
\hline Whites & 82.5 & 78.7 & 82.8 & 82.7 & 75.0 & 77.6 & & 77.5 & 77.0 & 77.1 & 76.0 & 77.2 & 77.1 & \\
\hline African Americans & 9.4 & 11.2 & 4.9 & 7.6 & 10.4 & 5.7 & & 8.7 & 8.8 & 8.7 & 9.0 & 8.7 & 8.7 & \\
\hline Hispanics & 2.9 & 2.3 & 2.1 & 2.6 & 4.3 & 4.0 & & 3.7 & 3.6 & 4.1 & 4.2 & 3.9 & 4.0 & \\
\hline Others & 5.2 & 7.8 & 10.2 & 7.1 & 10.3 & 12.7 & & 10.0 & 10.6 & 10.0 & 10.8 & 10.2 & 10.2 & \\
\hline Concordant PHC & & & & & & & $* * *$ & & & & & & & NS \\
\hline Yes & 95.5 & 96.4 & 95.4 & 94.6 & 94.0 & 92.4 & & 93.8 & 93.4 & 94.0 & 94.3 & 93.9 & 93.9 & \\
\hline No & 4.5 & 3.6 & 4.6 & 5.4 & 6.0 & 7.6 & & 6.2 & 6.6 & 6.0 & 5.7 & 6.1 & 6.1 & \\
\hline Discordant PHC & & & & & & & $* * *$ & & & & & & & \\
\hline Yes & 48.1 & 51.4 & 37.5 & 31.7 & 50.1 & 33.9 & & 43.2 & 43.9 & 43.4 & 43.9 & 43.5 & 43.8 & \\
\hline No & 51.9 & 48.6 & 62.5 & 68.3 & 49.9 & 66.1 & & 56.8 & 56.1 & 56.6 & 56.1 & 56.5 & 56.2 & \\
\hline MHC $^{\mathbf{a}}$ & & & & & & & $* * *$ & & & & & & & \\
\hline Yes & 13.0 & 13.1 & 8.9 & 5.7 & 15.2 & 9.3 & & 12.1 & 12.6 & 12.4 & 12.6 & 12.2 & 12.6 & \\
\hline No & 87.0 & 86.9 & 91.1 & 94.3 & 84.8 & 90.7 & & 87.9 & 87.4 & 87.6 & 87.4 & 87.8 & 87.4 & \\
\hline SEER Region & & & & & & & $* * *$ & & & & & & & NS \\
\hline Northeast & 24.1 & 26.3 & 24.0 & 20.0 & 22.7 & 18.9 & & 21.9 & 21.6 & 21.7 & 20.1 & 21.6 & 21.7 & \\
\hline South & 25.1 & 25.4 & 22.2 & 24.6 & 25.6 & 23.8 & & 24.9 & 24.5 & 24.7 & 25.2 & 24.7 & 24.5 & \\
\hline North-central & 13.9 & 14.5 & 12.8 & 14.2 & 11.8 & 10.5 & & 12.1 & 11.5 & 12.1 & 12.8 & 12.0 & 11.9 & \\
\hline West & 36.9 & 33.8 & 41.0 & 41.1 & 39.9 & 46.8 & & 41.1 & 42.3 & 41.5 & 41.9 & 41.6 & 41.9 & \\
\hline Index year & & & & & & & $* *$ & & & & & & & NS \\
\hline 2008 & 24.0 & 25.1 & 24.9 & 24.6 & 23.9 & 23.4 & & 24.1 & 25.9 & 23.7 & 22.8 & 23.9 & 24.7 & \\
\hline 2009 & 24.5 & 23.9 & 23.8 & 25.1 & 23.8 & 24.7 & & 23.8 & 23.7 & 24.9 & 23.6 & 24.2 & 24.2 & \\
\hline 2010 & 25.4 & 25.6 & 25.3 & 23.8 & 26.1 & 24.8 & & 26.0 & 25.7 & 25.6 & 25.5 & 25.4 & 25.2 & \\
\hline 2011 & 26.1 & 25.3 & 25.9 & 26.5 & 26.3 & 27.1 & & 26.2 & 24.7 & 25.7 & 28.1 & 26.6 & 25.9 & \\
\hline
\end{tabular}

Note: Based on 46,332 elderly fee-for-service Medicare beneficiaries with pre-existing CAD between 2008 and 2011 (12,095 cancer cohort and 34,237 non-

cancer cohort).

Individuals with incident breast, colorectal, and prostate cancer were derived from the SEER registries and individuals with no cancer were derived from the 5\% non-cancer sample from the SEER region. Weights were derived using the Inverse Probability Treatment Weights approach. 
Asterisks represent significant differences in time-invariant patient-level characteristics based on chi-square tests.

$* * * \mathrm{p}<0.001 ; * * 0.001 \leq \mathrm{p}<0.01 ; * 0.01 \leq \mathrm{p}<0.05$. Column percentages are reported.

Time-invariant characteristics include cancer diagnosis categories, age, race/ethnicity, poverty status, high school education, concordant and discordant physical health conditions, SMI, SEER region, and county-level characteristics. Time-variant characteristics include, tobacco use, alcohol use, depression, anxiety, and routine follow-up with primary care physician and/or cardiologist, and donut hole.

${ }^{a}$ Concordant physical health conditions consisted of diabetes, hyperlipidemia, hypertension, stroke, cardiac arrhythmia, and congestive heart failure. Discordant physical health conditions consisted of arthritis, asthma, chronic obstructive pulmonary disease, osteoporosis, dementia, human immunodeficiency virus, and hepatitis. Mental health conditions include anxiety, depression, SMI (schizophrenia, bipolar disorder, and psychoses)

\section{Abbreviations}

BCW: Women with breast cancer; CAD: Coronary artery disease; CCM: Men with colorectal cancer; CCW: Women with colorectal cancer; FFS: Fee-forservice; MHC: Mental health conditions; NCM: Men with no cancer; NCW: Women with no cancer; PCM: Men with prostate cancer; PHC: Physical health conditions; SEER: Surveillance, Epidemiology and End Results; SMI: Severe mental illness; Wt.: Weighted 
Table 4.2. Multinomial Logistic Regression Models used to derive IPTW

\begin{tabular}{|c|c|c|c|c|c|c|c|c|c|c|c|c|c|c|c|}
\hline \multirow[t]{2}{*}{ Characteristics } & \multicolumn{3}{|c|}{ BCW } & \multicolumn{3}{|c|}{ CCW } & \multicolumn{3}{|c|}{ CCM } & \multicolumn{3}{|c|}{ PCM } & \multicolumn{3}{|c|}{ NCW } \\
\hline & OR & $95 \% \mathrm{CI}$ & Sig & OR & $95 \% \mathrm{CI}$ & Sig & OR & $95 \% \mathrm{CI}$ & Sig & OR & $95 \% \mathrm{CI}$ & Sig & OR & $95 \% \mathrm{CI}$ & Sig \\
\hline 68-70 years & 0.48 & {$[0.43,0.54]$} & $* * *$ & 0.24 & {$[0.20,0.28]$} & $* * *$ & 0.56 & {$[0.47,0.67]$} & $* * *$ & 1.45 & {$[1.31,1.60]$} & $* * *$ & 0.39 & {$[0.37,0.42]$} & $* * *$ \\
\hline 71-74 years & 0.66 & {$[0.60,0.73]$} & $* * *$ & 0.36 & {$[0.31,0.42]$} & $* * *$ & 0.84 & {$[0.73,0.98]$} & $*$ & 1.94 & {$[1.77,2.13]$} & $* * *$ & 0.47 & {$[0.45,0.51]$} & $* * *$ \\
\hline $\begin{array}{l}75-79 \text { years } \\
80 \text { years and older }\end{array}$ & $\begin{array}{l}0.87 \\
\text { Ref }\end{array}$ & {$[0.80,0.96]$} & $* *$ & 0.55 & {$[0.49,0.63]$} & $* * *$ & 0.91 & {$[0.78,1.05]$} & & 1.81 & {$[1.64,1.99]$} & $* * *$ & 0.59 & {$[0.56,0.63]$} & $* * *$ \\
\hline Race/Ethnicity & & & & & & & & & & & & & & & \\
\hline African Americans & 1.51 & {$[1.32,1.72]$} & $* * *$ & 1.93 & {$[1.62,2.29]$} & $* * *$ & 0.80 & {$[0.62,1.04]$} & & 1.21 & {$[1.06,1.38]$} & $* *$ & 1.87 & {$[1.71,2.05]$} & $* * *$ \\
\hline Hispanics & 0.7 & {$[0.57,0.86]$} & $* * *$ & 0.59 & {$[0.42,0.83]$} & $* *$ & 0.51 & {$[0.35,0.74]$} & $* * *$ & 0.65 & {$[0.54,0.80]$} & $* * *$ & 1.13 & {$[1.01,1.27]$} & $*$ \\
\hline $\begin{array}{l}\text { Others } \\
\text { Whites }\end{array}$ & $\begin{array}{l}0.41 \\
\text { Ref }\end{array}$ & {$[0.35,0.48]$} & $* * *$ & 0.72 & {$[0.59,0.88]$} & $* *$ & 0.78 & {$[0.64,0.95]$} & $*$ & 0.52 & {$[0.46,0.59]$} & $* * *$ & 0.93 & {$[0.86,1.00]$} & \\
\hline Concordant PHC & & & & & & & & & & & & & & & \\
\hline $\begin{array}{l}\text { Yes } \\
\text { No }\end{array}$ & $\begin{array}{l}1.46 \\
\text { Ref }\end{array}$ & {$[1.24,1.73]$} & $* * *$ & 1.69 & {$[1.29,2.20]$} & $* * *$ & 1.61 & {$[1.25,2.09]$} & $* * *$ & 1.54 & {$[1.34,1.78]$} & $* * *$ & 1.00 & {$[0.92,1.09]$} & \\
\hline Discordant PHC & & & & & & & & & & & & & & & \\
\hline $\begin{array}{l}\text { Yes } \\
\text { No }\end{array}$ & $\begin{array}{l}1.68 \\
\text { Ref }\end{array}$ & {$[1.56,1.81]$} & $* * *$ & 1.82 & {$[1.64,2.02]$} & $* * *$ & 1.13 & {$[1.01,1.27]$} & $*$ & 0.96 & {$[0.89,1.03]$} & & 1.75 & {$[1.67,1.84]$} & $* * *$ \\
\hline MHC & & & & & & & & & & & & & & & \\
\hline $\begin{array}{l}\text { Yes } \\
\text { No }\end{array}$ & $\begin{array}{l}1.20 \\
\text { Ref }\end{array}$ & {$[1.07,1.34]$} & $* *$ & 1.19 & {$[1.02,1.39]$} & $*$ & 0.89 & {$[0.73,1.09]$} & & 0.58 & {$[0.50,0.66]$} & $* * *$ & 1.48 & {$[1.37,1.59]$} & $* * *$ \\
\hline SEER Region & & & & & & & & & & & & & & & \\
\hline Northeast & 1.31 & {$[1.19,1.45]$} & $* * *$ & 1.58 & {$[1.38,1.81]$} & $* * *$ & 1.32 & {$[1.14,1.54]$} & $* * *$ & 1.09 & {$[0.99,1.20]$} & & 1.27 & {$[1.20,1.36]$} & $* * *$ \\
\hline South & 1.12 & {$[1.02,1.24]$} & $*$ & 1.34 & {$[1.16,1.54]$} & $* * *$ & 1.02 & {$[0.87,1.18]$} & & 0.99 & {$[0.91,1.08]$} & & 1.24 & {$[1.16,1.31]$} & $* * *$ \\
\hline $\begin{array}{l}\text { North-central } \\
\text { West }\end{array}$ & $\begin{array}{l}1.41 \\
\text { Ref }\end{array}$ & {$[1.25,1.58]$} & $* * *$ & 1.68 & {$[1.42,1.98]$} & $* * *$ & 1.30 & {$[1.08,1.56]$} & $* *$ & 1.35 & {$[1.22,1.51]$} & $* * *$ & 1.26 & {$[1.17,1.36]$} & $* * *$ \\
\hline Index year & & & & & & & & & & & & & & & \\
\hline 2008 & 0.93 & {$[0.84,1.04]$} & & 0.91 & {$[0.78,1.05]$} & & 1.02 & {$[0.87,1.20]$} & & 1.12 & {$[1.02,1.23]$} & $*$ & 0.89 & {$[0.83,0.95]$} & $* * *$ \\
\hline 2009 & 0.95 & {$[0.86,1.05]$} & & 0.89 & {$[0.77,1.03]$} & & 0.94 & {$[0.80,1.10]$} & & 1.06 & {$[0.96,1.16]$} & & 0.90 & {$[0.84,0.96]$} & $* * *$ \\
\hline $\begin{array}{l}2010 \\
2011\end{array}$ & $\begin{array}{l}0.97 \\
\text { Ref }\end{array}$ & {$[0.87,1.07]$} & & 0.94 & {$[0.82,1.09]$} & & 0.98 & {$[0.84,1.14]$} & & 0.97 & {$[0.89,1.07]$} & & 0.98 & {$[0.92,1.05]$} & \\
\hline
\end{tabular}

Note: Based on 46,332 elderly fee-for-service Medicare beneficiaries with pre-existing CAD between 2008 and 2011 (12,095 cancer cohort and 34,237 noncancer cohort).

Individuals with incident breast, colorectal, and prostate cancer were derived from the SEER registries and individuals with no cancer were derived from the 5\% non-cancer sample from the SEER region. Weights were derived using the Inverse Probability Treatment Weights approach. 
Asterisks represent significant differences in patient-level characteristics. NCM is the reference category for dependent variable.

$* * * \mathrm{p}<0.001 ; * * 0.001 \leq \mathrm{p}<0.01 ; * 0.01 \leq \mathrm{p}<0.05$.

$\underline{\text { Abbreviations }}$

BCW: Women with breast cancer; CAD: Coronary artery disease; CCM: Men with colorectal cancer; CCW: Women with colorectal cancer; FFS: Fee-forservice; NCM: Men with no cancer; NCW: Women with no cancer; PCM: Men with prostate cancer; SEER: Surveillance, Epidemiology and End Results; Wt.: Weighted 


\subsection{Results}

\subsubsection{Characteristics of the Study Population before and after IPTW Adjustment}

The study cohort comprised 46,332 elderly FFS Medicare beneficiaries with pre-existing CAD (12,095 with cancer and 34,237 without cancer). Table 4.1 summarizes the characteristics of cancer diagnosis categories before and after IPTW adjustment. Prior to adjustment, there were statistically significant differences in age, race/ethnicity, concordant physical conditions, discordant physical conditions, mental health conditions, SEER region, and index year. After adjusting with IPTW, there were no significant differences in these variables between the cancer diagnosis categories.

Table 4.3 summarizes the mean CAD-related and total expenditures during pre- and post-index period. For purposes of this table, expenditures for $t_{1}, t_{2}$, and $t_{3}$ were aggregated and represented pre-index period expenditures and expenditures for $t_{4}, t_{5}$, and $t_{6}$ were aggregated to represent post-index period. The average CAD-related expenditures ranged from \$3,423 (BCW) to 7,022 (PCM). For all groups, the post-index period expenditures were higher than the pre-index periods. CAD-related expenditures in the post-index period were approximately 3 times higher for CCW and CCM, 2 times for BCW, and 1.5 times for PCM. The post-index CAD-related expenditures for NCM and NCW, in comparison, were similar to those in the pre-index period.

The proportion of CAD-related expenditures ranged from 32.6\% (BCW) to $45.8 \%$ (NCM) during the pre-index period and $20.8 \%$ (BCW) to $46.1 \%$ (NCM) during post-index period. Among CCM, the proportion of CAD-related expenditures was the highest during the pre-index period (46.8\%) and second highest during post-index period (40.5\%). 
Table 4.3. Mean and Standard Deviation of Coronary Artery Disease-related Expenditures by Cancer and Non-Cancer Adjusted with IPTW

\begin{tabular}{|c|c|c|c|c|c|c|}
\hline & \multicolumn{3}{|c|}{ Pre-index } & \multicolumn{3}{|c|}{ Post-index } \\
\hline & $\begin{array}{c}\text { Total } \\
\text { Wt. Mean } \\
\text { (SD) } \dagger \\
\end{array}$ & $\begin{array}{c}\text { CAD-related } \\
\text { Wt. Mean } \\
\text { (SD) } \dagger \\
\end{array}$ & $\%$ CAD-related $\ddagger$ & $\begin{array}{c}\text { Total } \\
\text { Wt. Mean } \\
\text { (SD) } \dagger \\
\end{array}$ & $\begin{array}{c}\text { CAD-related } \\
\text { Wt. Mean (SD) } \dagger\end{array}$ & $\%$ CAD-related $\ddagger$ \\
\hline BCW & $\begin{array}{c}10,493 \\
(18,644)\end{array}$ & $\begin{array}{c}3,423 \\
(10,504)\end{array}$ & $32.6 \%$ & $\begin{array}{c}29,596 \\
(27,749)\end{array}$ & $\begin{array}{c}6,154 \\
(14,419)\end{array}$ & $20.8 \%$ \\
\hline CCW & $\begin{array}{c}14,504 \\
(27,138)\end{array}$ & $\begin{array}{c}5,722 \\
(15,071)\end{array}$ & $39.5 \%$ & $\begin{array}{c}55,895 \\
(45,829)\end{array}$ & $\begin{array}{l}18,916 \\
(27,102)\end{array}$ & $33.8 \%$ \\
\hline NCW & $\begin{array}{c}12,099 \\
(22,579)\end{array}$ & $\begin{array}{c}4,662 \\
(13,670)\end{array}$ & $38.5 \%$ & $\begin{array}{c}10,656 \\
(22,379)\end{array}$ & $\begin{array}{c}4,164 \\
(13,370)\end{array}$ & $39.1 \%$ \\
\hline CСM & $\begin{array}{l}17,548 \\
(31,452)\end{array}$ & $\begin{array}{c}8,220 \\
(19,298)\end{array}$ & $46.8 \%$ & $\begin{array}{c}56,849 \\
(48,323)\end{array}$ & $\begin{array}{c}23,021 \\
(28,781)\end{array}$ & $40.5 \%$ \\
\hline PCM & $\begin{array}{l}11,089 \\
(18,689)\end{array}$ & $\begin{array}{c}4,603 \\
(12,063)\end{array}$ & $41.5 \%$ & $\begin{array}{l}28,040 \\
(27,099)\end{array}$ & $\begin{array}{c}7,022 \\
(16,316)\end{array}$ & $25.0 \%$ \\
\hline NCM & $\begin{array}{c}13,943 \\
(26,758)\end{array}$ & $\begin{array}{c}6,380 \\
(16,668)\end{array}$ & $45.8 \%$ & $\begin{array}{c}11,659 \\
(26,681)\end{array}$ & $\begin{array}{c}5,377 \\
(15,594)\end{array}$ & $46.1 \%$ \\
\hline
\end{tabular}

Note: Based on 46,332 elderly fee-for-service Medicare beneficiaries with pre-existing CAD between 2008 and 2011 (12,095 cancer cohort and 34,237 noncancer cohort).

Individuals with incident breast, colorectal, and prostate cancer were derived from the SEER registries and individuals with no cancer were derived from the 5\% non-cancer sample from the SEER region. Weights were derived using the Inverse Probability Treatment Weights approach.

$\dagger$ All expenditures reported in 2012 USD, $\ddagger$ Percentage CAD-related $=\frac{\text { CAD-related expenditures }}{\text { Total expenditures }} X 100$

\section{Abbreviations}

BCW: Women with breast cancer; CAD: Coronary artery disease; CCM: Men with colorectal cancer; CCW: Women with colorectal cancer; FFS: Fee-forservice; IPTW: Inverse Probability Treatment Weights; NCM: Men with no cancer; NCW: Women with no cancer; PCM: Men with prostate cancer; SEER: Surveillance, Epidemiology and End Results, Wt.: Weighted 


\subsubsection{CAD-related Expenditures over time by Cancer and Non-Cancer Status}

Figure 4.2 displays CAD-related expenditures over time for cancer and non-cancer groups. When time was included as an adjustor, the time coefficient was positive across all cancer group, suggesting that CAD-related expenditures increased over time for all cancer groups. However, no such differences were observed for non-cancer groups.

\subsubsection{Adjusted Relationships between Cancer and CAD-related Healthcare Expenditures}

Beta coefficient, standard error (SE), percent change, from the adjusted GLMM with gamma distribution and log link are presented in Table 4.4 (total CADrelated expenditures) and Table 4.5 (inpatient and outpatient CAD-related expenditures). Because the cancer and non-cancer groups were grouped by sex, we present CAD-related expenditures with the following contrasts: cancer versus no cancer, women versus men and cancer type (breast cancer versus women with colorectal cancer and prostate cancer versus men with colorectal cancer).

\subsubsection{Cancer versus No cancer and CAD-related Healthcare Expenditures}

After controlling for all the independent variables and time, mentioned in the methods section, and with NCW as the reference group, both BCW and CCW groups had 14\% and 151\% higher expenditures, respectively. With NCM as the reference group, both CCM and PCM had 164\% and 12\% higher expenditures, respectively.

With NCW as the reference group, CCW had 239\% higher inpatient CAD-related expenditures. There was no significant difference between BCW and NCW for inpatient CAD-related expenditures. However, compared to NCW, both BCW and CCW had 54\% and 48\% significantly higher outpatient CAD-related expenditures, respectively.

Similarly, with NCM as the reference group, CCM had 249\% significantly higher inpatient CAD-related expenditures compared to NCM. There was no significant difference between PCM and NCM for inpatient CAD-related expenditures. However, compared to NCM, both CCM and PCM had 51\% and 31\% significantly higher outpatient CAD-related expenditures, respectively

\subsubsection{Sex and CAD-related Healthcare Expenditures}

When examined by sex, healthcare expenditures were 21\% lower among NCW compared to NCM; CCW had 25\% lower expenditures compared to CCM. Similarly, NCW and CCW had lower inpatient and outpatient expenditures compared to NCM and CCM, respectively.

\subsubsection{Cancer Type and CAD-related Healthcare Expenditures}


Healthcare expenditures were 55\% higher among CCW compared to BCW. Similarly, healthcare expenditures were 57\% higher among CCM compared to PCM. Further, CCW and CCM had 67\% and 68\% higher inpatient CAD-related expenditures compared to BCW and PCM, respectively. However, there was no significant difference between CCW and BCW as well as CCM and PCM for outpatient healthcare expenditures.
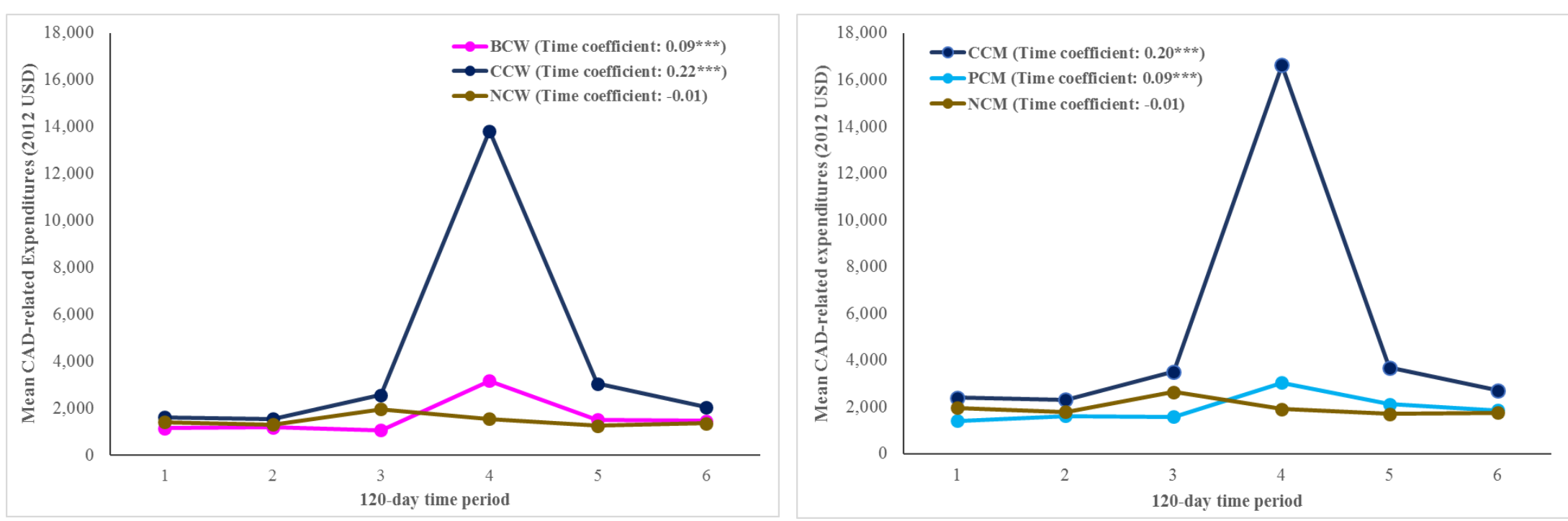

Figure 4.2. Weighted Average CAD-related Expenditures among Cancer and Non-cancer Cohorts across 120-day intervals

Note: Based on 46,332 elderly fee-for-service Medicare beneficiaries with pre-existing CAD between 2008 and 2011 (12,095 cancer cohort and 34,237 noncancer cohort)

Individuals with incident breast, colorectal, and prostate cancer were derived from the SEER registries and individuals with no cancer were derived from the 5\% non-cancer sample from the SEER region. Weights were derived using the Inverse Probability Treatment Weights approach.

Asterisks represent significant differences in cancer and non-cancer groups.

${ }^{* * *} \mathrm{p}<0.001 ; * * 0.001 \leq \mathrm{p}<0.01 ; * 0.01 \leq \mathrm{p}<0.05$

Abbreviations 
BCW: Women with breast cancer; CAD: Coronary artery disease; CCM: Men with colorectal cancer; CCW: Women with colorectal cancer; FFS: Fee-forservice; NCM: Men with no cancer; NCW: Women with no cancer; PCM: Men with prostate cancer; SEER: Surveillance, Epidemiology and End Results; Wt.: Weighted 
Table 4.4. Parameter Estimates from Generalized Linear Mixed Models on CAD-related Expenditures with IPTW

\begin{tabular}{|c|c|c|c|c|}
\hline $\begin{array}{l}\text { Cancer dx } \\
\text { categories }\end{array}$ & Coefficient & Std error & P value & \% change \\
\hline \multicolumn{5}{|c|}{ Cancer versus non-cancer differences in expenditures (Reference: $N C W)^{a}$} \\
\hline BCW & 0.13 & 0.04 & 0.002 & $14 \%$ \\
\hline CCW & 0.92 & 0.05 & 0.000 & $151 \%$ \\
\hline Intercept & 4.01 & 0.14 & & \\
\hline \multicolumn{5}{|c|}{ Cancer versus non-cancer differences in expenditures (Reference: NCM) } \\
\hline $\mathbf{C C M}$ & 0.97 & 0.05 & 0.000 & $164 \%$ \\
\hline PCM & 0.11 & 0.04 & 0.008 & $12 \%$ \\
\hline Intercept & 4.25 & 0.14 & & \\
\hline \multicolumn{5}{|c|}{ Sex differences in expenditures (Reference: $\mathrm{NCM})^{\mathrm{c}}$} \\
\hline NCW & -0.24 & 0.03 & 0.000 & $-21 \%$ \\
\hline Intercept & 4.25 & 0.14 & & \\
\hline \multicolumn{5}{|c|}{ Sex differences in expenditures (References: $\mathrm{CCM})^{\mathrm{d}}$} \\
\hline CCW & -0.29 & 0.07 & 0.000 & $-25 \%$ \\
\hline Intercept & 5.22 & 0.15 & & \\
\hline \multicolumn{5}{|c|}{ Cancer type differences in expenditures (Reference: BCW) ${ }^{\mathrm{e}}$} \\
\hline CCW & 0.79 & 0.06 & 0.000 & $55 \%$ \\
\hline Intercept & 4.93 & 0.15 & & \\
\hline \multicolumn{5}{|c|}{ Cancer type differences in expenditures (Reference: $P C M)^{f}$} \\
\hline CCM & 0.85 & 0.06 & 0.000 & $57 \%$ \\
\hline Intercept & 5.22 & 0.15 & & \\
\hline
\end{tabular}

Note: Based on 46,332 elderly fee-for-service Medicare beneficiaries with pre-existing CAD between 2008 and 2011 (12,095 cancer cohort and 34,237 non-cancer cohort).

Individuals with incident breast, colorectal, and prostate cancer were derived from the SEER registries and individuals with no cancer were derived from the $5 \%$ non-cancer sample from the SEER region. Weights were derived using the Inverse Probability Treatment Weights approach. Adjusted models controlled for time, predisposing, enabling, need, healthcare behavior, healthcare use, and environmental factors.

Significance was derived from adjusted generalized linear model with Gamma distribution and log-link. The analysis used 6 repeated measures.

$\%$ Change for GLMM was calculated as $\left(\mathrm{e}^{(\text {intercept }+\beta)}-\mathrm{e}^{\text {intercept }}\right) / \mathrm{e}^{\mathrm{intercept}}$.

${ }^{\text {a }}$ Other categories not displayed are: CCM, PCM, and NCM.

${ }^{\mathrm{b}}$ Other categories not displayed are: BCW, CCW, and NCW.

${ }^{\mathrm{C}}$ Other categories not displayed are: BCW, CCW, CCM, and PCM.

${ }^{\mathrm{d}}$ Other categories not displayed are: BCW, PCM, NCW, and NCM.

${ }^{\mathrm{e}}$ Other categories not displayed are: CCM, PCM, NCW, and NCM.

${ }^{\mathrm{f}}$ Other categories not displayed are: BCW, CCW, NCW, and NCM. 


\section{Abbreviations}

BCW: Women with breast cancer; CAD: Coronary artery disease; CCM: Men with colorectal cancer; CCW:

Women with colorectal cancer; FFS: Fee-for-service; GLMM: Generalized linear mixed models; IPTW: Inverse

Probability Treatment Weights; NCM: Men with no cancer; NCW: Women with no cancer; PCM: Men with prostate cancer; SE: Standard error; SEER: Surveillance, Epidemiology and End Results; Wt.: Weighted 
Table 4.5. Parameter Estimates from Generalized Linear Mixed Models on CAD-related Expenditures with IPTW

\begin{tabular}{|c|c|c|c|c|c|c|c|c|}
\hline \multirow{2}{*}{ Cancer $d x$ categories } & \multicolumn{4}{|c|}{ Inpatient CAD-related expenditures } & \multicolumn{4}{|c|}{ Outpatient CAD-related expenditures } \\
\hline & Coefficient & Std error & P value & Change & Coefficient & Std error & P value & Change \\
\hline \multicolumn{9}{|c|}{ Cancer versus non-cancer differences in expenditures (Reference: NCW) ${ }^{a}$} \\
\hline BCW & 0.11 & 0.08 & 0.15 & $12 \%$ & 0.43 & 0.04 & 0.000 & $54 \%$ \\
\hline $\mathbf{C C W}$ & 1.22 & 0.08 & 0.000 & $239 \%$ & 0.39 & 0.05 & 0.000 & $48 \%$ \\
\hline Intercept & 2.36 & 0.20 & & & 3.13 & 0.12 & & \\
\hline \multicolumn{9}{|c|}{ Cancer versus non-cancer differences in expenditures (Reference: NCM) ${ }^{\mathbf{b}}$} \\
\hline CCM & 1.25 & 0.08 & 0.000 & $249 \%$ & 0.41 & 0.06 & 0.000 & $51 \%$ \\
\hline PCM & 0.10 & 0.07 & 0.152 & $11 \%$ & 0.27 & 0.04 & 0.000 & $31 \%$ \\
\hline Intercept & 2.71 & 0.20 & & & 3.47 & 0.11 & & \\
\hline \multicolumn{9}{|c|}{ Sex differences in expenditures (Reference: NCM) ${ }^{c}$} \\
\hline NCW & -0.35 & 0.06 & 0.000 & $-30 \%$ & -0.35 & 0.03 & 0.000 & $-30 \%$ \\
\hline Intercept & 2.71 & 0.20 & & & 3.47 & 0.11 & & \\
\hline \multicolumn{9}{|c|}{ Sex differences in expenditures (Reference: CCM) ${ }^{\mathbf{d}}$} \\
\hline $\mathbf{C C W}$ & -0.38 & 0.10 & 0.000 & $-32 \%$ & -0.37 & 0.07 & 0.000 & $-31 \%$ \\
\hline Intercept & 3.96 & 0.21 & & & 3.89 & 0.13 & & \\
\hline \multicolumn{9}{|c|}{ Cancer type differences in expenditures (Reference: BCW) } \\
\hline $\mathbf{C C W}$ & 1.11 & 0.1 & 0.000 & $67 \%$ & -0.04 & 0.06 & 0.522 & $-4 \%$ \\
\hline Intercept & 3.58 & 0.21 & & & 3.52 & 0.13 & & \\
\hline \multicolumn{9}{|c|}{ Cancer type differences in expenditures (Reference: PCM) ${ }^{\mathrm{f}}$} \\
\hline CCM & 1.14 & 0.09 & 0.000 & $68 \%$ & 0.14 & 0.09 & 0.056 & $13 \%$ \\
\hline Intercept & 3.96 & 0.21 & & & 3.89 & 0.13 & & \\
\hline
\end{tabular}

Note: Based on 46,332 elderly fee-for-service Medicare beneficiaries with pre-existing CAD between 2008 and 2011 (12,095 cancer cohort and 34,237 noncancer cohort).

Individuals with incident breast, colorectal, and prostate cancer were derived from the SEER registries and individuals with no cancer were derived from the $5 \%$ non-cancer sample from the SEER region. Weights were derived using the Inverse Probability Treatment Weights approach. Adjusted models controlled for time, predisposing, enabling, need, healthcare behavior, healthcare use, and environmental factors.

${ }^{\mathrm{a}}$ Other categories not displayed are: CCM, PCM, and NCM.

bother categories not displayed are: BCW, CCW, and NCW.

'Other categories not displayed are: BCW, CCW, CCM, and PCM.

${ }^{\mathrm{d}}$ Other categories not displayed are: BCW, PCM, NCW, and NCM.

e Other categories not displayed are: CCM, PCM, NCW, and NCM. 
fOther categories not displayed are: BCW, CCW, NCW, and NCM.

Significance was derived from adjusted generalized linear model with Gamma distribution and log-link. The analysis used 6 repeated measures.

$\%$ Change for GLMM was calculated as $\left(\mathrm{e}^{(\text {intercept }+\beta)}-\mathrm{e}^{\text {intercept }}\right) / \mathrm{e}^{\text {intercept }}$.

$\underline{\text { Abbreviations }}$

BCW: Women with breast cancer; CAD: Coronary artery disease; CCM: Men with colorectal cancer; CCW: Women with colorectal cancer; FFS: Fee-forservice; IPTW: Inverse Probability Treatment Weights; NCM: Men with no cancer; NCW: Women with no cancer; PCM: Men with prostate cancer; SEER: Surveillance, Epidemiology and End Results; Wt.: Weighted 


\subsubsection{Relationship between Other Independent Variables and Expenditures}

Table 4.6 displays regression coefficient, standard errors, and $P$ values of other independent variables from the adjusted GLMM models. Factors associated with significantly increased expenditures included age $\geq 80$ years, concordant and discordant physical health conditions, mental health conditions, higher CAD severity, tobacco use, alcohol use, those with primary care physician or cardiologist visit, and those not adherent to one or both medication classes (for those using both medication classes). 
Table 4.6. Adjusted Relationship between Other Characteristics and CAD-related Expenditures, Repeated Measures Regressions with IPTW

\begin{tabular}{|c|c|c|c|}
\hline Characteristics & $\beta$ & SE & P value \\
\hline \multicolumn{4}{|c|}{ Predisposing factors } \\
\hline \multicolumn{4}{|l|}{ Age groups (in years) } \\
\hline $68-70$ & -0.50 & 0.04 & 0.000 \\
\hline $71-74$ & -0.48 & 0.04 & 0.000 \\
\hline $75-79$ & -0.35 & 0.03 & 0.000 \\
\hline$\geq 80$ & Ref & & \\
\hline \multicolumn{4}{|l|}{ Race/Ethnicity } \\
\hline Whites & 0.33 & 0.05 & 0.000 \\
\hline African Americans & 0.79 & 0.07 & 0.000 \\
\hline Hispanics & 0.31 & 0.08 & 0.000 \\
\hline Others & Ref & & \\
\hline \multicolumn{4}{|c|}{ Enabling factors } \\
\hline \multicolumn{4}{|l|}{ Education level } \\
\hline Less than national avg $12 \%$ & 0.06 & 0.03 & 0.031 \\
\hline GE national avg $12 \%$ & Ref & & \\
\hline \multicolumn{4}{|l|}{ Poverty level } \\
\hline Less than national avg $14.3 \%$ & -0.03 & 0.03 & 0.262 \\
\hline GE national avg $14.3 \%$ & Ref & & \\
\hline \multicolumn{4}{|l|}{ Donut hole } \\
\hline Not reached & -0.35 & 0.05 & 0.000 \\
\hline Reached & 0.06 & 0.06 & 0.337 \\
\hline Exceeded & Ref & & \\
\hline \multicolumn{4}{|c|}{ Need factors } \\
\hline \multicolumn{4}{|l|}{ Concordant $\mathrm{PHCs}^{\mathrm{a}}$} \\
\hline Yes & 0.64 & 0.08 & 0.000 \\
\hline No & Ref & & \\
\hline \multicolumn{4}{|l|}{ Discordant PHCs ${ }^{\mathrm{a}}$} \\
\hline Yes & 0.39 & 0.03 & 0.000 \\
\hline No & Ref & & \\
\hline \multicolumn{4}{|l|}{ Mental health conditions ${ }^{\mathbf{a}}$} \\
\hline Yes & 0.74 & 0.03 & 0.000 \\
\hline No & Ref & & \\
\hline CAD severity & 0.80 & 0.06 & 0.000 \\
\hline \multicolumn{4}{|c|}{ Health behavior } \\
\hline \multicolumn{4}{|l|}{ Tobacco use } \\
\hline Yes & 0.36 & 0.05 & 0.000 \\
\hline No & Ref & & \\
\hline \multicolumn{4}{|l|}{ Alcohol use } \\
\hline Yes & 0.43 & 0.11 & 0.000 \\
\hline No & Ref & & \\
\hline \multicolumn{4}{|c|}{ Healthcare use } \\
\hline \multicolumn{4}{|l|}{ Primary care visit } \\
\hline Yes & 1.23 & 0.03 & 0.000 \\
\hline No & Ref & & \\
\hline \multicolumn{4}{|l|}{ Cardiologist visit } \\
\hline Yes & 1.96 & 0.02 & 0.000 \\
\hline No & Ref & & \\
\hline \multicolumn{4}{|l|}{ Adherence to medication } \\
\hline No ADH 2 Rx & 0.55 & 0.04 & 0.000 \\
\hline ADH 1 of $2 \mathrm{Rx}$ & 0.22 & 0.03 & 0.000 \\
\hline ADH 1 Rx & -0.18 & 0.04 & 0.000 \\
\hline
\end{tabular}




\begin{tabular}{lccc} 
No ADH 1 Rx & 0.25 & 0.04 & 0.000 \\
ADH 2 Rx & Ref & & \\
\multicolumn{1}{c}{ External Environment and County-level Factors } \\
\hline SEER Region & & & \\
$\quad$ Northeast & -0.25 & 0.05 & 0.000 \\
$\quad$ South & -0.02 & 0.05 & 0.710 \\
North-central & -0.12 & 0.05 & 0.010 \\
West & Ref & & \\
Metropolitan status & & & \\
$\quad$ Metro & -0.25 & 0.04 & 0.000 \\
Non-metro & Ref & & \\
HPSA & & & \\
No shortage & -0.05 & 0.05 & 0.276 \\
Part county & 0.13 & 0.03 & 0.000 \\
Whole county & Ref & & \\
FQHC & & & \\
None & 0.04 & 0.06 & 0.510 \\
1 to 10 & 0.06 & 0.04 & 0.119 \\
GE 11 & Ref & & \\
Rural health centers & & & \\
Yes & 0.02 & 0.03 & 0.534 \\
No & Ref & & \\
Time & 0.02 & 0.01 & 0.000 \\
\hline
\end{tabular}

Note: Based on 46,332 elderly fee-for-service Medicare beneficiaries with pre-existing CAD between 2008 and 2011 (12,095 cancer cohort and 34,237 non-cancer cohort).

Individuals with incident breast, colorectal, and prostate cancer were derived from the SEER registries and individuals with no cancer were derived from the 5\% non-cancer sample from the SEER region. Weights were derived using the Inverse Probability Treatment Weights approach.

Time-invariant characteristics include cancer diagnosis categories, age, race/ethnicity, poverty status, high school education, concordant and discordant physical health conditions, SMI, SEER region, and county-level characteristics. Time-variant characteristics include, tobacco use, alcohol use, depression, anxiety, and routine follow-up with primary care physician and/or cardiologist, and donut hole.

${ }^{a}$ Concordant physical health conditions consisted of diabetes, hyperlipidemia, hypertension, stroke, cardiac arrhythmia, and congestive heart failure. Discordant physical health conditions consisted of arthritis, asthma, chronic obstructive pulmonary disease, osteoporosis, dementia, human immunodeficiency virus, and hepatitis. Mental health conditions include anxiety, depression, SMI (schizophrenia, bipolar disorder, and psychoses)

Significance was derived from adjusted generalized linear model with Gamma distribution and log-link. The analysis used 6 repeated measures.

$\underline{\text { Abbreviations }}$

ADH 2 Rx: Adherent to both statins and ACEIs/ARBs/beta-blockers; 
No ADH 2 Rx: Not adherent to both medication classes

ADH 1 of 2 Rx: Adherent to either statins or ACEIs/ARBs/beta-blockers;

ADH 1 Rx: Use of one medication class and adherent to that class;

No ADH 1 Rx: Use of one medication class and not adherent to that medication class;

Abbreviations:

ACEIs: Angiotensin-converting enzyme inhibitors; ARBs: Angiotensin II receptor blockers; CAD: Coronary artery disease; FFS: Fee-for-service; IPTW: Inverse Probability Treatment Weights; PHC: physical health conditions; SE: Standard error; SEER: Surveillance, Epidemiology and End Results; Wt.: Weighted 


\subsection{Discussion}

To date, this is amongst the first study to estimate CAD-related expenditures among cancer and non-cancer FFS Medicare beneficiaries with pre-existing CAD and analyze the relationship between incident cancer diagnosis and short-term CAD-related (inpatient and outpatient) expenditures. In general, CAD accounted for $32.6 \%$ to $46.8 \%$ of total expenditures during pre-index period and $20.8 \%$ to $46.1 \%$ during the post-index period among cancer patients. These findings suggest that a substantial portion of total expenditures accounted for care that is directly related to CAD.

Our study findings suggest that CAD-related expenditures were higher among those with cancer than those without cancer. We speculate that part of the CAD-related expenditures among patients with cancer may have resulted from cancer treatments [60, 61, 72]. Moreover, it is plausible that cancer may take precedence over CAD management and impede the recommended care for CAD, thereby increasing CAD-related complications [69] leading to higher CAD-related expenditures. For patients undergoing cancer surgery, CAD may need to be stabilized using medical management [72], further adding to the overall CAD-related expenditures among cancer patients.

A noteworthy finding is that CAD-related expenditures were highest among elderly with colorectal cancer compared to breast cancer or prostate cancer or no cancer. We observed that CAD-related expenditures were highest during 120 days immediately after colorectal cancer diagnosis. We also found that a majority of patients had advanced stage (63.0\%) colorectal cancer. Colorectal cancer treatments primarily consist of surgery and adjuvant chemotherapy (e.g., 5-fluorouracil and capecitabine) for those with Stages II- IV [67]. Taken together, these findings suggest that CAD-related expenditures may be higher among those with colorectal cancer due to chemotherapyrelated cardiotoxicity. Our preliminary study findings demonstrated that non-adherence to statins and ACEIs/ARBs/beta-blockers was significantly higher in colorectal cancer patients who had surgery (81\% in this study). Non-adherence to these medications has been previously shown to increase the risk of CAD-related complications and hospitalizations, thereby increasing inpatient expenditures [94].

CAD-related expenditures increased over time for all cancer groups. A closer examination of the study findings indicated that CAD-related expenditures were highest during the 120-day interval immediately after cancer diagnosis, suggesting that the period after cancer diagnosis may be the crucial in terms of monitoring and management of CAD.

Our study findings have significant implications for the policy makers and insurance providers. The Centers for Medicare and Medicaid Services' has rolled out demonstration projects for new bundled payment models that support payments for episodes of care [193]. Similarly, the Medicare’s shared savings program provides Accountable Care Organizations financial incentives to reduce spending and improve quality [208]. These programs use risk adjustment and also calculate expenditure benchmarks needed to provide care and get shared savings from the CMS. Collectively, our findings can help the payers to calculate these benchmark expenditures by adjusting for case-mix of Medicare beneficiaries, specifically those with pre-existing CAD and incident cancer [194-196]. In addition, our findings can help capture the episode-specific contribution of individual risk factors (age, gender, comorbidities, episode severity, etc.) to resource use similar to those implemented by the PROMETHEUS payment models [196, 209]. Currently, there are no specific payment models that account for cancer and CAD, but available 
models, such as PROMETHEUS can be tailored to the specific patterns of resource use within CAD. Therefore, it is crucial to understand the extent to which a specific cancer contributes to CAD-related expenditures to implement these models. Based on our study findings, it is apparent that resource allocation for CAD-related expenditures should be higher for those with colorectal cancer, followed by prostate and breast cancer.

We also found that CAD-related inpatient expenditures accounted for two-thirds of the overall CAD-related health care expenditures. Although CMS has imposed penalties for potential hospital readmissions and preventable hospitalizations, such as inpatient admissions for angina without procedures [210], these initiatives may not be effective in patients with complex conditions such as CAD and cancer. In this context, future research needs to focus on collaborative care models such as the patient-centered medical home (PCMH) because such models have been shown to reduce inpatient use. For example, a review focusing on patient-centered medical home (PCMH) indicated that PCMH was associated with reduction in inpatient hospital expenditures [211]; Geisinger Health System’s patient-centered medical home clinics reported 18.6\% cost savings for inpatient care, the largest contributor to the total cost savings [212].

Another noteworthy finding was that women had lower CAD-related expenditures compared to men. Chiha et al. assessed the differences in CAD severity among men and women and noted that women were more likely to have normal coronary arteries or less severe disease than age-matched men [213]. Although we controlled for severity of CAD with HCC, future research needs to explore whether the lower expenditures among women are due to sex differences in severity of CAD.

\subsubsection{Strengths and limitations}

This study adopted a longitudinal design and compared expenditures over time between cancer and non-cancer groups. We also used statistical adjustment for selection bias in observed and unobservable characteristics. The use of Medicare FFS claims data enabled us to track individuals across various providers and settings and calculate expenditures and measure variables for specific time periods. The study also adjusted a comprehensive list of factors that can influence CAD-related expenditures. The study included individuals with significant medical comorbidities.

Our study findings need to be interpreted in the context of its limitations. The study findings cannot be generalized to all Medicare beneficiaries because the study population is restricted to those residing in SEER regions and to those with FFS Medicare plans. Further, the SEER-Medicare data are not developed for research purposes and therefore have limitations associated with its use for estimating healthcare expenditures. It is possible that CAD diagnosis may be under-coded or misclassified in claims data, as these data are dependent on professional ICD coding. Therefore, there might be underestimation of CAD-related diagnosis, which in turn, may undermine CADrelated expenditures. Finally, we were not able to control for patient-level lifestyle health behaviors, knowledge, attitude, and preferences as well as number of cancer-related complications, treatment side-effects, and cost-related factors that may affect CAD-related expenditures.

\subsubsection{Conclusions}


Our study provided unique information and contributed to the nascent literature on cardio-oncology by comparing CAD-related expenditures among elderly FFS Medicare beneficiaries with and without cancer. We found that incident cancer diagnosis was associated with higher short-term CAD-related expenditures as compared to those with no cancer diagnosis. Specifically, inpatient expenditures for colorectal cancer patients were considerably higher than outpatient expenditures. This suggests that more emphasis is warranted on preventing cardiac events in the outpatient setting in order to reduce the more expensive inpatient encounters. Future studies need to explore whether the emerging payment reforms and collaborative care models can lower costs while maintaining high quality CAD care for patients with and without cancer. 


\section{Chapter 5 \\ Discussion of Findings and Research Implications}

\subsection{Summary of Findings and Discussion}

Coronary artery disease (CAD) is one of the most burdensome chronic conditions in the elderly [4, 12, 24, 25, 27]. The two key goals of long-term management of CAD are (i) to reduce symptoms and ischemia and (ii) prevent myocardial infarction and death, by lowering lipids and blood pressure [29]. Of all the risk reduction strategies, use and adherence to concomitant pharmacotherapy with statins and beta-blockers, angiotensin-converting enzyme inhibitors (ACEIs) or angiotensin II receptor blockers (ARBs) have been shown to be highly effective and has become the cornerstone of CAD management. However, adherence to concomitant pharmacotherapy can be influenced by many factors including the development of other life-threatening conditions such as cancer. The presence of CAD and incident cancer is a prototype example of AHRQ definition of a complex chronic illness "two or more chronic conditions in which each condition might influence the care of the other condition, either through a limitation on life expectancy imposed by one of the conditions or because these two conditions generally entail therapies which are likely to have interactions with each other, or thirdly wherein one of these conditions might be requiring therapies that are contraindicated by the presence of the other conditions” [73]. To date, no real-world study has assessed how incident cancer can affect adherence to concomitant pharmacotherapy and whether concomitant pharmacotherapy can minimize the negative effects of some cancer treatments on CAD-related morbidity and expenditures among individuals with CAD and incident cancer.

The subsequent presence of incident cancer among individuals with CAD is especially important for many reasons: (i) CAD and cancer have shared risk factors and unique pathophysiology; (ii) adults with CAD are more likely to develop cancer; (iii) there can be negative effects of some cancer treatments on cardiovascular health; (iv) as both CAD and cancer are life-threatening conditions, the co-management of these conditions is especially challenging; and (v) both conditions are very costly to treat and manage and such heavy financial burden has implications for payers, healthcare providers and patients.

Cardio-oncology or onco-cardiology is an emerging field and most of the existing evidence on management of heart diseases in individuals with cancer is based on the clinical expert opinion. However, there is paucity of studies on the influence of cancer diagnosis on management of CAD. The current study contributes to the nascent literature on management of CAD in patients with cancer. This study focused on 1) association between incident breast, colorectal, and prostate cancer diagnosis and adherence to statins and/or ACEIs/ARBs/beta-blockers among elderly fee-for-service (FFS) Medicare beneficiaries with pre-existing CAD; 2) the impact of non-adherence to these medication classes on short-term CAD-related hospitalizations in patients with incident cancer diagnosis; and 3) the impact of incident cancer diagnosis on short-term CAD-related inpatient and outpatient healthcare expenditures.

We observed that both men and women diagnosed with incident colorectal cancer were less likely to adhere to both statins and ACEIs/ARBs/beta-blockers compared to non-cancer women and men, even after controlling for a comprehensive list of factors. In addition, men with incident prostate cancer were less likely to adhere to both 
medication classes, but the magnitude of difference was much less compared to men with no cancer. There were no differences in adherence to both medication classes by presence of breast cancer. Taken together, these finding suggest that cancer type rather than cancer itself may affect medication adherence. Furthermore, we also found that among Medicare beneficiaries with CAD and colorectal cancer, those with late-stage cancer compared to early stage cancer and those who had surgical treatment for cancer compared to no surgical treatment were less likely to be adherent to both statins and ACEIs/ARBs/beta-blockers. These findings suggest that cancer stage and cancer treatment may affect medication adherence among colorectal cancer patients.

These findings from our study highlight the difficulty in sustaining ongoing therapy for CAD among these individuals and their vulnerability to CAD-related complications. Extant literature shows that non-adherence to statins and ACEIs/ARBs/beta-blockers has negative cardiovascular health consequences, including cardiovascular events and hospitalization [94-96]. Further evidence suggests an increase in the risk of first hospitalization for CAD during the six months after cancer diagnosis [171], perhaps due to cardiotoxicity associated with cancer treatments such as radiotherapy, chemotherapy, and hormone therapy [60-62]. We analyzed the relationship between adherence to both statins and ACEIs/ARBs/beta-blockers and CAD-related hospitalizations among the elderly Medicare beneficiaries with pre-existing CAD and incident breast, colorectal, or prostate cancer.

Compared to Medicare beneficiaries with CAD and cancer who were adherent to both statins and ACEIs/ARBs/beta-blockers, those who were non-adherent to either both or single medication classes were more likely to have CAD-related hospitalization. We also observed that CAD-related hospitalizations increased in the period immediately following cancer diagnosis for all medication adherence categories. Our findings are consistent with the published literature (not specific to cancer) that non-adherence to statins and ACEIs/ARBs/beta-blockers have negative cardiovascular health consequences [94-96].

Coronary artery disease is ranked first in healthcare expenditures, with an estimated \$231.1 billion (2013) [186]. A large proportion of these expenditures can be attributed to CAD-related hospitalizations and outpatient care [188]. For the individuals with incident cancer diagnosis, CAD-related expenditures can be higher than those with only CAD [189] as specific cancer treatments may worsen pre-existing CAD and cancer treatment may, dominantly, impede the continual management of CAD. We analyzed the impact of incident cancer on CAD-related expenditures within our study population.

Our findings suggest that CAD accounted for substantial proportion of total expenditures, i.e., ranging from $32.6 \%$ to $46.8 \%$ at pre-index period. These are similar to the findings from existing studies [186], which suggest that CAD is one of the most expensive chronic conditions. In addition, CAD-related expenditures were higher among those with cancer than those without cancer. We can speculate that higher CAD-related expenditures among patients with cancer can be due to pre-existing cardiac health, resulting from cancer treatments [60,61, 72].

Surprisingly, CAD-related expenditures were highest among elderly with colorectal cancer and significantly higher for colorectal cancer compared to breast cancer or prostate cancer. We observed that CAD-related expenditures were highest during 120 days immediately following colorectal cancer diagnosis. We also found that a majority of patients had advanced stage (63.0\%) colorectal cancer. The treatment for colorectal cancer primarily consists of surgery and adjuvant chemotherapy (e.g., 5-fluorouracil and capecitabine) for those with Stages II- IV 
[67]. It has been reported that patients receiving 5-fluorouracil have $45 \%$ risk of angina and 22\% risk of MI [67]. Taken together, these findings suggest that CAD-related expenditures may be higher among those with colorectal cancer due to chemotherapy-related cardiotoxicity, interference from cancer treatment, and/or non-adherence to CAD medications. Noticeably higher inpatient expenditures in our study for those with colorectal cancer compared to non-cancer or other cancer (breast and prostate) groups can be attributed to increase in CAD-related complications in these patients. Our study findings also demonstrated that the time coefficient was positive across all cancer groups and no such difference was observed for non-cancer groups. This suggests that CAD-related expenditures increased over time for all cancer groups. A closer examination of the study findings indicated that CAD-related expenditures were highest during the 120-day interval immediately after cancer diagnosis. This suggests that the period after cancer diagnosis is the crucial period for co-management of CAD and cancer.

Together, our study findings suggest that cancer diagnosis negatively impacts adherence to CAD pharmacotherapy. Reduction in adherence was associated with increase in CAD-related hospitalizations and subsequent increase in CAD-related expenditures. Our study findings warrant the integration of cardiovascular care in the elderly diagnosed with cancer.

\subsection{Clinical Implications of the Findings}

\subsubsection{Clinical Implications}

Both CAD and cancer are life-threatening conditions and co-management of both conditions can be challenging. Lack of effective management of CAD may not only increase CAD complications, but can also have negative consequences for cancer prognosis. Current clinical practices recommend stabilizing pre-existing CAD prior to initiating cancer treatment. For example, appropriate pharmacologic management (statins and beta-blockers) of the patient with pre-existing CAD is recommended for those undergoing cancer surgery [72]. In addition, statins and ACEIs/ARBs/beta-blockers are central to improving CAD-related health outcomes. Statins have been shown to reduce cancer-specific mortality in those with breast, colorectal, and prostate cancer [144-146, 164]. Further, medications for CAD may also reduce cancer treatment-related cardiotoxicity [71]. Given the complexity of interaction between CAD and cancer, it is important to routinely monitor medication adherence in general clinical practice and provide linkages to support services that can increase medication adherence.

Medication adherence is a multidimensional issue in which providers, patients, payers, and policy makers are inextricably linked and all of them need to be involved in improving medication adherence [165]. It may be very challenging for clinicians to implement interventions in routine practice because those successful-to-date have included multiple, heterogeneous components [141]. In this context, the recent healthcare delivery models such as the medical home may help in overcoming the challenges to coordination across different providers. It has been shown that patient-centered medical homes can improve adherence to medications among those with cardiovascular conditions [166]. Therefore, an interdisciplinary and integrated approach to cardiovascular management in the elderly diagnosed with incident cancer, such as the burgeoning cardio-oncology clinics, would improve cardiovascular outcomes [183]. 


\subsubsection{Policy Implications}

Recognition and management of CAD alongside cancer care are pivotal priorities. Our study findings have several policy implications. First, examination of adherence to statins and ACEIs/ARBs/beta-blockers among the elderly is important for policy purposes because Medicare provides coverage for almost all of the elderly in the US [99] and Medicare payments for CAD [100] and cancer care [101] are substantial. Our study findings highlight the need for practice and policy measures to increase CAD management, especially adherence to CAD pharmacotherapy among elderly diagnosed with incident colorectal cancer. However, under the FFS programs, there are no incentives to coordinate care across multiple systems, providers, and payers [141]. In this context, the new fiscal incentives by the CMS in paying physicians who provide care for patients with multiple chronic conditions and who invest time and resources to provide more coordinated and patient-centered care [161] may also promote adherence. It has to be noted that a systematic review of medication adherence among those with multiple chronic conditions reported that medication adherence interventions were "almost exclusively" conducted by the pharmacists [162]. In recognition of the critical role of pharmacists, Medicare now covers Medication Management Services (MTM) of pharmacists. Therefore, implementation of such policies among CAD patients can improve adherence [163].

Second, policy measures have also focused on reducing CAD-related hospitalizations, specifically readmission and preventable hospitalizations. The Affordable Care Act, under Hospital Readmissions Reduction Program, provided CMS the authority to reduce payments to hospital readmissions for certain conditions (heart failure, myocardial infarction, and pneumonia) that it deemed avoidable [184]. CMS has imposed penalties for potential hospital readmissions and preventable hospitalizations, such as inpatient admissions for angina without procedures [210], these initiatives may not work in patients with complex conditions such as CAD and cancer and alternative models may be needed to reduce inpatient expenditures. In addition, based on AHRQ's prevention quality indicators, hospitalizations related to conditions, such as angina without procedure and congestive heart failure, are considered preventable hospitalizations with good outpatient care. Therefore, it is important to implement policies focusing on integrated care for patients with pre-existing CAD and incident cancer diagnosis in order to reduce avoidable hospitalizations. Future research needs to focus on collaborative care models such as the patient-centered medical home because such models have been shown to reduce inpatient use. A review focusing on patient-centered medical home (PCMH) indicated that PMCH was associated with reduction in inpatient hospital expenditures [211]. For example, Geisinger Health System's patient-centered medical home clinics showed 18.6\% cost savings for inpatient care, the largest contributor to the total cost savings [212].

Third, estimating the extent to which incident cancer affects CAD-related expenditures can help the payers with the emerging healthcare delivery reform initiatives, which are focused on financial incentives to improve healthcare quality with lower healthcare expenditures. New payment models from the CMS support bundled payments to clinicians for providing care to patients with CAD [193]. Such models have the potential to be extended to those who develop cancer after CAD. In addition, estimating payments for CAD-related care may also help providers because the Medicare Access and Children’s Health Insurance Program Reauthorization Act of 2015 (MACRA; payment reform by Medicare) has created alternative payment models [193]. Similarly, the Medicare's shared savings program provides Accountable Care Organizations financial incentives to reduce spending and improve 
quality [208]. These programs use risk adjustment while also setting the expected expenditure benchmark for individuals with pre-existing CAD and incident cancer diagnosis [194-196]. For example, bundled payment model, PROMETHEUS, creates different risk adjustment models for each episode of care, where each model captures the episode-specific contribution of individual risk factors (age, gender, comorbidities, episode severity, etc.) to resource use [196, 209]. Understanding the extent to which a specific cancer contributes to CAD-related expenditures can tailor such models to the specific patterns of resource use within CAD.

\subsection{Unique Contributions of the Study}

There are several facets of our study that makes it unique. First, it fills a critical knowledge gap on the management of chronic conditions among patients with incident cancer diagnosis. It is important to recognize ways in which cancer could affect the management of pre-existing CAD in cancer patients so that patient-centered care and individualized treatment can be achieved. Second, the study findings can illuminate the complex relationship between CAD management and incident cancer. Prior studies have suggested that cardiovascular disease management is compromised in cancer patients. Third, this study is a population-based study utilizing "real-world" data. It has been pointed out by the investigators of the CLARIFY study that real-world studies on CAD are warranted because "clinical trials or registries which often have stringent exclusion criteria, and do not adequately represent populations with stable CAD (in terms of age, comorbidity, and concomitant therapy)”. This study is the next best alternative to clinical trials for understanding this relationship. Fourth, this dissertation focuses on elderly population and it is estimated that by 2050, there will be 83.7 million over the age of 65. More importantly, both $\mathrm{CAD}$ and cancer are highly prevalent in aging population. An understanding of the influence of life events such as cancer diagnosis on management of pre-existing CAD is important to the growing elderly population with these chronic conditions. Lastly, this study focuses on elderly FFS Medicare beneficiaries. Medicare is the primary payer of healthcare for elderly in the US and bears the largest share of the clinical, humanistic, and economic burden in this population. The findings from this study will have implications for the various healthcare delivery and financing reforms such as the establishment of accountable care organizations, patient-centered medical home and others undertaken by the Centers for Medicare and Medicaid Services, which is responsible for setting policy priorities and healthcare payments.

\subsection{Strengths and Limitations of the Study}

\subsubsection{Study Strengths}

The study has several strengths. First, a robust repeated measures study design was used comparing a large cohort of cancer and non-cancer cohort over a long period of time across a variety of providers. Further, the study used a variety of statistical methods to control for both observed and unobserved selection bias to ensure robustness of findings Second, Medicare FFS claims data aided in the measurement of adherence over time and followed a large cohort of patients for a long period of time across a variety of providers. Third, the study adjusted a comprehensive list of factors that can influence medication adherence. Fourth, the study included individuals with 
significant medical comorbidities, typically excluded from randomized clinical trials and examined real-world practice patterns.

\subsubsection{Study Limitations}

The study findings need to be interpreted in the context of its limitations. First, the study findings cannot be generalized to all Medicare beneficiaries because the study population is restricted to those residing in SEER regions and to those with FFS Medicare plans. It is anticipated that those with health maintenance organization plans may receive improved quality of care, hence, the study might have underestimated adherence and may have overestimated hospitalizations and expenditures by focusing only on FFS Medicare beneficiaries. Further, the study population was restricted to elderly population with breast, colorectal, or prostate cancer and pre-existing CAD. Second, a proxy measure was used for CAD severity, which may not reflect the precise CAD severity in the population. Gold standards for measuring CAD severity, such as stress tests, are not available in Medicare database. Also, with claims database, it is not possible to determine how cancer was confirmed. Third, the study did not control for patient-level lifestyle health behaviors, knowledge, attitude, and preferences as well as number of cancerrelated complications, treatment side-effects, and cost-related factors that may affect adherence as well as hospitalizations. However, a comprehensive list of variables was controlled to examine multivariable associations. Fourth, the SEER-Medicare data are not developed for research purposes and therefore have limitations associated with its use for estimating healthcare expenditures. Fifth, the study included patients who were alive during the study period, which might have underestimated expenditures as patients receiving end-of-life care are anticipated to have higher expenditures and CAD-related hospitalizations. Further, those receiving end-of-life care are anticipated to have lower adherence. Lastly, prescription claims were used for measuring adherence. As part D file contains only filled prescriptions, it is not known whether the patient actually used the medications or whether the patients they adhered to their providers' instructions or not.

\subsection{Suggestions for Future Research}

The current study has implications for further investigation. Studies need to examine whether the proven strategies of medication adherence can also be applied to improve medication adherence of multiple drug regimens.

Our study findings indicated that those using single medication class and adherent to that single medication class were less likely to have CAD-related hospitalization compared to those who were adherent to both medication classes. In addition, the interaction between medication adherence categories and cardiotoxic cancer treatment on CAD-related hospitalization was not statistically significant. Therefore, future studies are needed to provide conclusive evidence on the role of CAD severity and unobserved factors (healthy eating, exercise, alternative and complementary medicine, and polypharmacy) for the stated findings.

Our study findings indicated that CAD-related expenditures were highest during the 120-day interval immediately following cancer diagnosis. This suggests that the period after cancer diagnosis is the crucial period for co-management of CAD and cancer. This is an interesting finding and needs further research to understand the cancer-related factors that might contribute to this increase in expenditures with time in those diagnosed with cancer. 
Our study findings also indicated that those with colorectal cancer may be particularly at risk for high CAD-related healthcare expenditures. Future studies need to explore whether the emerging collaborative care models, such as medical homes, can reduce inpatient use, and consequently, CAD-related expenditures. 


\section{References}

1. National Center for Chronic Disease Prevention and Health Promotion. Heart Disease Facts. 2013. Available at: http://www.cdc.gov/heartdisease/facts.htm. Accessed March 3, 2016

2. Global Burden of Disease Study C. Global, regional, and national incidence, prevalence, and years lived with disability for 301 acute and chronic diseases and injuries in 188 countries, 1990-2013: a systematic analysis for the Global Burden of Disease Study 2013. Lancet 2015;386:743-800

3. Wilson PWF, Douglas, P.S.,. Epidemiology of coronary heart disease. 2016. Available at: http://www.uptodate.com/contents/epidemiology-of-coronary-heart-disease. Accessed March 3, 2016

4. Mozaffarian D, Benjamin EJ, Go AS, et al. Heart disease and stroke statistics--2015 update: a report from the American Heart Association. Circulation 2015;131:e29-322

5. Safford MM, Brown TM, Muntner PM, et al. Association of race and sex with risk of incident acute coronary heart disease events. JAMA 2012;308:1768-1774

6. Fang J, Shaw KM, Keenan N.L. Prevalence of Coronary Heart Disease — United States, 2006-2010 2011. Available at: http://www.cdc.gov/mmwr/pdf/wk/mm6040.pdf. Accessed February 21, 2016

7. Maas AH, Appelman YE. Gender differences in coronary heart disease. Neth Heart J 2010;18:598-602

8. Pena JM, Min JK. Coronary artery disease: Sex-related differences in CAD and plaque characteristics. Nat Rev Cardiol 2016;13:318-319

9. $\quad$ De Backer GG. The global burden of coronary heart disease. Medicographia 2009;31:343-348

10. World Health Organization. The 10 leading causes of death in the world, 2000 and 2012. 2014. Available at: http://www.who.int/mediacentre/factsheets/fs310/en/. Accessed May 27, 2016

11. Mathers CD, Loncar D. Projections of global mortality and burden of disease from 2002 to 2030. PLoS Med 2006;3:e442

12. Centers for Disease Control and Prevention. Heart Disease. 2015. Available at: http://www.cdc.gov/heartdisease/coronary_ad.htm. Accessed February 21, 2016

13. Graham G. Population-based approaches to understanding disparities in cardiovascular disease risk in the United States. Int J Gen Med 2014;7:393-400

14. Gillespie CD, Wigington C, Hong Y, et al. Coronary heart disease and stroke deaths - United States, 2009. MMWR Suppl 2013;62:157-160

15. World Health Organization. Prevention of Recurrences of Myocardial Infarction and Stroke Study. 2016. Available at:

http://www.who.int/cardiovascular_diseases/priorities/secondary_prevention/country/en/index1.html. Accessed May 27, 2016

16. Tunstall-Pedoe H, Kuulasmaa K, Amouyel P, et al. Myocardial infarction and coronary deaths in the World Health Organization MONICA Project. Registration procedures, event rates, and case-fatality rates in 38 populations from 21 countries in four continents. Circulation 1994;90:583-612

17. Maddox TM, Reid KJ, Spertus JA, et al. Angina at 1 year after myocardial infarction: prevalence and associated findings. Arch Intern Med 2008;168:1310-1316 
18. World Health Organization. Global burden of coronary heart disease. The Atlas of Heart Disease and Stroke2016

19. McKenna MT, Michaud CM, Murray CJ, et al. Assessing the burden of disease in the United States using disability-adjusted life years. Am J Prev Med 2005;28:415-423

20. Evaluation. IfHMa. GBD profile: United States. Seattle, WA2010

21. Thompson DR, Yu CM. Quality of life in patients with coronary heart disease-I: assessment tools. Health and quality of life outcomes 2003;1:42

22. Beck CA, Joseph L, Belisle P, et al. Predictors of quality of life 6 months and 1 year after acute myocardial infarction. Am Heart J 2001;142:271-279

23. Bosworth HB, Siegler IC, Brummett BH, et al. The association between self-rated health and mortality in a well-characterized sample of coronary artery disease patients. Med Care 1999;37:1226-1236

24. Merrill CT, Owens PL, Stocks C. Emergency Department Visits for Adults in Community Hospitals from Selected States, 2005: Statistical Brief \#47. Healthcare Cost and Utilization Project (HCUP) Statistical Briefs. Rockville (MD)2006

25. Greer S.A. NIA, Casper M.L. Atlas of Heart Disease Hospitalizations Among Medicare Beneficiaries. 2010. Available at: http://www.cdc.gov/dhdsp/atlas/2010_heart_atlas/. Accessed March 13, 2016

26. Heidenreich PA, Trogdon JG, Khavjou OA, et al. Forecasting the future of cardiovascular disease in the United States: a policy statement from the American Heart Association. Circulation 2011;123:933-944

27. Fihn SD, Gardin JM, Abrams J, et al. 2012 ACCF/AHA/ACP/AATS/PCNA/SCAI/STS Guideline for the diagnosis and management of patients with stable ischemic heart disease: a report of the American College of Cardiology Foundation/American Heart Association Task Force on Practice Guidelines, and the American College of Physicians, American Association for Thoracic Surgery, Preventive Cardiovascular Nurses Association, Society for Cardiovascular Angiography and Interventions, and Society of Thoracic Surgeons. J Am Coll Cardiol 2012;60:e44-e164

28. Zhao Y, Zabriski S, Bertram C. Associations between statin adherence level, health care costs, and utilization. Journal of managed care \& specialty pharmacy 2014;20:703-713

29. Cassar A, Holmes DR, Jr., Rihal CS, et al. Chronic coronary artery disease: diagnosis and management. Mayo Clin Proc 2009;84:1130-1146

30. Pedersen TR, Kjekshus J, Berg K, et al. Randomised trial of cholesterol lowering in 4444 patients with coronary heart disease: the Scandinavian Simvastatin Survival Study (4S). 1994. Atherosclerosis Supplements 2004;5:81-87

31. Heart Protection Study Collaborative G. MRC/BHF Heart Protection Study of cholesterol lowering with simvastatin in 20,536 high-risk individuals: a randomised placebo-controlled trial. Lancet 2002;360:7-22

32. Prevention of cardiovascular events and death with pravastatin in patients with coronary heart disease and a broad range of initial cholesterol levels. The Long-Term Intervention with Pravastatin in Ischaemic Disease (LIPID) Study Group. N Engl J Med 1998;339:1349-1357 
33. Sacks FM, Moye LA, Davis BR, et al. Relationship between plasma LDL concentrations during treatment with pravastatin and recurrent coronary events in the Cholesterol and Recurrent Events trial. Circulation 1998;97:1446-1452

34. Lewington S, Clarke R, Qizilbash N, et al. Age-specific relevance of usual blood pressure to vascular mortality: a meta-analysis of individual data for one million adults in 61 prospective studies. Lancet 2002;360:1903-1913

35. The Seventh Report of the Joint National Committee on Prevention, Detection, Evaluation, and Treatment of High Blood Pressure. Bethesda (MD)2004

36. Rosendorff C, Black HR, Cannon CP, et al. Treatment of hypertension in the prevention and management of ischemic heart disease: a scientific statement from the American Heart Association Council for High Blood Pressure Research and the Councils on Clinical Cardiology and Epidemiology and Prevention. Circulation 2007;115:2761-2788

37. National Cholesterol Education Program Expert Panel on Detection Evaluation Treatment of High Blood Cholesterol in Adults. Third Report of the National Cholesterol Education Program (NCEP) Expert Panel on Detection, Evaluation, and Treatment of High Blood Cholesterol in Adults (Adult Treatment Panel III) final report. Circulation 2002;106:3143-3421

38. Sacks FM, Tonkin AM, Shepherd J, et al. Effect of pravastatin on coronary disease events in subgroups defined by coronary risk factors: the Prospective Pravastatin Pooling Project. Circulation 2000;102:1893-1900

39. Lewis SJ, Moye LA, Sacks FM, et al. Effect of pravastatin on cardiovascular events in older patients with myocardial infarction and cholesterol levels in the average range. Results of the Cholesterol and Recurrent Events (CARE) trial. Ann Intern Med 1998;129:681-689

40. Miettinen TA, Pyorala K, Olsson AG, et al. Cholesterol-lowering therapy in women and elderly patients with myocardial infarction or angina pectoris: findings from the Scandinavian Simvastatin Survival Study (4S). Circulation 1997;96:4211-4218

41. Ganz DA, Kuntz KM, Jacobson GA, et al. Cost-effectiveness of 3-hydroxy-3-methylglutaryl coenzyme A reductase inhibitor therapy in older patients with myocardial infarction. Ann Intern Med 2000;132:780-787

42. Freemantle N, Cleland J, Young P, et al. beta Blockade after myocardial infarction: systematic review and meta regression analysis. BMJ 1999;318:1730-1737

43. Law MR, Morris JK, Wald NJ. Use of blood pressure lowering drugs in the prevention of cardiovascular disease: meta-analysis of 147 randomised trials in the context of expectations from prospective epidemiological studies. BMJ 2009;338:b1665

44. Tummala PE, Chen XL, Sundell CL, et al. Angiotensin II induces vascular cell adhesion molecule-1 expression in rat vasculature: A potential link between the renin-angiotensin system and atherosclerosis. Circulation 1999;100:1223-1229

45. Lonn EM, Yusuf S, Jha P, et al. Emerging role of angiotensin-converting enzyme inhibitors in cardiac and vascular protection. Circulation 1994;90:2056-2069 
46. Yusuf S, Sleight P, Pogue J, et al. Effects of an angiotensin-converting-enzyme inhibitor, ramipril, on cardiovascular events in high-risk patients. The Heart Outcomes Prevention Evaluation Study Investigators. N Engl J Med 2000;342:145-153

47. Rodrigues EJ, Eisenberg MJ, Pilote L. Effects of early and late administration of angiotensin-converting enzyme inhibitors on mortality after myocardial infarction. Am J Med 2003;115:473-479

48. White HD. Should all patients with coronary disease receive angiotensin-converting-enzyme inhibitors? Lancet 2003;362:755-757

49. Officers A, Coordinators for the ACRGTA, Lipid-Lowering Treatment to Prevent Heart Attack T. Major outcomes in high-risk hypertensive patients randomized to angiotensin-converting enzyme inhibitor or calcium channel blocker vs diuretic: The Antihypertensive and Lipid-Lowering Treatment to Prevent Heart Attack Trial (ALLHAT). JAMA 2002;288:2981-2997

50. Turnbull F, Blood Pressure Lowering Treatment Trialists C. Effects of different blood-pressure-lowering regimens on major cardiovascular events: results of prospectively-designed overviews of randomised trials. Lancet 2003;362:1527-1535

51. Brunzell JD, Davidson M, Furberg CD, et al. Lipoprotein management in patients with cardiometabolic risk: consensus conference report from the American Diabetes Association and the American College of Cardiology Foundation. J Am Coll Cardiol 2008;51:1512-1524

52. Levy AR, Briggs AH, Demers C, et al. Cost-effectiveness of beta-blocker therapy with metoprolol or with carvedilol for treatment of heart failure in Canada. Am Heart J 2001;142:537-543

53. Chowdhury EK, Ademi Z, Moss JR, et al. Cost-utility of angiotensin-converting enzyme inhibitor-based treatment compared with thiazide diuretic-based treatment for hypertension in elderly Australians considering diabetes as comorbidity. Medicine (Baltimore) 2015;94:e590

54. Boyd CM, Leff B, Wolff JL, et al. Informing clinical practice guideline development and implementation: prevalence of coexisting conditions among adults with coronary heart disease. J Am Geriatr Soc 2011;59:797805

55. Sachdev M, Sun JL, Tsiatis AA, et al. The prognostic importance of comorbidity for mortality in patients with stable coronary artery disease. J Am Coll Cardiol 2004;43:576-582

56. Koene RJ, Prizment AE, Blaes A, et al. Shared Risk Factors in Cardiovascular Disease and Cancer. Circulation 2016;133:1104-1114

57. Thomas JA, 2nd, Gerber L, Banez LL, et al. Prostate cancer risk in men with baseline history of coronary artery disease: results from the REDUCE Study. Cancer Epidemiol Biomarkers Prev 2012;21:576-581

58. Neugut AI, Rosenberg DJ, Ahsan H, et al. Association between coronary heart disease and cancers of the breast, prostate, and colon. Cancer Epidemiol Biomarkers Prev 1998;7:869-873

59. Chan AO, Jim MH, Lam KF, et al. Prevalence of colorectal neoplasm among patients with newly diagnosed coronary artery disease. JAMA 2007;298:1412-1419

60. Yusuf SW, Razeghi P, Yeh ET. The diagnosis and management of cardiovascular disease in cancer patients. Curr Probl Cardiol 2008;33:163-196 
61. Herrmann J, Lerman A, Sandhu NP, et al. Evaluation and management of patients with heart disease and cancer: cardio-oncology. Mayo Clin Proc 2014;89:1287-1306

62. Accordino MK, Neugut AI, Hershman DL. Cardiac effects of anticancer therapy in the elderly. J Clin Oncol 2014;32:2654-2661

63. Marks LB, Constine, L.S., Adams, M.J., . Cardiotoxicity of radiation therapy for malignancy. 2015. Available at: http://www.uptodate.com/contents/cardiotoxicity-of-radiation-therapy-for-malignancy. Accessed December 30, 2015

64. Floyd J, Morgan, J.P., Berg, S.,. Cardiotoxicity of anthracycline-like chemotherapy agents. 2015. Available at: http://www.uptodate.com/contents/cardiotoxicity-of-anthracycline-like-chemotherapy-agents. Accessed December 30, 2015

65. Morgan JP. Cardiotoxicity of trastazumab and other HER2-targeted agents. 2015. Available at: http://www.uptodate.com/contents/cardiotoxicity-of-trastuzumab-and-other-her2-targeted-agents. Accessed December 30, 2015

66. Smith MR, Crawford, E.D.,. Side effects of androgen deprivation therapy. 2015. Available at: http://www.uptodate.com/contents/side-effects-of-androgen-deprivation-therapy. Accessed December 30, 2015

67. Floyd J, Morgan, J.P.,. Cardiotoxicity of nonanthracycline cancer chemotherapy agents. 2015. Available at: http://www.uptodate.com/contents/cardiotoxicity-of-nonanthracycline-cancer-chemotherapy-agents. Accessed December 30, 2015

68. Kung HC, Hoyert DL, Xu J, et al. Deaths: final data for 2005. Natl Vital Stat Rep 2008;56:1-120

69. Piette JD, Kerr EA. The impact of comorbid chronic conditions on diabetes care. Diabetes Care 2006;29:725731

70. Bradshaw PT, Stevens J, Khankari N, et al. Cardiovascular Disease Mortality Among Breast Cancer Survivors. Epidemiology 2016;27:6-13

71. Kalam K, Marwick TH. Role of cardioprotective therapy for prevention of cardiotoxicity with chemotherapy: a systematic review and meta-analysis. Eur J Cancer 2013;49:2900-2909

72. Krone RJ. Managing coronary artery disease in the cancer patient. Prog Cardiovasc Dis 2010;53:149-156

73. Quality AfHRa. Optimizing Prevention and Healthcare Management for the Complex Patient. 2007. Available at: http://archive.ahrq.gov/fund/trans101507.htm. Accessed May 27, 2016

74. Soni A. Top 10 Most Costly Conditions among Men and Women, 2008: Estimates for the U.S. Civilian Noninstitutionalized Adult Population, Age 18 and Older, Statistical Brief \#331. Rockville, MD2011

75. Olin GL, and Rhoades, J. A.,. The Five Most Costly Medical Conditions, 1997 and 2002: Estimates for the U.S. Civilian Noninstitutionalized Population, Statistical Brief \#80. . Rockville, MD2005

76. Mariotto AB, Yabroff KR, Shao Y, et al. Projections of the cost of cancer care in the United States: 2010-2020. J Natl Cancer Inst 2011;103:117-128

77. National Institutes of Health. Morbidity \& mortality: 2012 chartbook on cardiovascular, lung, and blood diseases. 2012

78. Kannel WB. Coronary heart disease risk factors in the elderly. Am J Geriatr Cardiol 2002;11:101-107 
79. Elveback LR, Connolly DC. Coronary heart disease in residents of Rochester, Minnesota. V. Prognosis of patients with coronary heart disease based on initial manifestation. Mayo Clin Proc 1985;60:305-311

80. Kannel WB, Feinleib M. Natural history of angina pectoris in the Framingham study. Prognosis and survival. Am J Cardiol 1972;29:154-163

81. Hodgson TA, Cohen AJ. Medical care expenditures for selected circulatory diseases: opportunities for reducing national health expenditures. Med Care 1999;37:994-1012

82. Yazdanyar A, Newman AB. The burden of cardiovascular disease in the elderly: morbidity, mortality, and costs. Clin Geriatr Med 2009;25:563-577, vii

83. Newman AB, Gottdiener JS, McBurnie MA, et al. Associations of subclinical cardiovascular disease with frailty. J Gerontol A Biol Sci Med Sci 2001;56:M158-166

84. Newman AB, Arnold AM, Naydeck BL, et al. "Successful aging": effect of subclinical cardiovascular disease. Arch Intern Med 2003;163:2315-2322

85. de Rijke JM, Schouten LJ, Hillen HF, et al. Cancer in the very elderly Dutch population. Cancer 2000;89:11211133

86. Lloyd-Jones DM, Larson MG, Beiser A, et al. Lifetime risk of developing coronary heart disease. Lancet 1999;353:89-92

87. Seshadri S, Beiser A, Kelly-Hayes M, et al. The lifetime risk of stroke: estimates from the Framingham Study. Stroke 2006;37:345-350

88. Bairey Merz CN, Shaw LJ, Reis SE, et al. Insights from the NHLBI-Sponsored Women's Ischemia Syndrome Evaluation (WISE) Study: Part II: gender differences in presentation, diagnosis, and outcome with regard to gender-based pathophysiology of atherosclerosis and macrovascular and microvascular coronary disease. J Am Coll Cardiol 2006;47:S21-29

89. Kreatsoulas C, Anand SS, Subramanian SV. An emerging double burden of disease: the prevalence of individuals with cardiovascular disease and cancer. J Intern Med 2014;275:494-505

90. Coebergh JW, Janssen-Heijnen ML, Post PN, et al. Serious co-morbidity among unselected cancer patients newly diagnosed in the southeastern part of The Netherlands in 1993-1996. J Clin Epidemiol 1999;52:11311136

91. Bauters C, Deneve M, Tricot O, et al. Prognosis of patients with stable coronary artery disease (from the CORONOR study). Am J Cardiol 2014;113:1142-1145

92. van Domburg RT, Kappetein AP, Bogers AJ. The clinical outcome after coronary bypass surgery: a 30-year follow-up study. Eur Heart J 2009;30:453-458

93. Osterberg L, Blaschke T. Adherence to medication. N Engl J Med 2005;353:487-497

94. Bitton A, Choudhry NK, Matlin OS, et al. The impact of medication adherence on coronary artery disease costs and outcomes: a systematic review. Am J Med 2013;126:357.e357-357.e327

95. Chowdhury R, Khan H, Heydon E, et al. Adherence to cardiovascular therapy: a meta-analysis of prevalence and clinical consequences. Eur Heart J 2013;34:2940-2948

96. Wald NJ, Law MR. A strategy to reduce cardiovascular disease by more than 80\%. BMJ 2003;326:1419 
97. Centers for Medicare and Medicaid Services. Chronic Conditions among Medicare Beneficiaries, Chartbook, 2012 ed. 2012. Available at: http://www.cms.gov/Research-Statistics-Data-and-Systems/Statistics-Trends-andReports/Chronic-Conditions/Downloads/2012Chartbook.pdf. Accessed March 9, 2016

98. National Council on Aging. Healthy Aging Factsheet. Arlington, VA2012

99. Kaiser Family Foundation. Distribution of Medicare Beneficiaries by Eligibility Category. 2016. Available at: http://kff.org/medicare/state-indicator/distribution-of-medicare-beneficiaries-by-eligibility-category-2/. Accessed May 27, 2016

100. Centers for Medicare and Medicaid Services. Medicare \& Medicaid Statistical Supplement. 2006. Available at: https://www.cms.gov/Research-Statistics-Data-and-Systems/Statistics-Trends-andReports/MedicareMedicaidStatSupp/index.html?redirect=/MedicareMedicaidStatSupp/. Accessed March 4, 2016

101. Potetz L, DeWilde, LF. Cancer and Medicare - A chartbook. 2009

102. Berger NA, Savvides P, Koroukian SM, et al. Cancer in the elderly. Trans Am Clin Climatol Assoc 2006;117:147-155; discussion 155-146

103. Centers for Medicare \& Medicaid Services. Chronic Conditions. 2015. Available at: http://www.cms.gov/Research-Statistics-Data-and-Systems/Statistics-Trends-and-Reports/ChronicConditions/index.html Accessed May 16, 2016

104. McDevitt RD. A Medicare Buy-In: Examining the Costs for Two Populations. 1998

105. United States Cancer Statistics: 1999-2012 Incidence and Mortality Web-based Report. 2014. Available at: https://nccd.cdc.gov/uscs/. Accessed April 3, 2015

106. American Cancer Society. Cancer facts \& figures 2016. In: Society AC, ed.2016

107. Surveillance Epidemiology and End Results. Cancer Stat Fact Sheets. 2016. Available at: http://seer.cancer.gov/. Accessed March 18, 2016

108. Chen J, Long JB, Hurria A, et al. Incidence of heart failure or cardiomyopathy after adjuvant trastuzumab therapy for breast cancer. J Am Coll Cardiol 2012;60:2504-2512

109. Doyle JJ, Neugut AI, Jacobson JS, et al. Radiation therapy, cardiac risk factors, and cardiac toxicity in earlystage breast cancer patients. Int J Radiat Oncol Biol Phys 2007;68:82-93

110. Gross CP, McAvay GJ, Guo Z, et al. The impact of chronic illnesses on the use and effectiveness of adjuvant chemotherapy for colon cancer. Cancer 2007;109:2410-2419

111. Hall HI, Satariano WA, Thompson T, et al. Initial treatment for prostate carcinoma in relation to comorbidity and symptoms. Cancer 2002;95:2308-2315

112. Jagsi R, Griffith KA, Koelling T, et al. Stroke rates and risk factors in patients treated with radiation therapy for early-stage breast cancer. J Clin Oncol 2006;24:2779-2785

113. Marr PL, Elkin EP, Arredondo SA, et al. Comorbidity and primary treatment for localized prostate cancer: data from CaPSURE. J Urol 2006;175:1326-1331

114. Otto SJ, Schroder FH, de Koning HJ. Risk of cardiovascular mortality in prostate cancer patients in the Rotterdam randomized screening trial. J Clin Oncol 2006;24:4184-4189 
115. Valderas JM, Starfield B, Sibbald B, et al. Defining comorbidity: implications for understanding health and health services. Ann Fam Med 2009;7:357-363

116. Chen J, Long, J., Chen, C., Wang, S., Steingart, R., Gross, C.P. Many breast cancer patients don't get treatment for heart problems. 2015. Available at: http://newsroom.heart.org/news/many-breast-cancer-patients-dont-gettreatment-for-heart-problems?preview=9880. Accessed April 2, 2015

117. Snyder CF, Frick KD, Herbert RJ, et al. Quality of care for comorbid conditions during the transition to survivorship: differences between cancer survivors and noncancer controls. J Clin Oncol 2013;31:1140-1148

118. investigators. Cr. Prospective observational longitudinal registry of patients with stable coronary artery disease. 2016. Available at: http://www.clarify-registry.com/. Accessed May 27, 2016

119. Ortman JM, Velkoff, V.A., Hogan, H. An Aging Nation: The Older Population in the United States. 2014

120. Townsley CA, Selby R, Siu LL. Systematic review of barriers to the recruitment of older patients with cancer onto clinical trials. J Clin Oncol 2005;23:3112-3124

121. Gellad WF, Grenard, J., McGlynn, E.A. A Review of Barriers to Medication Adherence: A Framework for Driving Policy Options. 2009. Available at: http://www.rand.org/pubs/technical_reports/TR765.html. Accessed May 27, 2016

122. Murray MD, Morrow DG, Weiner M, et al. A conceptual framework to study medication adherence in older adults. Am J Geriatr Pharmacother 2004;2:36-43

123. Unni E, Farris KB. Determinants of different types of medication non-adherence in cholesterol lowering and asthma maintenance medications: a theoretical approach. Patient Educ Couns 2011;83:382-390

124. Andersen RM. Revisiting the behavioral model and access to medical care: does it matter? J Health Soc Behav 1995;36:1-10

125. Andersen R, Newman JF. Societal and individual determinants of medical care utilization in the United States. Milbank Mem Fund Q Health Soc 1973;51:95-124

126. Engels EA, Pfeiffer RM, Ricker W, et al. Use of surveillance, epidemiology, and end results-medicare data to conduct case-control studies of cancer among the US elderly. Am J Epidemiol 2011;174:860-870

127. Warren JL, Klabunde CN, Schrag D, et al. Overview of the SEER-Medicare data: content, research applications, and generalizability to the United States elderly population. Med Care 2002;40:IV-3-18

128. American Community Survey. Summary File Data. 2016. Available at: https://www.census.gov/programssurveys/acs/data.html. Accessed March 30, 2016

129. Area Health Resource Files (AHRF). 2016. Available at: http://ahrf.hrsa.gov/overview.htm. Accessed March 2, 2016

130. County Health Rankings \& Roadmaps. Rankings data 2016. Available at: http://www.countyhealthrankings.org/rankings/data. Accessed May 30, 2016

131. Ho PM, Magid DJ, Masoudi FA, et al. Adherence to cardioprotective medications and mortality among patients with diabetes and ischemic heart disease. BMC Cardiovasc Disord 2006;6:48

132. McKenzie SJ, McLaughlin D, Clark J, et al. The burden of non-adherence to cardiovascular medications among the aging population in Australia: a meta-analysis. Drugs Aging 2015;32:217-225 
133. De Vera MA, Bhole V, Burns LC, et al. Impact of statin adherence on cardiovascular disease and mortality outcomes: a systematic review. Br J Clin Pharmacol 2014;78:684-698

134. Smith SC, Jr., Benjamin EJ, Bonow RO, et al. AHA/ACCF secondary prevention and risk reduction therapy for patients with coronary and other atherosclerotic vascular disease: 2011 update: a guideline from the American Heart Association and American College of Cardiology Foundation endorsed by the World Heart Federation and the Preventive Cardiovascular Nurses Association. J Am Coll Cardiol 2011;58:2432-2446

135. Jackevicius CA, Mamdani M, Tu JV. Adherence with statin therapy in elderly patients with and without acute coronary syndromes. JAMA 2002;288:462-467

136. Benner JS, Glynn RJ, Mogun H, et al. Long-term persistence in use of statin therapy in elderly patients. JAMA 2002;288:455-461

137. Rajpura J, Nayak R. Medication adherence in a sample of elderly suffering from hypertension: evaluating the influence of illness perceptions, treatment beliefs, and illness burden. J Manag Care Pharm 2014;20:58-65

138. Choudhry NK, Setoguchi S, Levin R, et al. Trends in adherence to secondary prevention medications in elderly post-myocardial infarction patients. Pharmacoepidemiol Drug Saf 2008;17:1189-1196

139. Yap AF, Thirumoorthy T, Kwan YH. Systematic review of the barriers affecting medication adherence in older adults. Geriatr Gerontol Int 2016;16:1093-1101

140. Rashid MA, Edwards D, Walter FM, et al. Medication taking in coronary artery disease: a systematic review and qualitative synthesis. Ann Fam Med 2014;12:224-232

141. Ho PM, Bryson CL, Rumsfeld JS. Medication adherence: its importance in cardiovascular outcomes. Circulation 2009;119:3028-3035

142. Yap AF, Thirumoorthy, T., Kwan, Y.H. Medication adherence in the elderly. Journal of Clinical Gerontology \& Geriatrics 2016;7:64-67

143. Ahern TP, Pedersen L, Tarp M, et al. Statin prescriptions and breast cancer recurrence risk: a Danish nationwide prospective cohort study. J Natl Cancer Inst 2011;103:1461-1468

144. Cardwell CR, Hicks BM, Hughes C, et al. Statin use after colorectal cancer diagnosis and survival: a population-based cohort study. J Clin Oncol 2014;32:3177-3183

145. Yu O, Eberg M, Benayoun S, et al. Use of statins and the risk of death in patients with prostate cancer. J Clin Oncol 2014;32:5-11

146. Hindler K, Cleeland, C.S., Rivera, E., Collard, C.D. The Role of Statins in Cancer Therapy. The Oncologist 2006;11:306-315

147. Calip GS, Boudreau DM, Loggers ET. Changes in adherence to statins and subsequent lipid profiles during and following breast cancer treatment. Breast Cancer Res Treat 2013;138:225-233

148. Gellad WF, Grenard JL, Marcum ZA. A systematic review of barriers to medication adherence in the elderly: looking beyond cost and regimen complexity. Am J Geriatr Pharmacother 2011;9:11-23

149. Park D.C. JTR. Medication adherence and aging. In: Fisk AD RW, ed. Handbook of Human Factors and the Older Adult San Diego, CA: Academic Press; 1997:257-287 
150. Centers for Medicare \& Medicaid Services. Chronic conditions data warehouse. 2016. Available at: https://www.ccwdata.org/web/guest/condition-categories. Accessed May 18, 2016

151. Nau DP. Proportion of Days Covered (PDC) as a Preferred Method of Measuring Medication Adherence. 2016. Available at: http://www.pqaalliance.org/files/PDCvsMPRfinal.pdf. Accessed March 20, 2016

152. Centers for Medicare \& Medicaid Services. Medicare 2016 Part C \& D Star Rating Technical Notes. 2015. Available at: https://www.cms.gov/Medicare/Prescription-DrugCoverage/PrescriptionDrugCovGenIn/Downloads/2016-Technical-Notes-Preview-1-v2015_08_05.pdf. Accessed October 17, 2016

153. Choudhry NK, Shrank WH, Levin RL, et al. Measuring concurrent adherence to multiple related medications. Am J Manag Care 2009;15:457-464

154. Evans MA. Evaluation of the CMS-HCC Risk Adjustment Model

155. Goodman RA, Posner SF, Huang ES, et al. Defining and measuring chronic conditions: imperatives for research, policy, program, and practice. Prev Chronic Dis 2013;10:E66

156. Medicare. Drug coverage (Part D). 2016. Available at: https://www.medicare.gov/part-d/index.html. Accessed July 10, 2016

157. Szmaragd C, Clarke, P., Steele, F.,. Subject specific and population average models for binary longitudinal data: a tutorial. Longitudinal and Life Course Studies 2013;4:147-165

158. Hanley JA, Negassa A, Edwardes MD, et al. Statistical analysis of correlated data using generalized estimating equations: an orientation. Am J Epidemiol 2003;157:364-375

159. Chapman RH, Yeaw J, Roberts CS. Association between adherence to calcium-channel blocker and statin medications and likelihood of cardiovascular events among US managed care enrollees. BMC Cardiovasc Disord 2010;10:29

160. Baroletti S, Dell'Orfano H. Medication adherence in cardiovascular disease. Circulation 2010;121:1455-1458

161. Centers for Medicare \& Medicaid Services. Proposed Policy, Payment, and Quality Provisions Changes to the Medicare Physician Fee Schedule for Calendar Year (CY) 2017. 2016. Available at:

https://www.cms.gov/Newsroom/MediaReleaseDatabase/Fact-sheets/2016-Fact-sheets-items/2016-07-072.html. Accessed November 1, 2016

162. Williams A, Manias E, Walker R. Interventions to improve medication adherence in people with multiple chronic conditions: a systematic review. J Adv Nurs 2008;63:132-143

163. Wittayanukorn S, Westrick SC, Hansen RA, et al. Evaluation of medication therapy management services for patients with cardiovascular disease in a self-insured employer health plan. J Manag Care Pharm 2013;19:385395

164. Ahern TP, Lash TL, Thwin SS, et al. Impact of acquired comorbidities on all-cause mortality rates among older breast cancer survivors. Med Care 2009;47:73-79

165. Viswanathan M, Golin CE, Jones CD, et al. Closing the quality gap: revisiting the state of the science (vol. 4: medication adherence interventions: comparative effectiveness). Evid Rep Technol Assess (Full Rep) 2012:1685 
166. Beadles CA, Farley JF, Ellis AR, et al. Do medical homes increase medication adherence for persons with multiple chronic conditions? Med Care 2015;53:168-176

167. Levant S, Owings, M., DeFrances, C. Rate of Hospitalization for Coronary Heart Disease, by Age Group — National Hospital Discharge Survey, United States, 2000-2010. 2012. Available at:

http://www.cdc.gov/mmwr/preview/mmwrhtml/mm6123a5.htm. Accessed November 20, 2016

168. HCUPnet. Statistics on hospital stays. 2010. Available at: http://hcupnet.ahrq.gov/. Accessed Novenber 23, 2016

169. Russell MW, Huse DM, Drowns S, et al. Direct medical costs of coronary artery disease in the United States. Am J Cardiol 1998;81:1110-1115

170. Raval AD, Madhavan S, Mattes MD, et al. Impact of Prostate Cancer Diagnosis on Noncancer Hospitalizations Among Elderly Medicare Beneficiaries With Incident Prostate Cancer. J Natl Compr Canc Netw 2016;14:186194

171. Zoller B, Ji J, Sundquist J, et al. Risk of coronary heart disease in patients with cancer: a nationwide follow-up study from Sweden. Eur J Cancer 2012;48:121-128

172. Darby SC, Ewertz M, McGale P, et al. Risk of ischemic heart disease in women after radiotherapy for breast cancer. N Engl J Med 2013;368:987-998

173. McGale P, Darby SC, Hall P, et al. Incidence of heart disease in 35,000 women treated with radiotherapy for breast cancer in Denmark and Sweden. Radiother Oncol 2011;100:167-175

174. Onwudiwe NC, Kwok Y, Onukwugha E, et al. Cardiovascular event-free survival after adjuvant radiation therapy in breast cancer patients stratified by cardiovascular risk. Cancer medicine 2014;3:1342-1352

175. Yood MU, Wells KE, Alford SH, et al. Cardiovascular outcomes in women with advanced breast cancer exposed to chemotherapy. Pharmacoepidemiol Drug Saf 2012;21:818-827

176. Chavez-MacGregor M, Zhang N, Buchholz TA, et al. Trastuzumab-related cardiotoxicity among older patients with breast cancer. J Clin Oncol 2013;31:4222-4228

177. Van Hemelrijck M, Garmo H, Holmberg L, et al. Absolute and relative risk of cardiovascular disease in men with prostate cancer: results from the Population-Based PCBaSe Sweden. J Clin Oncol 2010;28:3448-3456

178. Robinson D, Garmo H, Lindahl B, et al. Ischemic heart disease and stroke before and during endocrine treatment for prostate cancer in PCBaSe Sweden. Int J Cancer 2012;130:478-487

179. Keating NL, Zaslavsky AM, Herrinton LJ, et al. Quality of diabetes care among cancer survivors with diabetes. Med Care 2007;45:869-875

180. Greer SA, Nwaise, I.A., Casper, M.L.,. 2010 Atlas of Heart Disease Hospitalizations Among Medicare Beneficiaries. Atlanta, U.S.: Department of Health and Human Services; 2010

181. Cancer AJCo. AJCC Cancer Staging Manual, Sixth edition. Chicago, IL: Springer; 2002

182. Hubbard AE, Ahern J, Fleischer NL, et al. To GEE or not to GEE: comparing population average and mixed models for estimating the associations between neighborhood risk factors and health. Epidemiology 2010;21:467-474 
183. Mayo Clinic. Cardio-oncology clinics integrate specialty clinical care. Available at: http://www.mayoclinic.org/medical-professionals/clinical-updates/cardiovascular/cardio-oncology-clinicsintegrate-specialty-clinical-care. Accessed November 22, 2016

184. Mark TL, Tomic KS, Kowlessar N, et al. Hospital readmission among medicaid patients with an index hospitalization for mental and/or substance use disorder. J Behav Health Serv Res 2013;40:207-221

185. Carroll AE. The Power of Simple Life Changes to Prevent Heart Disease. 2016. Available at: http://www.nytimes.com/2016/12/12/upshot/the-power-of-simple-life-changes-to-prevent-heartdisease.html?contentCollection=smarter-living\&hp\&action=click\&pgtype=Homepage\&clickSource=storyheading\&module=second-column-region\&_r=0. Accessed December 22, 2016

186. Dieleman JL, Baral R, Birger M, et al. US Spending on Personal Health Care and Public Health, 1996-2013. JAMA 2016;316:2627-2646

187. Druss BG, Marcus SC, Olfson M, et al. Comparing the national economic burden of five chronic conditions. Health Aff (Millwood) 2001;20:233-241

188. Tarride JE, Lim M, DesMeules M, et al. A review of the cost of cardiovascular disease. Can J Cardiol 2009;25:e195-202

189. Gerteis J, Izrael D, Deitz D, et al. Multiple Chronic Conditions Chartbook: 2010 Medical Expenditure Panel Survey Data. 2014. Accessed January 23, 2017

190. Fillenbaum GG, Pieper CF, Cohen HJ, et al. Comorbidity of five chronic health conditions in elderly community residents: determinants and impact on mortality. J Gerontol A Biol Sci Med Sci 2000;55:M84-89

191. Nichols GA, Bell TJ, Pedula KL, et al. Medical care costs among patients with established cardiovascular disease. Am J Manag Care 2010;16:e86-e93

192. Wakim R, Ritchey M, Hockenberry J, et al. Geographic Variations in Incremental Costs of Heart Disease Among Medicare Beneficiaries, by Type of Service, 2012. Prev Chronic Dis 2016;13:E180

193. Centers for Medicare \& Medicaid Services. Episode Payment Models: General Information. 2016. Available at: https://innovation.cms.gov/initiatives/epm. Accessed January 20, 2017

194. Reform. CfHQP. Fixing the Problems With Attribution, Risk Adjustment, and Episode Groupers. 2016. Available at: http://www.chqpr.org/. Accessed Jan 8, 2017

195. Delivery System Reform, Medicare Payment Reform. 2016. Available at: https://www.cms.gov/Medicare/Quality-Initiatives-Patient-Assessment-Instruments/Value-BasedPrograms/Value-Based-Programs.html. Accessed Jan 6, 2017

196. O'Byrne TJ, Shah ND, Wood D, et al. Episode-based payment: evaluating the impact on chronic conditions. Medicare \& medicaid research review 2013;3

197. Al-Kindi SG, Oliveira GH. Prevalence of Preexisting Cardiovascular Disease in Patients With Different Types of Cancer: The Unmet Need for Onco-Cardiology. Mayo Clin Proc 2016;91:81-83

198. Whellan DJ, Greiner MA, Schulman KA, et al. Costs of inpatient care among Medicare beneficiaries with heart failure, 2001 to 2004. Circ Cardiovasc Qual Outcomes 2010;3:33-40 
199. Hall AE, Highfill, T.,. Calculating Disease-Based Medical Care Expenditure Indexes for Medicare Beneficiaries: A Comparison of Method and Data Choices. 2014. Available at: http://www.nber.org/papers/w19720. Accessed June 17, 2016

200. Centers for Medicare \& Medicaid Services. Outpatient hospital services. 2015. Available at: https://www.medicare.gov/coverage/outpatient-hospital-services.html. Accessed February 1, 2017

201. National Cancer Institute. SEER-Medicare: Medicare Claims Files. 2016. Available at: https:/healthcaredelivery.cancer.gov/seermedicare/medicare/claims.html?\&url=/seermedicare/medicare/claims .html\#carrier. Accessed February 2, 2017

202. Statistics BoL. Consumer Price Index. 2017. Available at: https://www.bls.gov/cpi/. Accessed January 24, 2017

203. Huxley RR, Woodward M. Cigarette smoking as a risk factor for coronary heart disease in women compared with men: a systematic review and meta-analysis of prospective cohort studies. Lancet 2011;378:1297-1305

204. Djousse L, Gaziano JM. Alcohol consumption and heart failure: a systematic review. Curr Atheroscler Rep 2008;10:117-120

205. Baser O. Modeling Healthcare Costs. STATinMED Research; 2012

206. Pagano E, Petrelli A, Picariello R, et al. Is the choice of the statistical model relevant in the cost estimation of patients with chronic diseases? An empirical approach by the Piedmont Diabetes Registry. BMC Health Serv Res 2015;15:582

207. Mihaylova B, Briggs A, O'Hagan A, et al. Review of statistical methods for analysing healthcare resources and costs. Health Econ 2011;20:897-916

208. Centers for Medicare \& Medicaid Services. Shared Savings Program. 2017. Available at: https://www.cms.gov/Medicare/Medicare-Fee-for-ServicePayment/sharedsavingsprogram/index.html?redirect=/SharedSavingsProgram/. Accessed February 4, 2017

209. Hussey PS, Ridgely MS, Rosenthal MB. The PROMETHEUS bundled payment experiment: slow start shows problems in implementing new payment models. Health Aff (Millwood) 2011;30:2116-2124

210. Centers for Medicare \& Medicaid Services. Readmissions Reduction Program (HRRP). 2016. Available at: https://www.cms.gov/medicare/medicare-fee-for-service-payment/acuteinpatientpps/readmissions-reductionprogram.html. Accessed February 4, 2017

211. Neilsen M, Beult L, Patel K, et al. The Patient-Centered Medical Home’s Impact on Cost and Quality. 2016. Available at: https://www.pcpcc.org/sites/default/files/resources/The\%20PatientCentered\%20Medical\%20Home\%27s\%20Impact\%20on\%20Cost\%20and\%20Quality\%2C\%20Annual\%20Rev iew\%20of\%20Evidence\%2C\%202014-2015.pdf. Accessed February 4, 2017

212. Maeng DD, Khan N, Tomcavage J, et al. Reduced acute inpatient care was largest savings component of Geisinger Health System's patient-centered medical home. Health Aff (Millwood) 2015;34:636-644

213. Chiha J, Mitchell P, Gopinath B, et al. Gender differences in the severity and extent of coronary artery disease. IJC Heart \& Vasculature 2015;8:161-166 


\section{Appendix A. Codes related to cancer diagnosis and treatment}

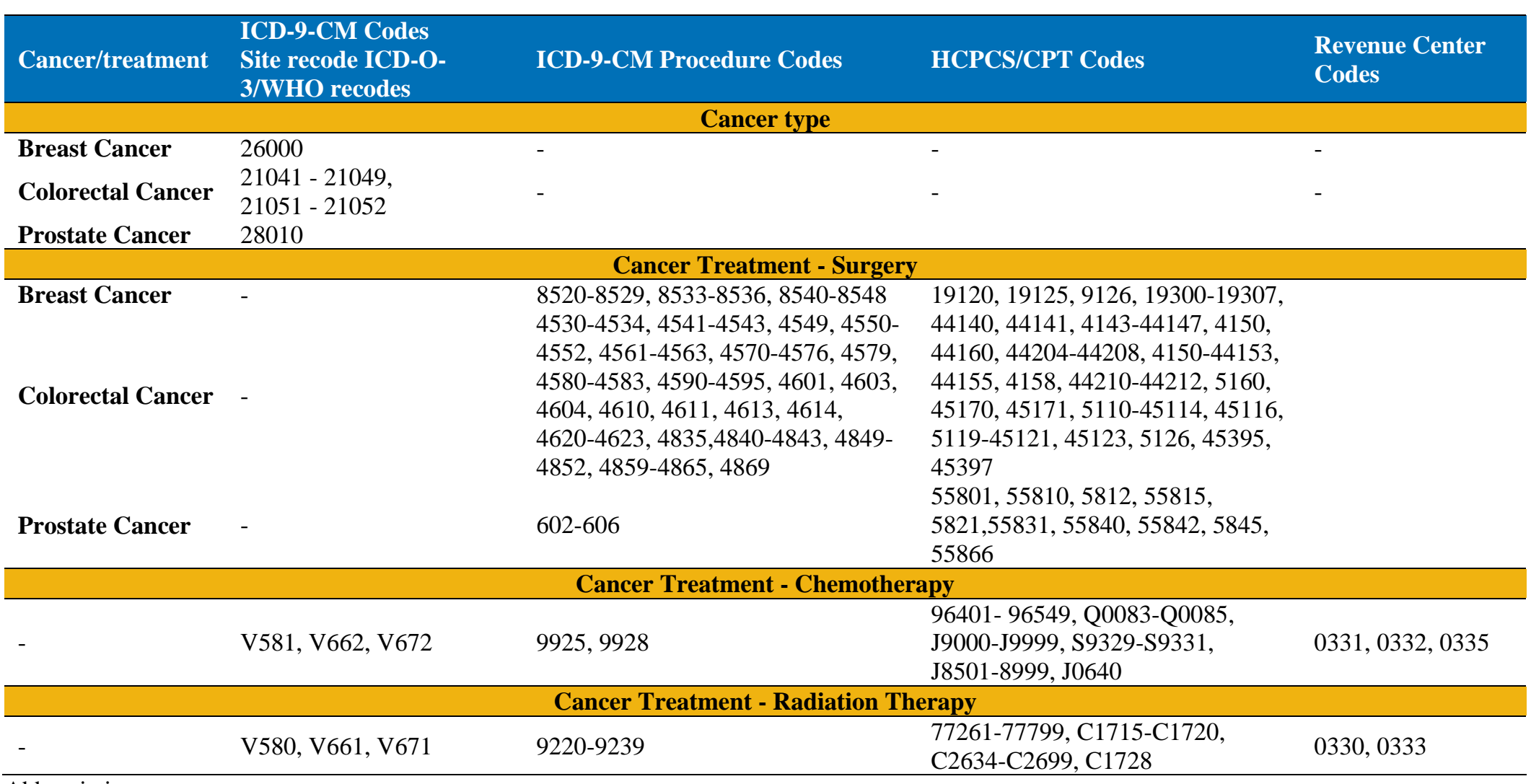

Abbreviations:

CPT-4: Common Procedural Terminology, 4th Edition; HCPCS: Health Care Procedure Classification Code; ICD-9-CM: International Classification of

Diseases, Ninth Revision, Clinical Modification; ICD-O-3: International Classification of Diseases for Oncology, $3^{\text {rd }}$ Edition; WHO: World Health Organization 


\section{Appendix B. List of statins}

\begin{tabular}{|ll|ll|}
\hline \multicolumn{2}{|c|}{ Monotherapy } & \multicolumn{2}{c|}{ Combination therapy } \\
\hline Brand & Generic & Brand & Generic \\
\hline Lipitor & Atorvastatin & Advicor & Lovastatin-Niacin \\
Lescol & Fluvastatin & Caudet & Atorvastatin-Amlodipine \\
Altocor, Mevacor & Lovastatin & Inegy & Simvastatin-Ezetimibe \\
Livalo & Pitavastatin & Simcor & Simvastatin-Niacin \\
Pravachol & Pravastatin & Juvisync & Simvastatin-Sitagliptin \\
Crestor & Rosuvastatin & Liptruzet & Ezetimibe/Atorvastatin calcium \\
Zocor & Simvastatin & & \\
\hline
\end{tabular}




\section{Appendix C. List of angiotensin-converting enzyme inhibitors}

\begin{tabular}{|ll|ll|}
\hline \multicolumn{2}{|c|}{ Monotherapy } & \multicolumn{2}{c|}{ Combination therapy } \\
\hline Brand & Generic & Brand & Generic \\
\hline Lotensin & Benazepril & Accuretic & Quinapril-Hydrochlorothiazide \\
Capoten & Captopril & Capozide & Captopril-Hydrochlorothiazide \\
Vasotec & Enalapril & Lexxel & Enalapril-Hydrochlorothiazide \\
Monopril & Fosinopril & Lotensin HCT & Benazepril-Hydrochlorothiazide \\
Prinivil, Zestril & Lisinopril & Lotrel & Benazepril-Amlodipdine \\
Univasc & Moexipril & Monopril HCT & Fosinopril-Hydrochlorothiazide \\
Aceon & Perindopril & Prinizide, Zestoretic & Lisinopril-Hydrochlorothiazide \\
Accupril & Quinapril & Tarka & Trandolapril-Verapamil \\
Altace & Ramipril & Uniretic & Moexipril-Hydrochlorothiazide \\
Mavik & Trandolapril & Vasoteretic & Enalapril-Hydrochlorothiazide \\
\hline
\end{tabular}




\section{Appendix D. List of angiotensin II receptor blockers}

\begin{tabular}{|ll|ll|}
\hline \multicolumn{2}{|c|}{ Monotherapy } & \multicolumn{2}{c|}{ Combination therapy } \\
\hline Brand & Generic & Brand & Generic \\
Aiovan & Valsartan & Atacand HCT & Candesartan-Hydrochlorothiazide \\
Tevetan & Candesartan & Tevetan HCT & Eprosartan-Hydrochlorothiazide \\
Avapro & Eprosartan & Avalide & Irbesartan-Hydrochlorothiazide \\
Cozaar & Irbesartan & Hyzaar & Losartan-Hydrochlorothiazide \\
Benicar & Losartan & Benicar HCT & Olmesartan-Hydrochlorothiazide \\
Micardis & Olmesartan & Micardis HCT & Telmisartan-Hydrochlorothiazide \\
Edarbi & Telmisartan & Diovan HCT & Valsartan-Hydrochlorothiazide \\
& Azilsartan & Azor & Olmesartan-Amlodipine \\
& & Exforge & Valsartan-Amlodipine \\
& & Exforge HCT & Valsartan-Amlodipine-Hydrochlorothiazide \\
& & Valturna & Valsartan-Aliskerin \\
& & Edarbyclor & Azilsartan-Chlorthalidone \\
& & Tribenzor & Olmesartan-Amlodipine-Hydrochlorothiazide \\
& & Twynsta & Telmisartan-Amlodipine \\
\hline
\end{tabular}




\section{Appendix E. List of beta-blockers}

\begin{tabular}{|ll|ll|}
\hline \multicolumn{2}{|c|}{ Monotherapy } & \multicolumn{2}{c|}{ Combination therapy } \\
\hline Brand & Generic & Brand & Generic \\
\hline Betapace & Sotalol & Corzide & Nadolol-Bendroflumethiazide \\
Blocadren & Timolol & Inderide & Propanolol-Hydrochlorothiazide \\
Brevibloc & Esmolol & Tenoretic & Atenolol-Chlorthalidone \\
Cartrol & Carteolol & Ziac & Bisoprolol-Hydrochlorothiazide \\
Coreg & Carvedilol & Lopressor-HCT & Metoprolol-Hydrochlorothiazide \\
Corgard & Nadolol & & \\
Inderal & Propanolol & & \\
Kerlone & Betaxolol & & \\
Levatol & Penbutolol & & \\
Lopressor & Metoprolol & & \\
Sectral & Acebutolol & & \\
Tenormin & Atenolol & & \\
Trandate Normodyne & Labetalol & & \\
Visken & Pindolol & & \\
Zebeta & Bisoprolol & & \\
Bystolic & Nebivolol & & \\
\hline
\end{tabular}




\section{Appendix F. Heart disease ICD-9-CM diagnosis and procedure codes}

\begin{tabular}{|l|l|}
\hline \multicolumn{1}{|c|}{ Heart diseases } & \multicolumn{1}{|c|}{ ICD-9-CM diagnosis/procedure code } \\
\hline Acute Myocardial infarction & $410.01,410.11,410.21,410.31,410.41,410.51,410.61,410.71,410.81,410.91$ \\
\hline Ischemic Heart Disease & $410.00,410.01,410.02,410.10,410.11,410.12,410.20,410.21,410.22,410.30$, \\
& $410.31,410.32,410.40,410.41,410.42,410.50,410.51,410.52,410.60,410.61$, \\
& $410.62,410.70,410.71,410.72,410.80,410.81,410.82,410.90,410.91,410.92$, \\
& $411.0,411.1,411.81,411.89,412,413.0,413.1,413.9,414.00,414.01,414.02$, \\
& $414.03,414.04,414.05,414.06,414.07,414.12,414.2,414.3,414.4,414.8,414.9$ \\
\hline Stroke / Transient Ischemic & $430,431,433.01,433.11,433.21,433.31,433.81,433.91,434.00,434.01,434.10$, \\
Attack & $434.11,434.90,434.91,435.0,435.1,435.3,435.8,435.9,436,997.02$ \\
\hline Heart Failure & $398.91,402.01,402.11,402.91,404.01,404.03,404.11,404.13,404.91,404.93$, \\
& $428.0,428.1,428.20,428.21,428.22,428.23,428.30,428.31,428.32,428.33$, \\
\hline CAD-related Procedures & $428.40,428.41,428.42,428.43,428.9$ \\
\hline & $0066,3601,3602,3603,3604,3605,3606,3607,3609,3610,3611,3612$, \\
\hline
\end{tabular}

Note: Other heart diseases (stroke and heart failure) and procedure codes were used for CAD-related hospitalization. 